\title{
Ergebnisse von Grossversuchen an hochbelasteten Belebtschlammanlagen und Tropfkörpern
}

\author{
Von K. WUHRMANN
}

(Mitteilung aus der Eidgenössischen Anstalt für Wasserversorgung, Abwasserreinigung und Gewässerschutz an der Eidgenössischen Technischen Hochschule in Zürich)

Eingegangen am 16. März 1953핀

\section{INHALTSVERZEICHNIS}

Vorbemerkung ...................... 2

Einleitung ...................... . . 3

I. Teil: Grundlagen der Versuche

A. Problemstellung . . . . . . . . . . . . . . . . . . . 4

B. Versuchsanlagen .. . . . . . . . . . . . . . . . . 6

I. Belebtschlammanlage . . . . . . . . . . . . . . . . . 7

2. Künstlich belüfteter Tropfkörper . . . . . . . . . . . . . . . II

C. Betrieb der Versuchsanlagen . . . . . . . . . . . . . . . . . . . . I4

I. Rohwasser. . . . . . . . . . . . . . . . . . . . . I4

2. Durchfuihrung der Versuche . . . . . . . . . . . . I9

3. Probenahme ................. . . 20

a) Sammelproben während $24 \mathrm{~h} \ldots . . . .20$

b) Einzelproben . . . . . . . . . . . . . . . . . 2 2 I

c) Voraussetzung für die Vergleichbarkeit von Proben . . . . . . . . 22

4. Untersuchungsmethoden . . . . . . . . . . . . . . . 24

a) Betriebstechnische Untersuchungen ............ 25

b) Prüfung der Reinigungsleistung einer Anlage . . . . . . . . 25

II. Teil: Durchgefübrte Versucbe, Betriebsbeobacbtungen und Analysenergebrisse

A. Versuche mit dem Belebtschlammverfahren . . . . . . . . . . . 27

B. Versuche mit dem künstlich belüfteten Tropfkörper . . . . . . . . . . 28

C. Bemerkungen zu den Tabellen 9 bis 17. . . . . . . . . . . . . . . . 48

\section{Teil: Ausmertung der Beobacbtungen}

A. Bemerkungen zum Betriebe der Versuchsanlagen und den Verfahren im allgemeinen . . . . . . . . . . . . . . . . . 49

r. Beobachtungen beim Belebtschlammverfahren . . . . . . . . . . . 49

a) Belüftung und Schlammkonzentration im Belüftungsbecken . . . . 49

b) Wartung der Belebtschlammanlage . . . . . . . . . . 56

1) Manuskript abgeschlossen im Oktober 1952. 
c) Bürstenbelüftung . . . . . . . . . . . . . . . . . 58

d) Zusätze zum Belebtschlamm . . . . . . . . . . . . . . . . 60

2. Beobachtungen am künstlich belüfteten Tropfkörper . . . . . . . . 6 I

B. Entfernung von Stoffen mit biochemischem Sauerstoff bedarf . . . . . . 62

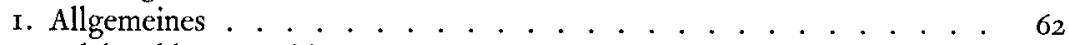

2. Belebtschlammverfahren............... . 64

3. Künstlich belüfteter Tropf körper . . . . . . . . . . . 7I

C. Umsatz der Stickstoffverbindungen . . . . . . . . . . . . . . . 76

x. Umwandlung der Stickstoffverbindungen . . . . . . . . . . . 76

2. Elimination des Stickstoffs . . . . . . . . . . . . . . . . 79

3. Stickstoffbilanz der Reinigungsanlagen . . . . . . . . . . . . 8 r

D. Phosphorelimination . . . . . . . . . . . . . . . . . 83

E. Schlammproduktion und Schlammzusammensetzung . . . . . . . . 85

I. Überschußschlammengen ... . . . . . . . . . . . 85

2. Schlammzusammensetzung . . . . . . . . . . . 88

IV. Teil: Scblussbemerkungen und Zusammenfassung

Zusammenfassung . . . . . . . . . . . . . . . . 9I

Literaturverzeichnis . . . . . . . . . . . . . . . . 92

\section{VORBEMERKUNG}

Die nachfolgende Übersicht enthält die Messungen und Beobachtungen, welche die Eidgenössische Anstalt für Wasserversorgung, Abwasserreinigung und Gewässerschutz in den letzten Jahren auf den Versuchsanlagen des Instituts bei der biologischen Reinigung vorgeklärten städtischen Abwassers in systematischen Experimenten von technischem Maßstab sammelte. Die Arbeiten wurden anfänglich unter der Leitung der Herren Prof. Dr. E. Meyer-Peter und Prof. Dr. W. von GoNZENBACH, später unter derjenigen von Herrn Dr. U. A. CORTI gemeinsam von der biologischen (K. WUHRMANN, H. WOKER), chemischen (F. ZEHENDER, H. KuISEL) und technischen (A. Kropf, W. Ziegler) Abteilung der Anstalt durchgeführt. Über die einzelnen Versuchsserien ist bisher nichts ausführlich veröffentlicht worden; ihre Ergebnisse wurden nur in internen Berichten niedergelegt oder gelangten in speziellen Rapporten an einzelne Interessenten.

Da die Arbeiten für die uns zunächst am meisten interessierenden Fragestellungen abgeschlossen sind, erfolgt nun eine Zusammenstellung der bisherigen Ergebnisse, die einerseits den gegenwärtigen Stand der Erfahrungen umreissen und gleichzeitig als Basis für die weiteren experimentellen Untersuchungen dienen soll.

An die Erstellung und Erweiterung der Versuchsanlagen wurden im Laufe der Zeit von Kantonen, Gemeinden, der Stiftung zur Förderung schweizerischer Volkswirtschaft durch wissenschaftliche Forschung und vom Jubiläumsfonds der ETH. namhafte Beiträge geleistet. Wir möchten auch an dieser Stelle allen Beteiligten für die Unterstützung unserer Arbeiten bestens danken.

Es liegt uns ferner daran, allen Mitarbeitern der Anstalt in den Laboratorien und auf der Versuchsanlage unseren speziellen Dank für die gewissenhafte Durchführung der umfangreichen Versuchsprogramme auszusprechen. Ihre Arbeit und insbesondere diejenige unseres Mechanikers, Hern Friedrich; der die Versuchsanlagen beaufsichtigte, bildet die Grundlage der nun vorliegenden Ergebnisse. 


\section{EINLEITUNG}

Es ist das Hauptziel dieses Berichtes, die in vielen Versuchen ermittelten Einzelergebnisse unter dem einheitlichen Gesichtspunkt weniger, uns für die praktische Verwirklichung des Gewässerschutzes bedeutsam erscheinender Fragen auszuwerten. Experimente, die aus dem einen oder andern Grund keinen Beitrag an die diskutierten Probleme liefern können, bleiben unerwähnt. Insbesondere konzentriert sich die Darstellung auf die beiden Verfahren: Belebtschlamm und künstlich belüfteter Tropfkörper. Eine Reihe anderer biologischer Reinigungssysteme und Systemkombinationen, die wir gelegentlich in kürzeren Versuchsperioden prüften, werden nicht beschrieben, da die Beobachtungen mindestens vorläufig nicht von praktischem Interesse sind.

Naturgemäss erfolgten unsere Versuche vor allem im Hinblick auf die Abwasserreinigung in Anlagen kleiner oder mittlerer Grösse, wie sie für schweizerische Verhältnisse in erster Linie benötigt werden. Auch die gegenüber bisheriger Praxis relativ hohen Belastungen ergaben sich aus den schweizerischen Gegebenheiten.

Der vorliegende Bericht will nicht nur Rechenschaft über die ausgeführten Arbeiten ablegen, sondern zugleich einige Erfahrungen vermitteln, die einem Projektverfasser oder einer Gemeindebehörde das Vertrauen in die Leistungsfähigkeit und sichere Betriebsweise hochbelasteter Abwasserreinigungsanlagen erwecken sollen.

Die Fragestellungen (vgl. unten) für alle Versuche waren grundsätzlich nur verfabrenstecbnischer und vergleichend-mirtschaftlicber Art. Konstruktipe Probleme wurden nicht - oder nur beiläufig bezüglich Details - studiert, und die einmal errichteten Versuchsanlagen blieben deshalb praktisch unverändert für alle Experimente (vgl. Abschnitt I, a). In bezug auf die technische Gestaltung der Anlagen sind deshalb alle Versuche der Belebtschlamm- und Tropf körpergruppe streng untereinander vergleichbar.

Im Laufe der Zeit wurde auf Grund der Erfahrungen die Versuchstechnik ständig verbessert und vor allem durch präzisere Fragestellungen und eine entsprechende Untersuchungsmethodik die Ausbeute der Experimente vergrössert. Dementsprechend besitzen die in den letzten sechs Jahren ausgeführten Versuchsserien im Hinblick auf das Ziel dieses Berichtes wesentlich grössere Bedeutung als die früheren. Die mit den ersten Versuchen geleistete Vorarbeit war natürlich unentbehrlich für die später erzielten Ergebnisse. 
Der vorliegende Bericht enthält im ersten Teil eine kurze Darstellung der Grundlagen, welche als Ausgangspunkt für die Versuche und die speziellen Fragestellungen dienten. Ferner ist dort die Versuchstechnik beschrieben. Im II. Teil geben wir an Hand tabellarischer Zusammenstellungen die ermittelten Beobachtungen in den einzelnen Experimenten (soweit sie für diesen Bericht berücksichtigt werden) auszugsweise (Mittelwerte) bekannt. Im III.Teil möchten wir an Hand des Zahlenmaterials einige der zur Zeit wichtigsten Fragen bei der Anwendung der Ergebnisse für die Technik des Gewässerschutzes beantworten.

\section{ERSTER TEIL}

\section{GRUNDLAGEN DER VERSUCHE}

\section{A. Problemstellung}

Als Ausgangspunkt für die Versuche wurde im Jahre I936 die Aufgabe gestellt, eine Abwasserreinigungsmethode zu suchen, die bezüglich ihrer Reinigungswirkung zwischen den Ergebnissen der rein mechanischen Klärung und der "biologischen Vollreinigung» (schwachbelastete Tropfkörper oder Belebtschlammsysteme) liegen sollte. Man sagte sich mit Recht, dass viele Gemeinden in der Schweiz relativ wasserreiche Vorfluter für ihre zukünftigen Abwasseranlagen besitzen und somit eine «Vollreinigung» der Abwässer unnötig sei. Bei der Abwasserreinigung mittels Tropfkörpern war schon vor diesem Zeitpunkt eine Entwicklung $\mathrm{zu}$ hohen Belastungen eingetreten (sogenannte Hochleistungstropfkörper, Tropf körper mit Rezirkulation usw.). Auch bei Belebtschlammanlagen hat man schon seit Bekanntwerden des Verfahrens nach immer höheren Belastungen gestrebt, wenn auch ohne bleibenden Erfolg. So zitiert CHASE (1944) Versuche in Houston (Texas) aus dem Jahre I924, wo mit I h Belüftungszeit ein Reinigungseffekt von $94 \%$ - gemessen am $\mathrm{Bsb}_{5}$ - erreicht worden sein soll. Die von Buswell und Mitarbeitern (I928) veröffentlichten Versuche mit belüfteten Tauchkörperanlagen wurden ebenfalls mit Belüftungszeiten von nur I, $5 \mathrm{~h}$ ausgeführt (gegenüber den damals üblichen Belüftungszeiten von 4 bis $\sim$ Io h). Die Versuche in Birmingham (WHITEHEAD, I925) führten dann allmählich zur Auffassung, dass Belebtschlammanlagen mit hoher Belastung nur bei zusätzlicher Wiederbelüftung ("Reaktivierung») des Rücklaufschlammes möglich seien, eine Behauptung, die bis in die neueste Zeit immer wieder 
geäussert wird (PIPE und FARMER, I937; SIERP, I934; BABBIT, I947; ULLRICH und SMITH, I95I, u.a.m.). Im Auslande erfolgte erstmals eine Abweichung von dieser Auffassung, und die konsequente Durchführung von Versuchen in technischem Maßstab mit hochbelasteten Belebtschlammanlagen durch SETTER (I944). Die Ergebnisse dieser Arbeiten sind aber von unserem Standpunkte aus als wenig befriedigend zu beurteilen (vgl. Abschnitt III, B 2). Laboratoriumsexperimente mit hohen Belastungen (OKUN, 1949; SMITH, I952) zeigten, dass genau wie bei unseren Anlagen, auch ohne Schlamm-«Reaktivierung», ausgezeichnete Resultate zu erwarten sind.

Das Studium hochbelasteter Belebtschlammanlagen wurde uns mit dem Bau einer Versuchsanlage im Jahre 1937 ermöglicht und führte zu den nun vorliegenden Ergebnissen. Der Bereich der seither untersuchten Raumbelastungen im Vergleich mit den im Auslande üblichen Dimensionierungsgrundlagen von Belebtschlammanlagen geht am besten aus der Darstellung in Abbildung I hervor, in der wir die in verschiedenen Grossanlagen üblichen hydraulischen Raumbelastungen und Rohwasserkonzentrationen miteinander in Beziehung gesetzt haben, wobei als Unter-

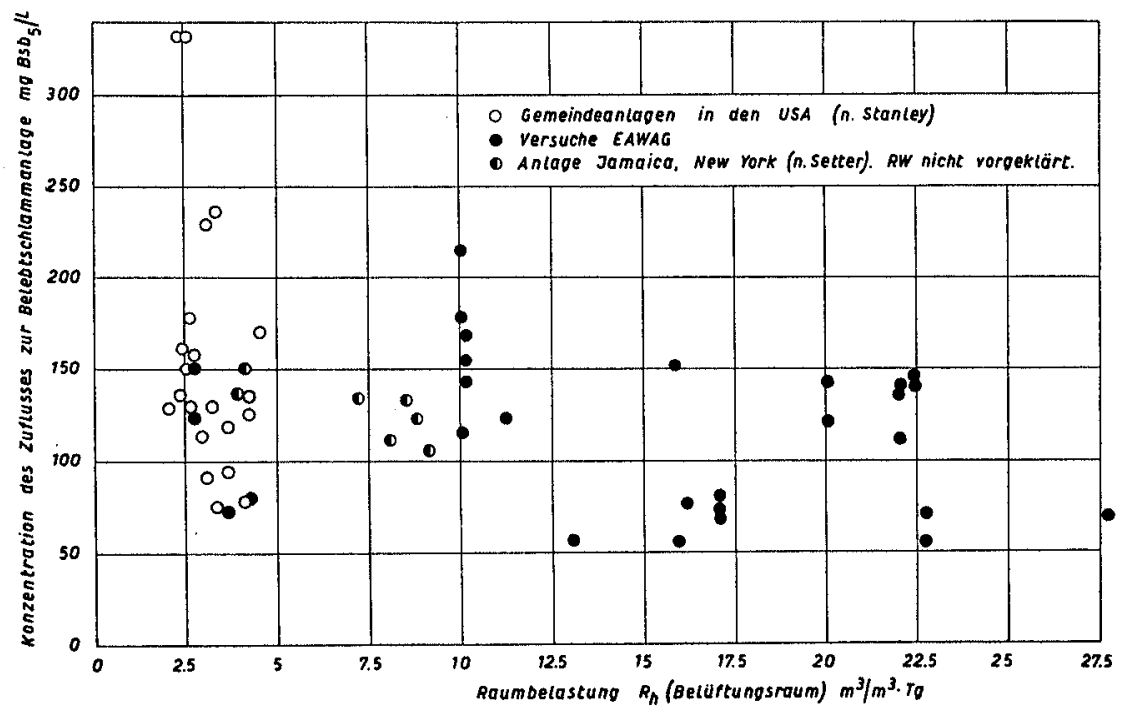

Abb. 1. Untersuchter Belastungsbereich bei den Belebtschlammversuchen im Vergleich mit den Betriebsdaten amerikanischer Abwasserreinigungsanlagen mit Belebtschlammsystem. Ordinate: Konzentration des Zuflusses zu den Belüftungsbecken (mg Bsb $/ 1$ ) im Tagesmittel. Abszisse: Hydraulische Raumbelastung der Belüftungsbecken $\left(\mathrm{m}^{3} / \mathrm{m}^{3} \cdot \mathrm{Tag}\right)$. 
lage die Zusammenstellung von STANLEY (I949) diente. Die Betriebsgrundlagen der Anlage Jamaica in New York wurden besonders hervorgehoben, da sie in der Literatur bereits als typisch für Teilreinigungsanlagen zitiert werden. Aus der Darstellung geht sofort hervor, dass wir unsere Versuche in einem Belastungsgebiet durchführten, das (mindestens in grösseren Anlagen) noch nicht bearbeitet wurde.

Es war selbstverständlich, dass - in Anbetracht der für den Betrieb von Tropfkörpern vielerorts günstigen topographischen Verhältnisse in der Schweiz - auch den hochbelasteten Tropf körpern Aufmerksamkeit geschenkt werden musste. Die Versuchsanlage wurde deshalb $1944 \mathrm{mit}$ der Errichtung von drei Filterbeeten und zugehörigen Nachklärbecken erweitert. Die damit ausgeführten Versuche hielten sich aber in beschränktem Rahmen, und einzig der geschlossene, künstlich belüftete Körper erfuhr eine genauere Prüfung und wird in diesem Bericht berücksichtigt.

Wir können die unseren Experimenten zugrunde liegenden Hauptfragen wie folgt formulieren:

I. Ist es möglich, eine Belebtschlammanlage ohne spezielle Wiederbelüftung des Rücklaufschlammes mit viel kürzerer Belïftungszeit zu betreiben, als dies bis heute üblich ist?

2. Sind die Leistungen einer solchen Anlage für einen wirksamen Schutz der Vorfluter genügend, und welches sind im besonderen die Bedingungen, um eine möglichst hohe Leistung des Systems zu erzielen?

3. In welchem Verhältnis stehen die Leistungen hochbelasteter Belebtschlammanlagen und künstlich belüfteter Tropf körper zueinander, und wo liegt im speziellen die Grenze der Leistungsfähigkeit des letzteren Systems im Hinblick auf eine genügende Qualität des Abflusses?

Neben diesen Grundfragen wurden eine grössere Zahl von Einzelproblemen studiert, die in Abschnitt III dargestellt sind.

\section{B. Versuchsanlagen}

Alle nachstehend beschriebenen Experimente wurden mit den Versuchsanlagen ausgeführt, die das Institut im Laufe der Jahre innerhalb des Areals der Kläranlage Werdhölzli der Stadt Zürich errichtete. Dieser Standort, dessen Benützung dem grossen Entgegenkommen der Stadt Zürich zu verdanken war, ermöglichte das Experimentieren mit Abwasser, das direkt aus der Anlage der Stadt gepumpt werden konnte, so dass wir 


\section{Versuchsanlage Werdhölzli}

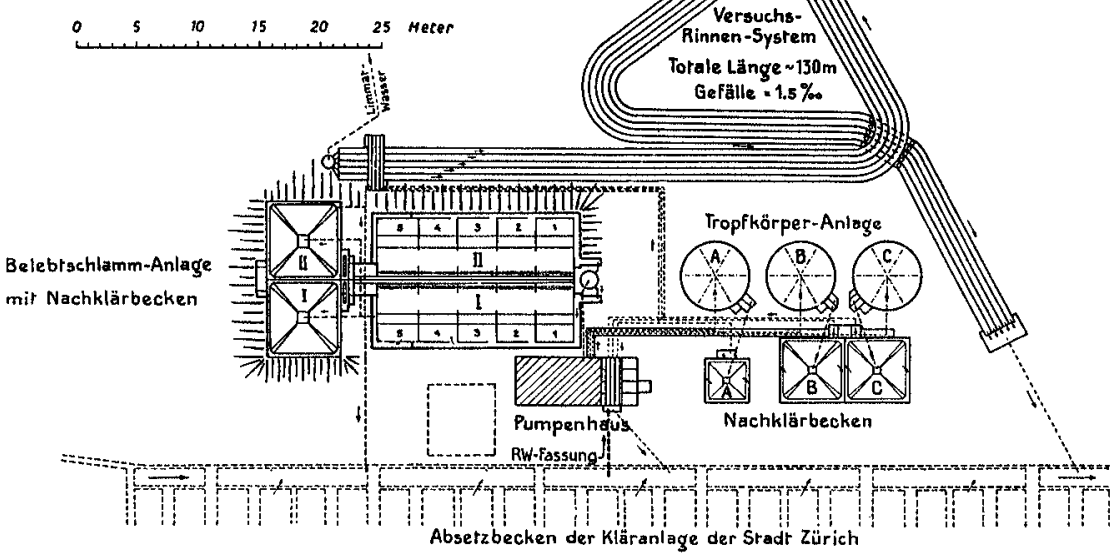

Abb. 2. Halbschematische Darstellung der Anordxung der Versuchsanlagen der EAWAG. im Areal der Kläranlage Werdhölzli der Stadt Zürich.

keine eigenen Vorklärungsbecken zu errichten hatten. Die Gesamtdisposition der benützten Anlageteile der Versuchsstation ist aus Abbildung 2 ersichtlich.

\section{BELEBTSCHLAMMANLAGE}

Die 1937/38 errichtete Belebtschlammanlage, die unter der Leitung von Prof. Dr. E. MEYer-Peter und unter Beratung von Ing. KesSENER (damals Direktor des Niederländischen Reichsinstitutes für Abwasserreinigung) von Ing. A. KROPF errichtet wurde, umfasst folgende, für unsere weiteren Ausführungen wichtigen Teile (für eine eingehende Beschreibung vgl. KROPF, I940):

Zwei baulich identische Einzelanlagen, die vollständig unabhängig voneinander betrieben werden können und somit streng vergleichbare Parallelversuche gestatten.

Jede Anlage besteht aus einer Belïftungskammer der Bauart KESSENER (vgl. Schema und Übersichtsphoto der Abbildungen 3, 4 und 5) von $190 \mathrm{~m}^{3}$ Inhalt, die mittels Querwänden in fünf Abteile $\mathrm{zu}$ je rund $38 \mathrm{~m}^{3}$ gegliedert ist. Die einzelnen Abteile können gesamthaft oder einzeln (nach Verschluss der Verbindungslücken) betrieben werden. Die Experimente mit kurzen Belüftungszeiten wurden nur mit einer oder zwei Kammern 


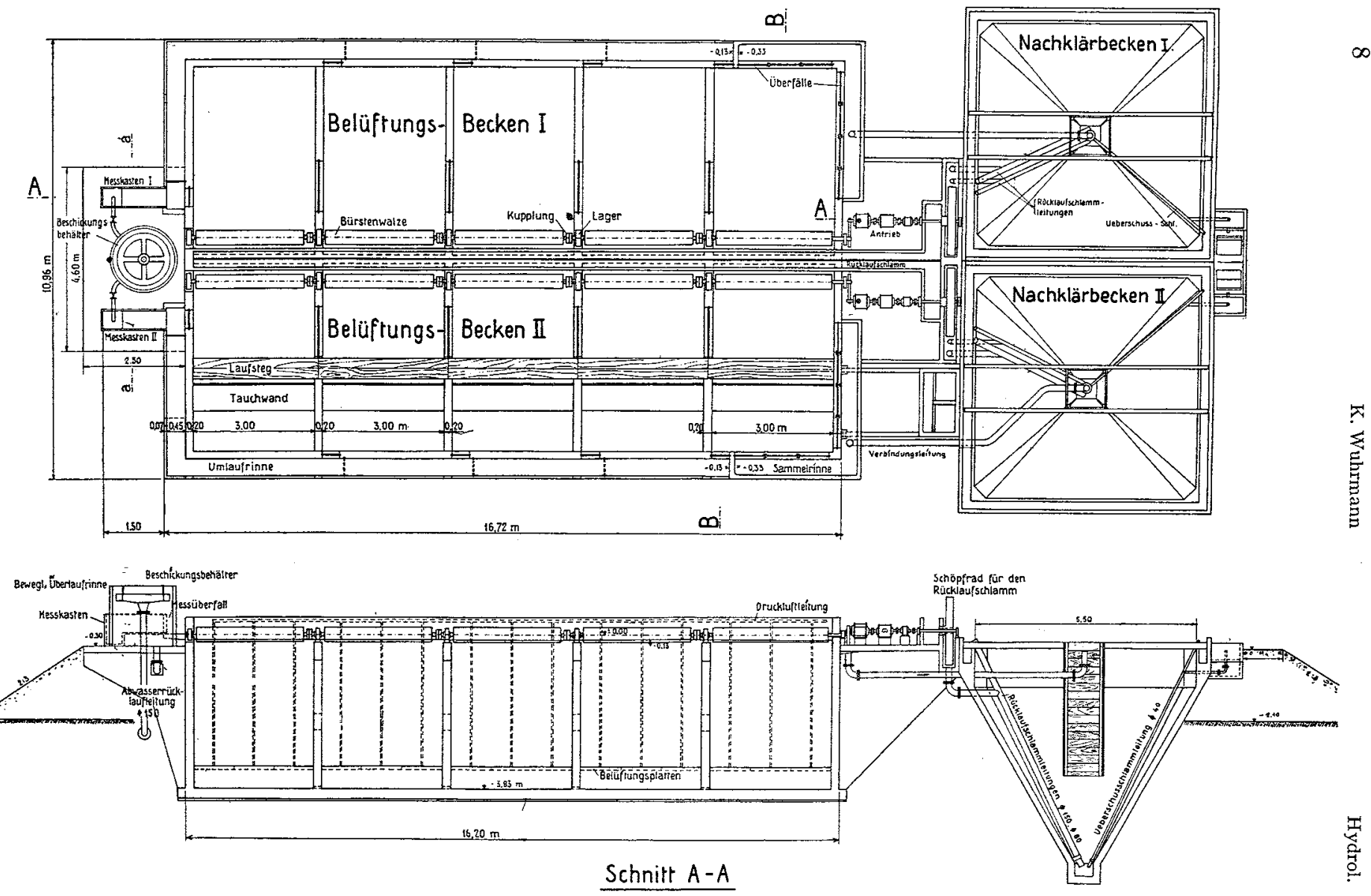

Abb. $3 b$. Grundriss und Schnitt der Belebtschlammaniagen und der zugehörigen Nachklärbecken. 
ausgeführt. Die Belüftung erfolgt als sogenannte Oberflächenbelüftung mittels rotierender Bürsten aus neunteiligen gezähnten Stahllamellen (Abb.5). Der Bürstenantrieb gestattet Tourenzahlen von 70-240 U./min. Durch entsprechende Einstellung der Höhe der Beckenüberlaufkante können Eintauchtiefen der Bürsten von o bis $\sim 9 \mathrm{~cm}$ erreicht werden. Für eventuelle Versuche mit Druckluftbelüftung sind Filterplatten eingebaut.

Jeder Belüftungskammer ist ein Nacbklärbecken (Trichterbecken mit quadratischem Grundriss, Wandneigung $60^{\circ}$ ) nachgeschaltet, in welches das Belebtschlammgemisch mit freiem Gefälle überfliesst. Die Einleitung ins Nachklärbecken erfolgt in einem zentralen Beruhigungskasten, der Abfluss des geklärten Abwassers über Zackenüberfälle aus Eternit am Beckenrande. Der Inhalt der Nachklärbecken beträgt $77 \mathrm{~m}^{3}$, wovon als eigentlicher Absetzraum rund $70 \mathrm{~m}^{3}$ gelten können. Beckenoberfläche $\sim 30 \mathrm{~m}^{2}$, Länge der Überfallkante $22 \mathrm{~m}$.

Der Abwasserzulauf (Rohwasser) erfolgt aus einem gemeinsamen Niveaubehälter für beide Belebtschlammanlagen in je einen Messkasten mit Thompson-Überfall. Von dort wird das Rohwasser in einer seitlichen Rinne den Bècken entlanggeführt und durch entsprechende Öffnungen in die einzelnen Abteilungen der Belüftungskammern eingelassen. Bei Versuchen mit einem einzigen Belüftungsabteil wurde zur Vermeidung von Kurzschlüssen der Beckenzufluss mit einer Rohrleitung an der dem Beckenablauf gegenüberliegenden Wand in etwa halbe Beckentiefe einge-

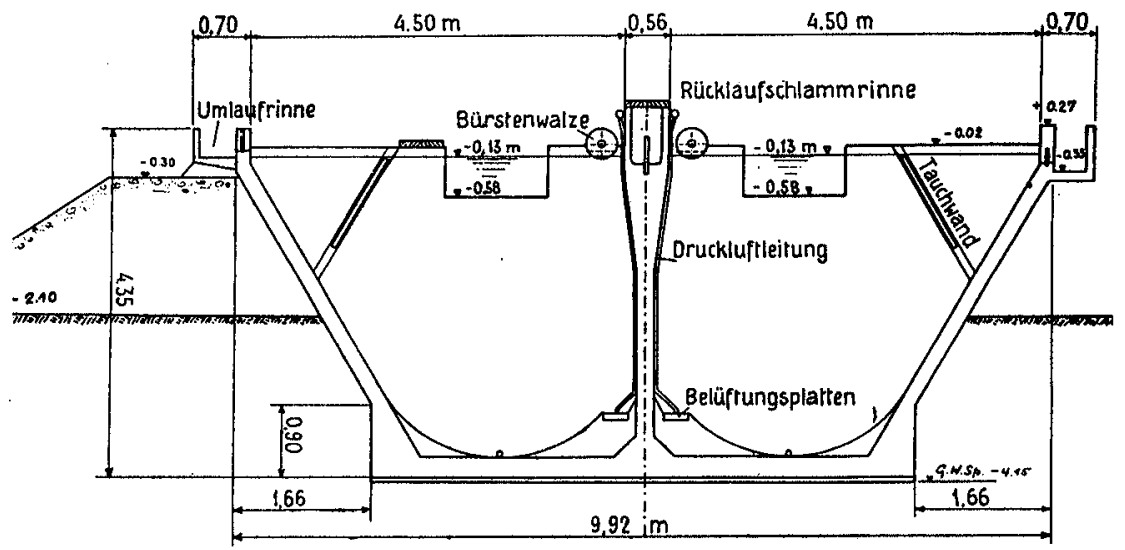

Schnitt B-B

Abb. $3 a$. Schnitt der Belebtschlammanlagen 
führt. In diesem Falle erfolgte der Abfluss aus dem Becken mit einem Tauchrohr aus etwa halber Beckentiefe.

Der Rücklaufscblamm wird aus der Trichterspitze der Nachklärbecken mit Wasserüberdruck in einen zwischen Belüftungs- und Nachklärbecken angeordneten Kasten gefördert, von dort mittels Schöpfrädern über die Spiegelhöhe der Belüftungsbecken gehoben und in Rinnen, die sich zwischen den beiden Belüftungsbecken befinden, in die letztern zurückgeleitet. Der Zufluss des Rücklaufschlammes erfolgt damit auf der dem

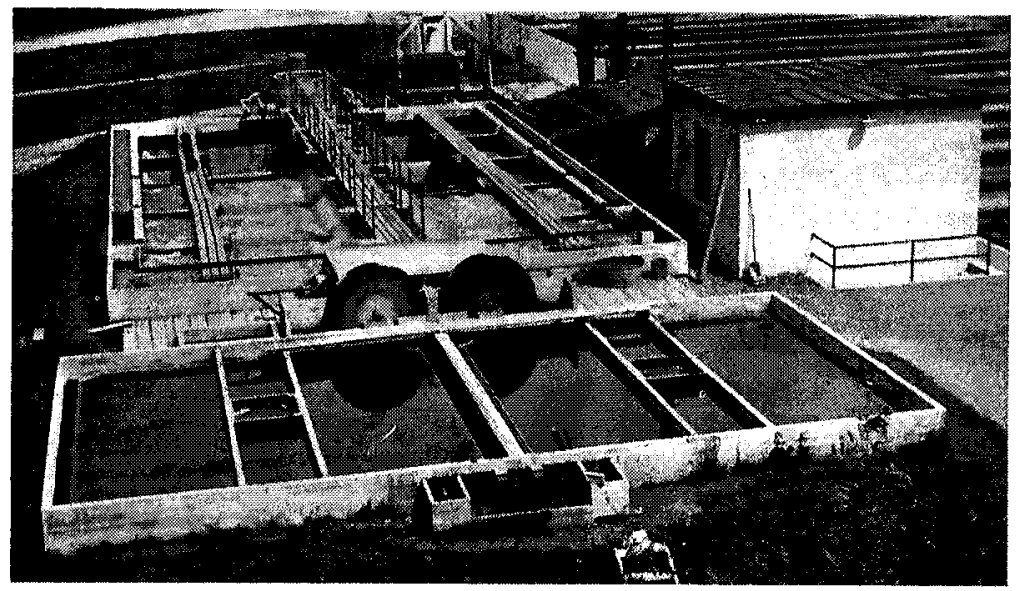

Abb. 4. Überblick über die Belebtschlammanlagen. Vorne die Nachklärbecken, hinten die baiden mehrteiligen Balüftungskammern. Rechts aussen: Südliche Hälfte der Emscherbrunnen der städtischen Anlage.

Rohwassereinlauf gegenüberliegenden Beckenseite. In den letzten Versuchen wurden Rücklaufschlamm und Rohwasser vor dem Eintritt in die Belüftungskammern miteinander vermischt.

Die Messung und Dosierung der Rücklaufschlammenge wird am Austritt der Rücklaufschlammleitung in den Schöpfradkasten mittels kalibriertem Schieber vorgenommen.

Rücklaufschlammförderung und Antrieb der Bürstenwalzen erfolgt für jede Anlage von einem zwischen Belüftungs- und Nachklärbecken angeordneten Elektromotor mit Reduktionsgetriebe aus.

Der Überschußscblamm wurde bei allen beschriebenen Versuchen aus der Rücklaufschlammleitung abgezapft. Seine Messung erfolgte anfänglich mittels Kippmulden und Zählwerk, später mittels Messüberfällen.

Die in jedem einzelnen Versuch verwendeten Einrichtungen sind im übrigen in Tabelle 9 angegeben. 
Die Anlage bewährte sich im Laufe der I4jährigen Versuchszeit im allgemeinen in technischer Hinsicht sehr gut. Die Grösse der Nachklärbecken erwies sich allerdings als begrenzender Faktor für die Experimente, weil ihr beschränkter Inhalt dazu zwang, bei hohen Raumbelastungen nur eine einzige Kammer der Belüftungsbecken zu verwenden, um im Nachklärbecken noch eine genügende Aufenthaltszeit zu erhalten. Die Gefahr

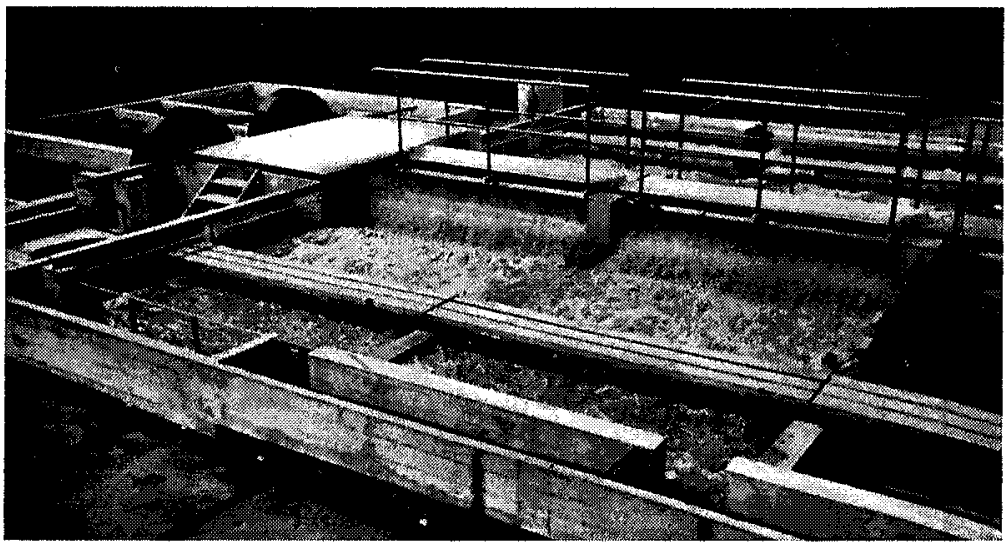

Abb. 5. Dətailansicht von zwei Belüftungsabteilungen der nördlichen Belüftungskammer (in Betrieb). Links hinten die Schöpfräder zur Rücklaufschlammhebung.

von Kurzschlüssen in diesen verkleinerten Belüftungsbecken wurde damit sehr gross.

\section{KÜNSTLICH BELÜFTETER TROPFKÖRPER}

Im Jahre 1944 wurden drei Tropfkörper mit zugehörigen Nachklärbecken errichtet, von denen an dieser Stelle nur der geschlossene, künstlich belüftete beschrieben wird (Abb.6).

Der aus Eisenbeton errichtete, kreiszylinderförmige Tropf körpermantel von $5,5 \mathrm{~m}$ lichtem Durchmesser ist oben mittels einer aufbetonierten Kuppel geschlossen. Die Sohle des Körpers besteht aus parallelen Abflussrinnen, die in einen zentralen, nach aussen führenden Kanal münden. Über jeder Seitenrinne ist ein Luftloch angeordnet, durch welches die von oben her durch das Filterbeet gepresste Luft ausströmen kann. Úber dem Dränagesystem des Tropfkörperbodens ist mittels Formsteinen eine durchlässige Sohle errichtet, auf welche die Tropfkörperfüllung aufgeschichtet ist. Die Belüftung wird mittels eines Ventilators, der im Zenith der Kuppelüberdeckung eingebaut ist, vorgenommen. Die Dosierung der 
Luft erfolgte anfänglich durch Verstellen des «Kaminhutes» des Ventilators und mittels Pitotrohrmessung im Saugrohr. Später wurde diese Einrichtung durch ein regulierbares Gebläse mit vorgeschaltetem Venturikanal zur Luftmengenmessung ersetzt (Serien VIII bis X) ${ }^{\mathrm{I}}$ ).

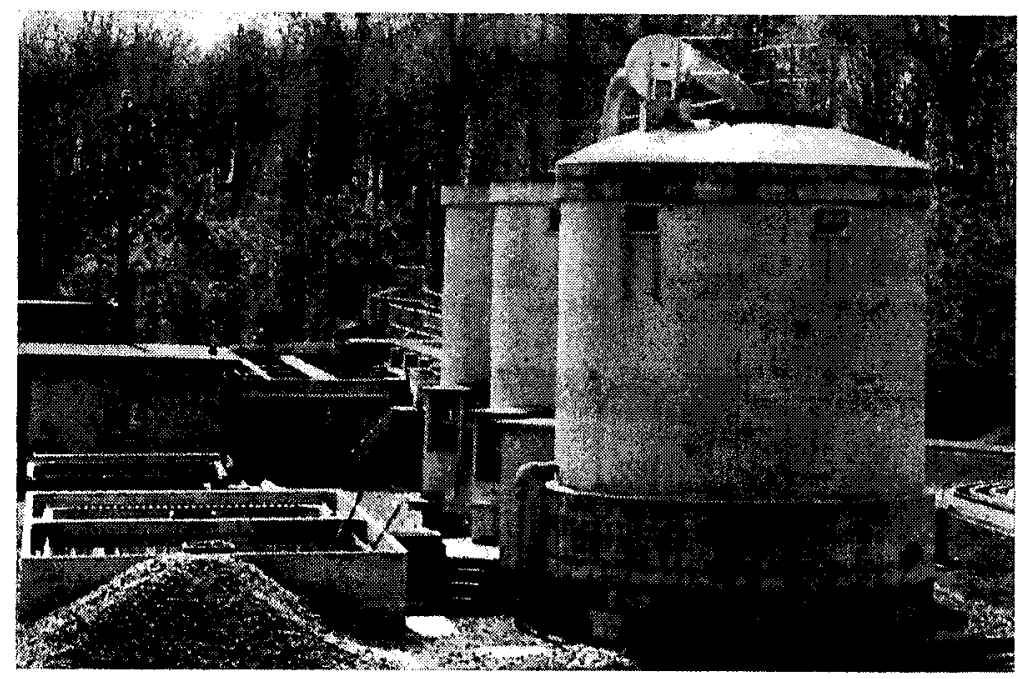

Abb. 6. Ansicht dər drei Versuchstropfkörper. Im Vordergrund der gedeckte, künstlich belüftete Filter mit Ventilator, links Nachklärbecken.

Die Beschickung des Körpers erfolgte durch einen sechsarmigen Drehsprenger; die Wassermenge wurde am Tropfkörperauslauf mit einem Registrierpegel gemessen.

Als Nacblärbecken wurde ein Trichterbecken mit quadratischem Grundriss gewählt. Der Zufluss erfolgt durch einen zentralen Beruhigungskasten, der Abfluss über einen Eternitzackenüberfall am Beckenrande. Totalinhalt des Nachklärbeckens $=47 \mathrm{~m}^{3}$, davon nutzbarer Absetzraum $\sim 38 \mathrm{~m}^{3}$ (restliches Volumen $=$ ruhender Schlamm in der Trichterspitze). Beckenoberfläche $=20 \mathrm{~m}^{2}$, Länge der Überlaufkante $=\sim \mathrm{I} 8 \mathrm{~m}$.

Der Überschußschlamm wird mittels Wasserüberdruck aus dem Nachklärbecken in einen geeichten Meßschacht gefördert und verlässt von dort aus unsere Anlage.

1) Diese Belüftungsvorrichtung wurde mit Unterstützung der Eidgenössischen Stiftung zur Förderung schweizerischer Volkswirtschaft durch wissenschaftliche Forschung finanziert. Wir möchten der Stiftung auch an dieser Stelle für ihren Beitrag nochmals bestens danken. 
Die Füllung des Tropfkörpers wurde mit einem Gemisch von Lavaschlacken und Kupolofenschlacken folgender Körnungen vorgenommen:

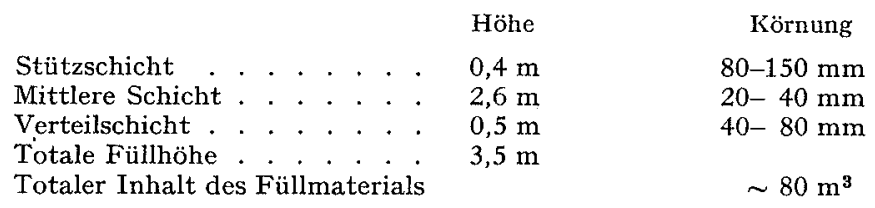

Alle Tropfkörper- und Belebtschlammanlagen werden von einer gemeinsamen Pumpstation aus mit Abwasser gespiesen, das im Ablaufkanal der städtischen Absetzbecken gefasst wird (vgl. Abb.2). Es standen stets so viele Pumpen zur Verfügung, dass jede Versuchseinheit völlig unabhängig von den andern betrieben werden konnte. Das Pumpenhaus dient gleichzeitig auch als Laboratorium für die jeweils an Ort und Stelle vorgenommenen Untersuchungen.

Die Belebtschlamm- und Tropfkörperanlage waren beide sowohl als Experimentier- wie auch als Demonstrationsanlagen entworfen worden. Bei den Versuchsanordnungen musste deshalb mancher Kompromiss geschlossen werden. Anderseits können wir die Dimensionen beider Installationen als genügend gross betrachten, um die damit gemachten Erfahrungen ohne Einschränkungen auf ähnliche oder grössere Bauwerke der Praxis übertragen zu können (soweit es sich um analoge Abwasserverhältnisse handelt!). Zur Illustration geben wir die Einwohnergleichwerte für die Abwassermengen an, die wir bei den häufigsten Versuchen behandelten (Tabelle I).

Tabelle 1

Bebandelte Abpassermengen in Einmobnergleicbwerten bei den bäufigsten Versucbsanordnungen

\begin{tabular}{|c|c|c|c|c|c|c|c|c|}
\hline & \multicolumn{4}{|c|}{ Belebtschlammanlage } & \multicolumn{4}{|c|}{$\begin{array}{l}\text { Künstlich belüfteter } \\
\text { Tropfkörper }\end{array}$} \\
\hline & \multicolumn{4}{|c|}{$\begin{array}{l}\text { Angenommene spezifische } \\
\text { Abwassermenge }(1 / \mathrm{E} \cdot \mathrm{Tag})\end{array}$} & \multicolumn{4}{|c|}{$\begin{array}{l}\text { Angenommene spezifische } \\
\text { Abwassermenge }(1 / \mathrm{E} \cdot \mathrm{Tag})\end{array}$} \\
\hline & 200 & 450 & 200 & 450 & 200 & 450 & 200 & 450 \\
\hline $\begin{array}{l}\text { Verwendeter Belüftungs- } \\
\text { raum }\left(\mathrm{m}^{3}\right) \text {. } \cdot . \cdot . \\
\text { Hydraulische Raum- } \\
\text { belastung } \\
R_{h}\left(\mathrm{~m}^{3} / \mathrm{m}^{3} \cdot \text { Tag) . . }\right.\end{array}$ & 10 & 10 & 38,5 & 38,5 & 80 & 80 & 80 & 80 \\
\hline $\begin{array}{l}\text { Einwohnergleichwert der } \\
\text { behandelten Abwasser- } \\
\text { menge. . . . . . . . }\end{array}$ & 3900 & 1750 & 4000 & 1900 & 7950 & 875 & 3120 & 1400 \\
\hline
\end{tabular}




\section{Betrieb der Versuchsanlagen}

\section{ROHWASSER}

In allen nachiolgend geschilderten Versuchen wird als "Rohwasser» (RW) der Abfluss der südlichen Hälfte der städtischen Kläranlage benützt. Diese Absetzanlage wurde von MüLLER (I929) eingehend beschrieben. Sie besteht im wesentlichen aus Sandfang, automatisch gereinigtem Rechen, erstem flachem Absetzbecken mit automatischer Schlammräumung und anschliessendem, längsdurchflossenem Absetzbecken mit abgetrenntem Schlammraum (Travisbecken). Der letztere wird lediglich als Sammel- und Stapelraum benützt; der Schlamm wird regelmässig in besondere Faulkammern zur Weiterverarbeitung gepumpt. Der Abfluss aller Absetzbecken einer Anlagehälfte fällt in einen gemeinsamen Kanal über, aus welchem wir für unsere Anlage das vorgereinigte Rohwasser entnahmen. Mit Hilfe einer scharf ausgeprägten und jeden Morgen auftretenden Spitzenkonzentration der Chloride im städtischen Abwasser haben wir mehrmals die wirkliche Durchflusszeit durch die städtische Vorklärung (südlicher Teil) bestimmt und um 9 Uhr Werte von 40-45 min gefunden. Bei Trockenwetter liegt der Abwasserzufluss um diese Zeit etwas über dem Tagesmittel. Für einzelne Versuche mit dem künstlich belüfteten Tropfkërper wurde jeweils bei einem städtischen Becken die Aufenthaltszeit durch Drosselung des Zuflusses verlängert, um eine bessere Vorklärung zu erhalten. Der Einfluss dieser Massnahme erwies sich jedoch als gering, wie dies Mittelwerte aus einer längeren Bestimmungsreihe (Serie $\mathrm{X}, \mathrm{TK}$ ) in Tabelle 2 zeigen.

Das Abwasser der Stadt Zürich, wie es für die Versuche verwendet werden musste, ist relativ wenig konzentriert und lässt sich gut mit den Verhältnissen vergleichen, wie sie in den grossen Städten der USA. vorliegen. Es erweist sich damit als bedeutend "dünner» als beispielsweise die durchschnittlichen städtischen Abwässer in Deutschland oder England.

Da die ersten in diesem Bericht berücksichtigten Versuche in die Zeit strengster Lebensmittelrationierung in den letzten Kriegsjahren fielen, besass das zur Verfügung stehende Abwasser eine besonders geringe Konzentration (Serien V, VI, VII). Speziell die Kennzahlen $\mathrm{Bsb}_{5}$ und $\mathrm{KMnO}_{4}{ }^{-\prime}$ Verbrauch widerspiegeln deutlich den Zusammenhang zwischen Ernährungsgewohnheiten und Abwasserkonzentration in den Kriegsjahren. Die eingehenden Untersuchungen in der letzten Zeit konnten dagegen mit Abwasser durchgeführt werden, dessen Konzentration als normal für 
Tabelle 2

Einfluss der Aufentbaltszeit in den städtiscben Vorklärbecken auf die Qualität des Beckenabflusses

(Tagesmittel aus Serie X, TK)

\begin{tabular}{|c|c|c|}
\hline & \multicolumn{2}{|c|}{$\begin{array}{c}\text { Aufenthaltszeit (Rechen bis Beckenabfluss, } \\
\text { gemessen an Chloridspitze) bei } \\
\text { Trockenwetter um } 9 \text { Uhr }\end{array}$} \\
\hline & $\sim 40 \min$ & $\sim 85 \min$ \\
\hline $\begin{array}{l}\text { Trübung, nephelometrisch (Millimeter } \\
\text { Galvanometerausschlag) }\end{array}$ & 60.5 & 56,5 \\
\hline Trockenrückstand . . . . (mg/l) & 549 & 535 \\
\hline Glühverlust & 222 & 220 \\
\hline Schwebestoffe & 151 & 139 \\
\hline Oxydierbarkeit & 340 & 322 \\
\hline $\mathrm{Bsb}_{5} . \ldots$. & 139 & 130 \\
\hline Gesamtstickstoff . & 23,6 & 25,0 \\
\hline Gesamtphosphor . . & 2,9 & 2,8 \\
\hline Organischer Kohlenstoff...$(\mathrm{mg} \mathrm{C} / 1)$ & 82,1 & 81,9 \\
\hline
\end{tabular}

Gemeinden mit hoher spezifischer Abwassermenge bezeichnet werden kann.

Die tägliche Schwankung von Abwassermenge und -konzentration in der Kläranlage Werdhölzli der Stadt Zürich spielte für die Durchführung der Experimente und speziell die Technik der Probenahme (vgl. nächsten Abschnitt) eine grosse Rolle. Zahlreiche Auswertungen von Abflusskurven bei Trockenwetter (Limnigraph am städtischen Hauptsammelkanal) ergaben:

a) Das tägliche Maximum des Zuflusses zur Kläranlage tritt im allgemeinen in zwei Gipfeln zwischen I2 und I4 Uhr auf; das Minimum zwischen 2 und 6 Uhr.

b) Der mittlere tägliche Abfluss beträgt $\sim \mathrm{I}, 5-\mathrm{I}, 6 \mathrm{~m}^{3} / \mathrm{s}$, die täglichen Minima und Maxima bei Trockenwetter weichen $\sim 30-40 \%$ vom Tagesmittelwert $a b$.

c) Das Verhältnis zwischen Minimal- und Maximalabfluss ist bei Trockenwetter rund $\mathrm{r}: 2$.

Wir haben mehrfach auch die Konzentrationsschwankung des Abwassers durch stündliche oder zweistündliche Probenahme am Abfluss der Vorklärbecken (= Zufluss zur Versuchsanlage) festgehalten. Als Beispiel geben wir die Diagramme der Abbildung 8. Darin sind die pro Zeiteinheit aus der städtischen Anlage abfliessenden Schmutzstoffmengen an zwei 
Stichtagen mit Trockenwetterabfluss in der städtischen Kanalisation aufgetragen. Es ergibt sich, dass das Maximum des Abflusses von Stickstoffverbindungen rund $2-3 \mathrm{~h}$ früher auftritt als das Maximum des $\mathrm{Bsb}_{5}$. Die Kurve für den Phosphor folgt mehr oder weniger derjenigen des Stickstoffs. Es ist auffallend, dass - abgesehen von Details - die zeitliche Verteilung der Schmutzstoffkonzentration und der Einzelkomponenten sehr regelmässig erfolgt und in weit auseinanderliegenden Kontrollen immer mehr oder weniger gleichartig befunden wurde.

Das in den Versuchen verarbeitete Rohwasser lässt sich im weitern noch durch die in Tabelle 3 als Beispiele zusammengestellten Verbältniszablen zwiscben Bsb $b_{5}$ and Gesamtstickstoff bzw. organischem C und Gesamtstickstoff sowie des gegenseitigen Verhältnisses der Stickstoffkomponenten charakterisieren. Vergleichsweise haben wir die entsprechenden Ziffern einiger ausländischer Anlagen eingetragen. Im Hinblick auf die späteren Ausführungen ist zu bemerken, dass die Abwassereigenschaften in einigen amerikanischen Städten weitgehende Ähnlichkeiten mit den unsern aufweisen (vgl. Beispiele in Tabelle 3). Im übrigen wird im Abschnitt III die Bedeutung der angeführten Verhältniszahlen zu diskutieren sein.

Besonders wichtig ist die Verteilung der Schmutzstoffe auf gelöste und ungelöste Anteile im Robwasser nach Durchfluss der städtischen Kläranlage. In Tabelle 4 geben wir Analysen (Tagesmittelproben) des Rohwassers an, wie es in unsere Anlage gepumpt wurde, sowie vom selben Rohwasser nach Filtration durch Membranfilter (Sterilfiltration). Es ist sofort er-

Tabelle 3

Verbältniszablen von Robwasserkomponenten

\begin{tabular}{|c|c|c|c|c|c|}
\hline & \multicolumn{2}{|c|}{$\begin{array}{l}\text { Werdhölzli, Abfluss } \\
\text { städtische Anlage }\end{array}$} & \multirow{2}{*}{$\begin{array}{l}\text { Chicago } \\
\text { SW }\end{array}$} & \multirow{2}{*}{$\begin{array}{l}\text { Chicago } \\
\mathrm{N}\end{array}$} & \multirow{2}{*}{$\begin{array}{l}\text { Jackson, } \\
\text { Michigan }\end{array}$} \\
\hline & 1949 & 1950 & & & \\
\hline \multirow{8}{*}{$\begin{array}{l}\mathrm{Bsb}_{5} \text { : Gesamtstickstoff } \\
\text { Organischer Kohlen- } \\
\text { stoff: Gesamtstickstoff } \\
\text { Stickstoffkomponenten: } \\
\text { OrganischerStickstoff in } \\
\text { Prozent des Gesamt- } \\
\text { stickstofs . . } \\
\mathrm{NH}_{4}-\mathrm{N} \text { in Prozent des } \\
\text { Gesamtstickstoffs . } \\
\left(\mathrm{NO}_{2}+\mathrm{NO}_{3}\right)-\mathrm{N} \text { in Pro- } \\
\text { zent des Gesamtstick- } \\
\text { stoffs....... }\end{array}$} & $4,9: 1 \div 4,5: 1$ & $5,8: 1$ & $6,2: 1$ & $6: 1$ & $6,6: 1$ \\
\hline & $2: 1 \div 17: 1$ & & & & \\
\hline & $2.1 \div 1,1.1$ & , & & & \\
\hline & & & & & \\
\hline & $28,3 \div 29,1$ & 42,2 & 59 & 49 & 40 \\
\hline & & & & & \\
\hline & $03 \div 01$ & 03 & 41 & 51 & 60 \\
\hline & $4,7 \div 5,9$ & 2,8 & 0 & 0 & 0 \\
\hline
\end{tabular}


Tabelle 4

Anteil der Schwebestoffe an den Komponenten des Robwassers im Abfuss der städtiscben Anlage (Herbst 1950)

\begin{tabular}{|c|c|c|c|}
\hline & $\begin{array}{l}\text { Abfluss } \\
\text { städtische } \\
\text { Anlage }\end{array}$ & $\begin{array}{c}\text { Nach } \\
\text { Steril- } \\
\text { filtration }\end{array}$ & $\begin{array}{c}\text { In Schwebe- } \\
\text { stoffen } \\
\text { festgelegt }\end{array}$ \\
\hline 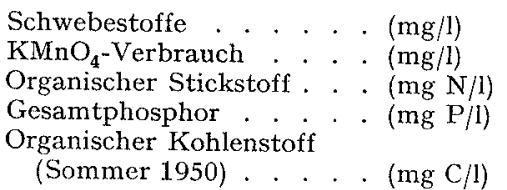 & $\begin{array}{l}120 \\
314 \\
8,82 \\
1,99 \\
82,1\end{array}$ & $\begin{array}{c}0 \\
133 \\
4,72 \\
0,91 \\
20,9\end{array}$ & $\begin{array}{l}-\overline{-6} \% \\
46,5 \% \\
54,3 \% \\
74,6 \%\end{array}$ \\
\hline
\end{tabular}

sichtlich, dass ein beträchtlicher Teil des Schmutzstoffgehaltes in suspendierter Form im Rohwasser enthalten ist, speziell hohe Anteile entfallen auf den organischen Kohlenstoff in den Schwebestoffen. Im allgemeinen bewegte sich der Gehalt an Schwebestoffen in unserem Rohwasser um roo-r2o mg/l (vgl. Analysen in Tabelle II).

Unsere Versuchseinrichtungen erlaubten nicht, den täglichen Gang der Abflussmenge des Abwassers in der städtischen Kläranlage auf die Experimente exakt zu übertragen, und diese Variable war auch im allgemeinen bei der Versuchsdurchführung nicht erwünscht. In vielen Experimenten wurde als Ersatz für den natürlichen Wechsel des Rohwasserzuflusses ein vereinfachtes Schema der Tagesschwankungen bei der Beschickung der Versuchsanlagen durchgeführt, oder es wurde der Einfachheit halber mit konstanter Wassermenge gearbeitet. Man kann sich fragen, wie weit durch diese vereinfachte Beschickung der Versuchsanlagen ihre Belastung mit Schmutzstoffen im Vergleiche mit normalen Gemeindeanlagen beeinflusst wird. Wir prüfen dies wie folgt:

Wir nehmen als Beispiel für einen "Normaltag» bezüglich Abwasserund Schmutzstoffmenge im Abfluss der städtischen Kläranlage die Beobachtungen vom I9. und 20.Januar 1949 (Abb.7) und rechnen diese Ziffern für einen Tagesmittelabfluss von $\mathrm{I} \mathrm{m}^{3} / \mathrm{s}$ um. In Abbildung 8 haben wir mit dem gleichen Maßstab (Tagesmittel $=\mathrm{I} \mathrm{m}^{3} / \mathrm{s}$ ) die Zuflussmenge $z u$ unsern Anlagen in den einzelnen Versuchsserien aufgezeichnet. Desgleichen wurden die entsprechend der wechselnden Abwassermenge deformierten Kurven für den Schmutzstoffzufluss eingetragen. Es ergibt sich aus der Darstellung ohne weiteres, dass zum Beispiel eine ïber $24 \mathrm{~h}$ gleichmässige Beschickung die Maxima und Minima in den Schmutzstoff- 
mengen etwas ausgleicht, während besonders die zweistufige Beschickung viel höhere Spitzenbelastungen mit sich bringt, als dies bei einer Gemeindeanlage der Fall sein würde (Serie BS IX, X und TK IX und X). Ganz extreme Bedingungen ergeben sich bei noch grösserem Verhältnis zwischen Tages- und Nachtzufluss (BS $V_{6}$ und $V_{5}$ bzw. TK $V_{5}$ und $V_{6}$, $\mathrm{VIII}_{4}$ und $\mathrm{VII}_{6}$ ). Integriert man aber die Kurven für die einzelnen Schmutz-

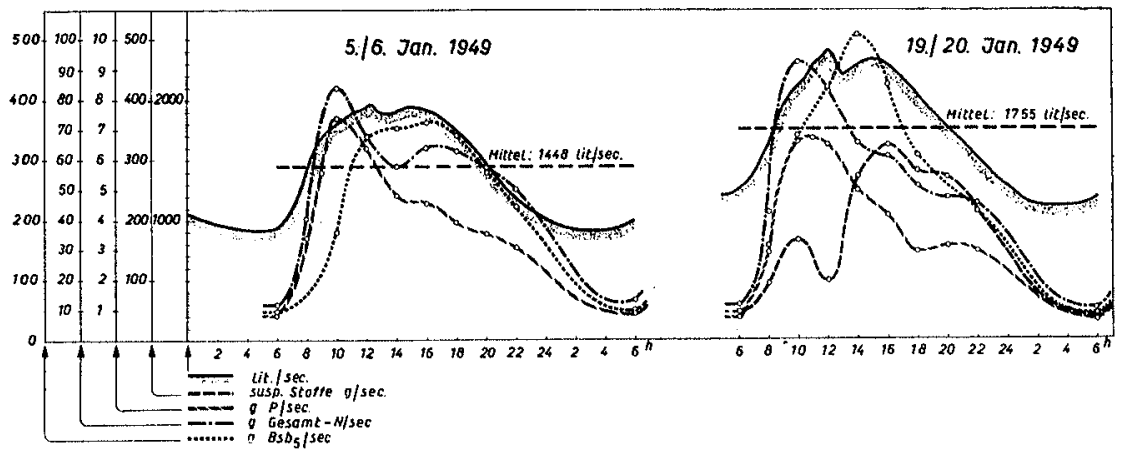

$\mathrm{Abb}$. 7. Abflusskurven für Schmutzwasser, suspendierte Stoffe, Bsb $\mathrm{B}_{5}$, Gesamtstickstoff und -phosphor aus den südlichen Klärbecken der städtischen Kläranlage Werdhölzli, Zürich. Die Kurven vom 19./20. Januar 1949 betrachten wir für spätere Berechnungen als typisch für einen "Normaltag".

Ordinaten: Wasser- bzw. Stoffmengen in $\mathrm{m}^{3} / \mathrm{s}$ bzw. g/s. Abszisse: Tagesstunden.
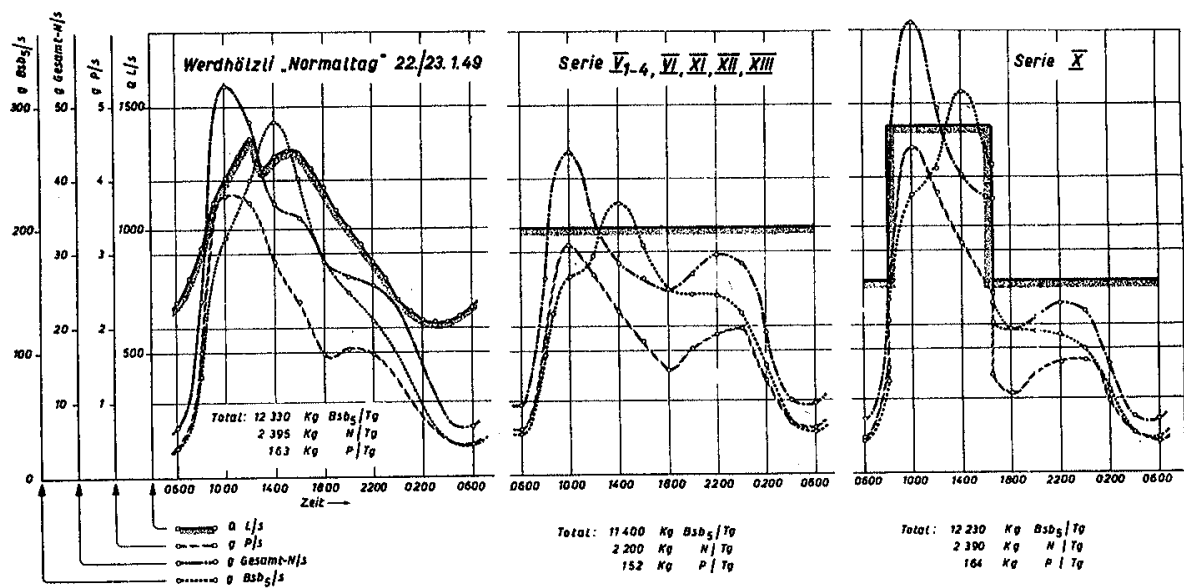

Abb. 8. Abwasser- und Schmutzstoffzufluss zu den Versuchsanlagen bei verschiedenen Experimenten im Vergleich mit der natürlichen Tageskurve im Abflusskanal der städtischen Kläranlage bei Trockenwetter.

Ordinaten: Relative Abwassermenge (1/s) und Schmutzstoffmenge (g/s), wenn der Tagesmittelabfluss $2 u 1 \mathrm{~m}^{3} / \mathrm{s}$ angenommen wird. Abszisse: Tagesstunden. 
stoff kennzahlen über $24 \mathrm{~h}$, so sind die Tagessummen bei den einzelnen schematischen Beschickungsarten nur unwesentlich verschieden vom effektiven Schmutzstoffabfluss der städtischen Kanalisation innerhalb des gleichen Zeitraums. Wir sind desbalb der Auffassung, dass trotz der Vereinfacbung in der Bescbickungsweise unserer Versucbsanlagen die gemonnenen Resultate obne Einscbränkung auch gültig sind für Anlagen, denen das Abwasser gemäss einer natürlichen Abflusskurpe der Gemeindekanalisation zufliesst.

\section{DURChFÜHRUNG DER Versuche}

Grundsätzlich wurde bei allen Experimenten eine mindestens mehrwöchige Beobachtungsperiode eingehalten und die Anlagen in dieser Zeit gemäss einem vorbestimmten Schema bezüglich aller Betriebsdaten obne jede Änderung betrieben. Innerhalb dieser Versuchsperiode erfolgte die in jedem einzelnen Fall als notwendig erachtete Anzahl von Probenahmen. Ein tägliches Betriebsprotokoll, das alle wichtigen technischen Angaben über den Gang der Versuchsanlagen enthielt, diente zur Überwachung der Experimente.

Im allgemeinen wurden die Belebtschlammanlagen für jede Versuchsphase neu «eingefahren». Dabei hat sich folgendes Schema bewährt:

a) Füllen der Belüftungsbecken mit Rohwasser und Belüftung während $48 \mathrm{~h}$.

b) Nach 48 h Beginn des Rohwasserdurchflusses, wobei innerhalb zirka Io Tagen in gleichmässiger Steigerung die Vollbelastung erreicht wird. Die Rücklaufschlammenge wird von Anfang an auf der für den Vollbetrieb geplanten Höhe gehalten.

c) Der Ablass von Überschußschlamm beginnt erst, wenn die Schlammkonzentration im Belüftungsbecken die gewünschte Höhe erreicht hat.

d) Nach Ablauf der zirka zwölftägigen Einlaufperiode wurde die Anlage vor Beginn der Untersuchungen noch während rund to Tagen sich selbst überlassen. Es zeigte sich, dass nach etwa is Tagen (vom Beginn des Einfahrens an gerechnet) keine Änderungen mehr in den Anlagenleistungen eintraten.

Beim künstlicb belüfteten Tropfkörper erfolgte das Einfahren innerhalb desselben Zeitraumes durch regelmässige tägliche Steigerung des Abwasserdurchflusses. Alle nachfolgenden Analysen beziehen sich aber auf Beobachtungsperioden, bei denen der Tropfkörper schon vorher mehrere Wochen lang in Betrieb war und nur noch die gewünschte Versuchsbelastung genau eingestellt werden musste. 
Vor und während der Beobachtungsperioden wurden auf den Anlagen die normalerweise notwendigen Reinigungsarbeiten vorgenommen. Im übrigen beschränkte sich die Wartung auf die Kontrolle der in den Versuchsprogrammen vorgeschriebenen Betriebsdaten und die Durchführung der gewünschten Messungen. Der Umfang der letzteren war naturgemäss wesentlich grösser, als dies für den Betrieb einer Gemeindekläranlage notwendig ist, und wir möchten deshalb mit Nachdruck darauf hinweisen, dass die von uns regelmässig vorgenommenen Messungen zur rein betriebsmässigen Überwachung einer Gemeindeanlage entbehrlich sind (vgl. auch Abschnitt III, A I).

Obschon grösstes Gewicht darauf gelegt wurde, zwischen den einzelnen Versuchsabschnitten nur diejenigen Variablen der Betriebsführung zu wechseln, deren Einfluss untersucht werden sollte, war es doch nicht zu vermeiden, dass gleichzeitig auch eine Reihe anderer Faktoren von Abschnitt zu Abschnitt änderten (mittlere Rohwasserkonzentration, mittlere Schlammkonzentration, Temperatur des Abwassers usw.). Wie sich jedoch im III. Teil zeigen wird, sind die damit in die Mittelwerte ganzer Versuchsserien hineingetragenen Streuungen geringer als die durch die täglichen Schwankungen der Rohwasserkonzentration ohnehin bedingte Variabilität, so dass der Einfluss der jeweils geprüften Faktoren doch zum Ausdruck gebracht werden konnte.

\section{Probenahme}

Die Erhebung von Proben des Rohwassers und der Anlagenabflüsse, welche bezüglich der Fragestellung eines Versuches oder eines Analysenzieles repräsentativ sind, ist ein auch heute noch unbefriedigend gelöstes Problem. An Hand vieler Untersuchungen, die speziell im Hinblick auf die Probenahmetechnik auf unseren Versuchsanlagen ausgeführt wurden, hat ZEHENDER (1948) die grundlegenden Forderungen formuliert. Wir greifen hier die bei den Experimenten berücksichtigten, wichtigsten Punkte nochmals heraus und verweisen im übrigen auf diese Arbeit.

Je nach Fragestellung wurden folgende Probenahmemethoden verwendet:

a) Sammelproben wäbrend 24 b: Für die Messung des Gesamtumsatzes einer Abwasserkomponente während $24 b$ (Reinigungs- und Eliminationseffekte, Stickstoffbilanz usw.).

Zur Aufsammlung dieser Proben wurde das Abwasser von der Probenahmestelle aus in ein genau horizontal aufgestelltes Überfallgefäss geleitet. Eine kleine Menge des UUberlaufes gelangte in einen grossen Sammelbehälter, der Rest in die Kanalisation. Der Inhalt des Sammelgefässes 
reichte aus, um einen kontinuierlichen Zufluss der Probewassermenge während 24-48 h aufzufangen. Für die Analysen wurde aus dem Gefäss nach gründlichem Umrühren die benötigte Wassermenge erhoben. Diese Vorrichtung bewährte sich sehr gut (Verstopfungen können nicht eintreten, weil eine relativ grosse Wassermenge in das Überfallgefäss eingeleitet werden kann). Die Methode besitzt aber den Nachteil, dass das aufgefangene Abwasser je nach Dauer der Sammelperiode längere Zeit steht, bevor es zur Analyse gelangt. Wir haben die dabei unvermeidlich eintretenden Veränderungen in Kauf genommen, da wir anderseits dank dieser einfachen Probenahmetechnik relativ häufig Tagesbilanzen untersuchen konnten. Eine Aufteilung der 24stündigen Probenahme zum Beispiel in 4- oder 6stündige Einzelperioden, die ebenfalls denkbar wäre, hätte eine untragbare Vervielfachung der Untersuchungsarbeit verursacht, ohne einen entsprechenden Gewinn an Präzision der Messungen zu erbringen, wie dies an zahlreichen Kontrollen festgestellt wurde (vgl. ZEHENDER, 1948). Weitere in Anlehnung an unsere Arbeiten durchgeführte Untersuchungen über die Erhebung von Proben in Kläranlagen erfolgten durch JAEGERS und Mitarbeiter (I95I), der unsere Auffassungen bestätigt. Bei Versuchen mit wechselndem Abwasserzufluss zu den Anlagen (Serie X usw.) wurden die Sammelproben für die Perioden mit verschiedener Anlagenbelastung getrennt voneinander erhoben und für die Analysen im Verhältnis der Beschickungsmenge miteinander gemischt.

b) Einzelproben: Zur vergleichenden Untersuchung der Qualität pon Abflüssen. aus parallel betriebenen Anlagen (meistens zapeimal täglicb erbohen).

Da die täglichen Konzentrationsschwankungen im Abfluss einer biologischen Reinigungsanlage sehr viel geringer sind als im ungereinigten Abwasser (vgl. Abb. 9), ist diese Methode innerhalb eines begrenzten Kreises von Problemstellungen zulässig. Die Abbildung 9 zeigt aber, dass es ausgeschlossen ist, zum Beispiel mittels Einzelproben den Reinigungseffekt einer Anlage bestimmen zu wollen, es sei denn, man erhebe sie während $24 \mathrm{~h}$ in kurzen Zeitabständen.

Die Versuche von Serie XII und XIII wurden lediglich mit zwei täglichen Einzelproben untersucht, da es sich dabei nur um die Kontrolle des $V$ erbaltens einer Anlage bei verschiedenen Bedingungen, nicht aber um die Feststellung der Reinigungsleistung handelte. Einzelproben sind ferner unumgängilich, wenn beispielsweise das Verhalten bestimmter, leicht zersetzbarer Substanzen des Rohwassers bei der Abwasserreinigung verfolgt werden soll (Serie XII: Phenol). 


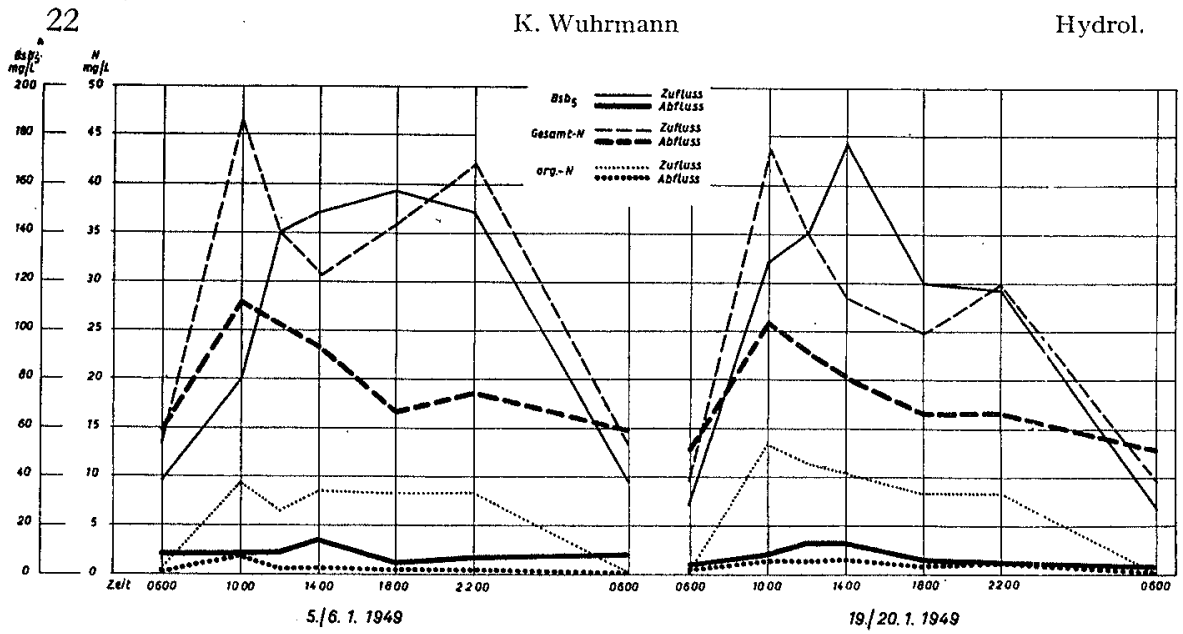

Abb. 9. Baispiel des täglichen Konzentrationsganges des $\mathrm{Bsb}_{5}$, des Gesamtstickstoffs und des organischen Stickstoffs im Zu- und Abfluss der Belebtschlammanlage. Raumbelastung $R_{h}=10 \mathrm{~m}^{3} / \mathrm{mm}^{3} \cdot \mathrm{Tag}$.

Ordinaten: Konzentration des $\mathrm{Bsb}_{5}(\mathrm{mg} \mathrm{O} / \mathrm{l})$ bzw. Stickstoffs (mg N/l). Abszisse: Tagesstunden.

Die Probenahmen (Einzel- und Sammelproben) erfolgten jeweils während einer ganzen Versuchsreihe immer an denselben Punkten der Anlagen, und die Verarbeitung der Proben wurde sofort vorgenommen.

c) Voraussetzung für die Vergleichbarkeit von Proben aus Anlagenzu- und abfluss ist ibre zeitliche Korrespondenz.

Die Erfahrung zeigte, dass die rechnerisch ermittelten Durchflusszeiten durch eine Anlage mit der Wirklichkeit oft sehr wenig übereinstimmen. Die Messung der «effektiven» Durchflusszeit durch eine Kläranlage (bei praktischem Betrieb mit Schmutzwasser) ist recht schwierig. Insbesondere fallen jene Messmethoden weg, die darauf beruhen, eine bestimmte Menge einer in den Anlagenzulauf gegebenen Markierungssubstanz am Anlagenablauf wieder quantitativ zu erfassen. Alle Markierungsstoffe werden mehr oder weniger stark von Schlamm absorbiert und sinken in Klärbecken zu Boden. Die Definition der Durchflusszeit mittels der Lage des Schwerpunktes der Durchflussfläche einer Markierungssubstanz, wie sie von MüLlER-NeuHaus (1952) beschrieben wird, konnte deshalb nicht angewendet werden. Auf Grund unserer praktischen Beobachtungen definieren wir die effektive Durchflusszeit durch unsare Becken mit der zeitlichen Verscbiebung eines leicht erkennbaren Referenzpunktes auf der Durchflusskurve einer (natürlichermeise) im Abwasser vorbandenen Substanz. Als sehr geeignet hat sich die Chloridkonzentration erwiesen. Als Referenzpunkte verwendeten wir 
entweder den Fuss oder das Maximum der jeden Morgen im Rohwasser auftretenden Chloridspitze.

Die bei verschiedenen Betriebsweisen der Belebtschlammanlage vorhandenen Abweichungen aus rechnerischen und wirklichen Durchflusszeiten sind in Tabelle 5 zusammengestellt, wobei die «theoretische Auf-

Tabelle 5

Theoretische und beobacbtete Durcbflusszeiten der Versucbsanlagen bei perschiedenen Raumbelastungen

\begin{tabular}{|c|c|c|c|c|c|c|c|c|c|c|c|}
\hline \multirow[b]{2}{*}{ Serie } & \multirow{2}{*}{$\begin{array}{c}V \underset{\mathbf{1}^{\prime}}{\mathrm{BB}} \\
\mathrm{m}^{3}\end{array}$} & \multirow{2}{*}{$\begin{array}{c}V \\
\mathrm{NKB}^{\prime} \\
\mathbf{2}^{2} \\
\mathrm{~m}^{3}\end{array}$} & \multirow{2}{*}{$\begin{array}{c}Q \mathrm{RW} \\
1 / \mathrm{s}\end{array}$} & \multirow{2}{*}{$\mid \begin{array}{c}Q \mathrm{RS} \\
1 / \mathrm{s}\end{array}$} & \multicolumn{3}{|c|}{$\begin{array}{l}\text { Durchflusszeit } \\
\text { gemessen }\end{array}$} & \multicolumn{3}{|c|}{$\begin{array}{l}\text { Durchflusszeit } \\
\text { theoretisch }\end{array}$} & \multirow{2}{*}{$\begin{array}{l}\text { Messung } \\
\text { mittels }\end{array}$} \\
\hline & & & & & $\begin{array}{l}t_{\mathrm{BB}} \\
\min \end{array}$ & $\begin{array}{l}t_{\mathrm{NKB}} \\
\min \end{array}$ & $\begin{array}{r}t_{t o t} \\
\min \end{array}$ & $\begin{array}{l}t_{\mathrm{BB}} \\
\text { min }\end{array}$ & $\begin{array}{l}t_{\mathrm{NKB}} \\
\min \end{array}$ & $\begin{array}{l}{ }^{t} t o t \\
\min \end{array}$ & \\
\hline II & 77 & 70 & 19 & 8,8 & 45 & 45 & 90 & 46 & 61 & 107 & natürlicher \\
\hline VII & 77 & 70 & 15 & 8,0 & 60 & 75 & 135 & 56 & 78 & 134 & $\begin{array}{l}\text { Chloridspitze im } \\
\text { Rohwasser }\end{array}$ \\
\hline XI & 38,5 & 70 & 10 & 5,2 & 20 & 90 & 110 & 42 & 116 & 158 & Boraxzusatz \\
\hline VII & 192 & 70 & 8 & 3 & 180 & 180 & 360 & 291 & 146 & 437 & $\begin{array}{l}\text { natürlicher } \\
\text { Chloridspitze im } \\
\text { Rohwasser }\end{array}$ \\
\hline
\end{tabular}

enthaltszeit» in den Belüftungskammern sich als Quotient aus Kammervolumen und gesamtem Wasserdurchsatz (RW + RS) und bei den Nachklärbecken aus dem Volumen des mutmasslich durchflossenen Absetzraumes und dem Rohwasserdurchsatz berechnet. Die schlechte Übereinstimmung zwischen theoretischer und «wirklicher» Durchflusszeit schreiben wir Kurzschlußströmungen in den Becken zu, die hauptsächlich wegen der geringen Distanzen zwischen Beckenzufluss und -abfluss relativ leicht möglich waren, wie dies zahlreiche Färbeversuche zeigten. Durch bauliche Massnahmen wurde nach Möglichkeit versucht, die Verhältnisse zu verbessern. Man muss jedoch bei allen kleineren Anlagen offenbar in Kauf nehmen, dass die wirklichen Aufenthaltszeiten in den verschiedenen Becken oft beträchtlich kürzer sein werden, als dies aus der Berechnung hervorgeht.

In den Tabellen Io und I4 haben wir die theoretischen Aufenthaltszeiten eingetragen, um die spätern Vergleiche mit ausländischen Anlagen durchführen zu können. 
Auf Grund der gemessenen Durchflusszeiten wurden der Beginn und das Ende der Probenahmeperioden in Rohwasser und Abfluss zeitlich so gestaffelt, dass die erhobenen Wasserproben miteinander übereinstimmten. Als Kontrolle für die Vergleichbarkeit von Rohwasser- und Abflußsammelproben diente der Cbloridgebalt, da dieser an den Umsetzungen in der Anlage nicht beteiligt ist. Wir haben in Tabelle 6 von drei Versuchsabschnitten aus Serien mit konstanter bzw. wechselnder Beschickung die Chloridkonzentrationen korrespondierender Sammelproben statistisch verglichen, um zu zeigen, dass die verwendeten Probenahmemethoden zutreffende Resultate erzielten. Die Differenz im Chloridgehalt der Sammelproben von Anlagenzu- und -abfluss ist in keinem der drei Beispiele statistisch gesichert. Damit ist auch gezeigt, dass mit unserer Definition der effektiven Durchflusszeit durch unsere Becken keine groben Fehler begangen werden.

Tabelle 6

Vergleicb der Cbloridgebalte im Robwasser und Anlagenabfluss bei Tagesmittel-Probenabmen

\begin{tabular}{|c|c|c|c|c|c|c|}
\hline \multirow{3}{*}{$\begin{array}{l}\text { Versuchs- } \\
\text { abschnitt }\end{array}$} & \multirow{3}{*}{ Beschickung } & \multicolumn{5}{|c|}{$\begin{array}{c}\text { Chloridgehalt der Tagesmittelproben, } \\
\text { Abschnittmittel }\end{array}$} \\
\hline & & \multicolumn{2}{|c|}{ Rohwasser } & \multicolumn{2}{|c|}{ Abfluss NKB } & \multirow{2}{*}{$\begin{array}{c}\text { Unterschied } \\
\text { zwischen } \\
\text { Rohwasser } \\
\text { und Abfluss } \\
\text { mg/1 }\end{array}$} \\
\hline & & $\mathrm{mg} / \mathrm{l}$ & $\begin{array}{c}\text { Streuung } \\
\mathrm{mg} / 1\end{array} \mid$ & $\mathrm{mg} / \mathrm{l}$ & $\left|\begin{array}{c}\text { Streuung } \\
\mathrm{mg} / \mathrm{l}\end{array}\right|$ & \\
\hline$V_{2}$ & konstant & 55 & $\pm 10,1$ & 53 & \pm 9 & 2 \\
\hline$V_{5}$ & wechselnd 4:1 & 47 & $\pm 11,5$ & 49 & $\pm 9,6$ & -2 \\
\hline$V_{0}$ & wechselnd $5: 1$ & 56 & $\pm 10,5$ & 55 & $\pm 11,0$ & 1 \\
\hline
\end{tabular}

\section{UNTERSUCHUNGSMETHODEN}

Je nach Problemstellung in den einzelnen Versuchsserien wurden mehr oder weniger weitgehende Beobachtungen und Messungen an den Versuchsanlagen vorgenommen. Der im allgemeinen sehr grosse Zeitaufwand für die eingehende Kontrolle mehrerer gleichzeitig laufender Experimente bedingte häufig eine Beschränkung der Untersuchungen auf das gemäss Fragestellung absolut notwendige Minimum.

Wir unterscheiden betriebstecbnische Messungen und Beobacbtungen sowie Methoden zur Messung der Reinigungsleistung der Versuchsanlagen. 


\section{a) Betriebstechnische Untersuchungen}

Wir fassen darunter die Beobachtungen zusammen, die einerseits notwendig sind, um eine Versuchsanlage gemäss den im Versuchsprogramm vorgeschriebenen Bedingungen zu führen, und anderseits das Verhalten einer Anlage im Laufe längerer Perioden zu kontrollieren. Die wichtigsten Beobachtungen und Methoden sind in Tabelle 7 zusammengefasst. Die

Tabelle 7

Betriebstecbniscbe Untersuchungen

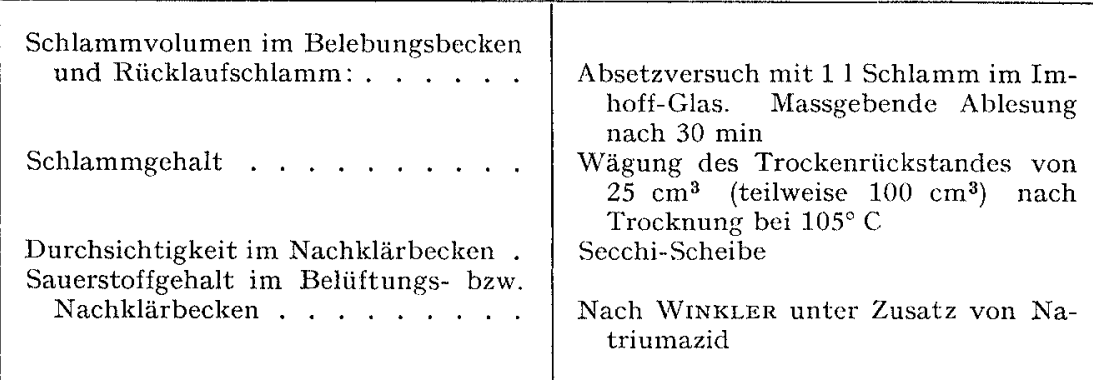

betriebstechnischen Untersuchungen erfolgten meistens täglich oder dann in kurzen Abständen, und zwar auch ausserhalb der eigentlichen Analysenperioden, so dass die Kontinuität der Versuchsbedingungen während der ganzen Versuchsdauer gewährleistet war.

\section{b) Prüfung der Reinigungsleistung einer Anlage}

Wir fassen unter diesem Titel alle Untersuchungen zusammen, die am rohen und gereinigten Abwasser angestellt wurden. Je nach Versuchsprogramm gestalteten sich die Analysen mehr oder weniger umfangreich. In zahlreichen Versuchen prüften wir beispielsweise in erster Linie die Stickstoffelimination und legten dabei auf andere analytische Kennzahlen des Abwassers weniger Wert. Dagegen wurde speziell zu Anfang der Experimente das Augenmerk mehr auf allgemeine Masszahlen für die organische Verunreinigung der Abwässer geworfen $\left(\mathrm{Bsb}_{5}, \mathrm{KMnO}_{4^{-}}\right.$ Verbrauch).

Die verwendete Analysenmethodik ist stichwortartig in Tabelle 8 aufgeführt. Zahlreiche Methoden wurden im Laufe der Jahre verbessert oder durch andere ersetzt, um die Präzision der Messungen zu erhöhen. Spe- 


\begin{tabular}{|c|c|}
\hline . . . . & $\begin{array}{l}\text { Potentiometrisch mit Glaselektrode. Alle Werte be- } \\
\text { zogen auf } 20^{\circ} \mathrm{C}\end{array}$ \\
\hline Leitfähigkeit . & $\begin{array}{l}\text { Elektrische Widerstandsmessung mit Philippsgerät } \\
\text { "Philoskop». Alle Werte bezogen auf } 20^{\circ} \mathrm{C}\end{array}$ \\
\hline Schwebestoffe & $\begin{array}{l}\text { Gooch-Tiegelmethode mit Zellulosematte oder als } \\
\text { Differenz des Trockenrückstandes einer unfiltrier- } \\
\text { ten und steril filtrierten Probe }\end{array}$ \\
\hline Trockenr ückstand & Nach Eindampfen von $100 \mathrm{~cm}^{3}$ Wasser bei $105^{\circ} \mathrm{C}$ \\
\hline Glühverlust & Glühen des Trockenrückstandes bei $600^{\circ} \mathrm{C}$ \\
\hline Oxydierbarkeit & Nach KubEL-ThremanN (Standard-Methods 1947) \\
\hline $\mathrm{Bsb}_{5}$ & $\begin{array}{l}\text { Verdünnungsmethode mit zehrungsfreiem Leitungs- } \\
\text { wasser ohne Salzzusatz, Bebrütung } 5 \text { Tage bei } 20^{\circ} \mathrm{C}\end{array}$ \\
\hline Organischer Stickstoff & Nach KJELDAHL, abzüglich $\mathrm{NH}_{4}$-Stickstoff \\
\hline Ammonium . . . & $\begin{array}{l}\text { Kolorimetrisch nach NESSLER oder titrimetrisch nach } \\
\text { Destillation mit Boraxpuffer pH 9,0 }\end{array}$ \\
\hline Nitrat & Nach ScherINGA (mit Sulfosalizylsäure) \\
\hline Nitrit. & Nach Bujwid (mit Indol) \\
\hline Aminosäure-N & $\begin{array}{l}\text { Nach VAN SLYKE und Mitarbeitern (1941) (im } \\
\text { Schlamm: nach Hydrolyse desselben in 20prozenti- } \\
\text { ger } \mathrm{H}_{2} \mathrm{SO}_{4} \text { während } 18 \mathrm{~h} \text { bei Siedetemperatur) }\end{array}$ \\
\hline $\begin{array}{l}\text { Organischer } \\
\text { Kohlenstoff }\end{array}$ & \\
\hline Konlenstott & $\begin{array}{l}\text { Nach VAN SLYKE und Mitarbeitern (1940): Eindamp- } \\
\text { fen der Wasserproben bei pH } 10 \text { und Ansäuern des } \\
\text { Rückstandes unmittelbar vor Analyse }\end{array}$ \\
\hline Kalium (im Schlamm) & Flammenspektrographisch im Kjeldahl-Aufschluss \\
\hline Chloride & Nach Mohr \\
\hline Schlammatmung & $\begin{array}{l}\text { Mikromanometrisch bei } 25^{\circ} \mathrm{C} \text { und } \mathrm{pH} 7,5-7,6 \text { nach } \\
\text { einmaliger Waschung des Schlammes in Leitungs- } \\
\text { wasser }\end{array}$ \\
\hline Saprophytische Bakterien & Verdünnungsmethode. Agar $22^{\circ} \mathrm{C}$. Zählung nach $120 \mathrm{~h}$ \\
\hline Bacterium coli & $\begin{array}{l}\text { Verdünnungsmethode. Endoagar } 37^{\circ} \mathrm{C} \text {. Zählung nach } \\
\quad 24 \mathrm{~h}\end{array}$ \\
\hline Phosphat & $\begin{array}{l}\text { "Molybdänblau »-Methode (im zentrifugierten Wasser } \\
\text { bestimmt) }\end{array}$ \\
\hline Gesamtphosphor & $\begin{array}{l}\text { Phosphat in der nicht zentrifugierten, aber veraschten } \\
\text { Probe bestimmt }\end{array}$ \\
\hline
\end{tabular}

zielle Schwierigkeiten waren stets bei der Bestimmung der Schwebestoffe $\mathrm{zu}$ überwinden, so dass die in Tabelle II aufgeführten Ziffern mit etwelchen Vorbehalten zu versehen sind.

Wir möchten grundsätzlich nochmals festhalten, dass die vorstehend geschilderten Untersuchungen auf keinen Fall als unentbehrlich für die praktische Führung einer Reinigungsanlage (Belebtschlamm- oder Tropfkörperverfahren) betrachtet werden dürfen. Die grosse Zahl der von uns vorgenommenen Beobachtungen diente den experimentellen Zielen. Über die zur Betriebsführung notwendigen Untersuchungen werden wir uns dagegen in einem besonderen Abschnitt (III, A I) verbreiten. 


\section{ZWEITER TEIL}

\section{DURCHGEFÜHRTE VERSUCHE, BETRIEBSBEOBACHTUNGEN} UND ANALYSENERGEBNISSE

Wir haben die Messungen und Analysen - soweit sie für Aussenstehende von Interesse sein könnten - von allen Versuchen, die wir im III. Teil besprechen möchten, in den Tabellen 9 bis 17 zusammengefasst.

Zu ihrer Erläuterung geben wir in kurzen Zügen eine summarische Darstellung der den einzelnen Versuchsserien zugrunde liegenden Fragestellungen.

\section{A. Versuche mit dem Belebtschlammverfahren}

Nachdem auf Grund von Versuchsserien früherer Jahre (die hier nicht weiter behandelt werden) feststand, dass das Belebtschlammverfahren günstige Ergebnisse auch bei hohen Belastungen zeitigen kann, wurden in Serie V systematisch verschiedene Belastungsstufen untersucht. Die aus diesem Experiment gewonnenen Erfahrungen waren jedoch äusserst beschränkt, da sie sich auf die Zeit grösster Lebensmittelknappheit bezogen, während der das städtische $A$ bwasser nur eine geringe Konzentration aufwies. Im Laufe späterer Versuche bei ähnlichen Raumbelastungen (Serien IX bis XIII) konnten die Erfahrungen mit Abwasser normaler Konzentration ergänzt werden. Eine spezielle Untersuchung über die zweistufige, biologische Abwasserreinigung mittels hintereinandergeschalteter Belebtschlamm- und Tropfkörperanlage erfolgte in den Serien VI und VII. Wir werden jedoch lediglich die Ergebnisse der Belebtschlammstufe darstellen. In den Serien VI, VII und IX wurde jeweils als absoluter Vergleichsmassstab für die Versuche mit hochbelasteten Anlagen eine mit konventioneller Belastung betriebene Vollreinigungsanlage geprüft.

$\mathrm{Zu}$ Beginn der Arbeiten herrschte die Auffassung, ein reibungsloser Betrieb einer hocbbelasteten Belebtschlammanlage sei nur möglich unter Anwendung eines Asbestzusatzes in die Belüftungsbecken. Im Laufe der ersten Versuche hatte sich die Praxis einer Asbestbeimischung von $1 \mathrm{mg}$ auf $1 \mathrm{l}$ Rohmasser ausgebildet. Alle Versuchsabschnitte mit der Bezeichnung « a» in den Tabellen 9 bis I 3 sind mit diesem Asbestzusatz ausgeführt worden. Ausnahme: in Serie $\mathrm{Xa}_{2}^{20}$ erfolgte eine Asbestdosis von $20 \mathrm{mg} / \mathrm{l}$. Im Gegensatz dazu sind alle Versuchsabschnitte, bei denen keine Asbestzusätze verwendet wurden, mit «o» bezeichnet. Schon bald nach Durchführung der Serie V 
erhob sich das Bedürfnis, die Frage der Bedeutung des Asbestes genauer abzuklären. Die Serie X planten wir speziell im Hinblick auf dieses Problem und führten sie in zwei Belastungsstufen durch. Gleichzeitig verwirklichten wir in dieser Serie den Wunsch nach möglichst weitgehender Untersuchung sowohl der Stickstoffbilanz als auch des Abbaues organischer Verbindungen im allgemeinen.

Serie XII diente speziell der Abklärung des Einflusses geringer Mengen eines phenolhaltigen industriellen Abwassers auf eine Belebtschlammanlage. Die verschiedenen Abschnitte dieser Serie betrachten wir zusammengefasst. Da es sich neben der Verfolgung des Phenols bei der Abwasserreinigung im übrigen nur um die Kontrolle des Verhaltens der Anlage und die vergleichsweise Prüfung der Abflussqualität in verschiedenen Betriebsphasen handelte, wurden nur Einzelproben untersucht, die keine bilanzmässigen Schlüsse gestatten. Das gleiche gilt für die Versuche in Serie XIII, in denen speziell das Verhalten einer mittelmässig belasteten Anlage gegenüber sehr schwach vorgeklärtem Rohwasser studiert wurde. Gleichzeitig erfolgte in dieser Serie auch die vergleichsmässige Prüfung zweier hochbelasteter Anlagen.

\section{B. Versuche mit dem künstlich belüfteten Tropfkörper}

Die ersten Experimente (Serie V) wurden im Sinne von Vergleichsversuchen mit andern Tropfkörpersystemen (hier nicht näher besprochen) und hochbelasteten Belebtschlammanlagen mit dem Ziele durchgefuihrt, einen allgemeinen Überblick über die Leistungsfähigkeit des künstlich belüfteten Tropfkörpers bei verschiedenen Belastungen zu gewinnen. Wie wir dies bereits bei der entsprechenden Serie des Belebtschlammverfahrens schilderten, ergaben die Versuche nur beschränkte Informationen (sehr stark verdünntes $A$ bwasser). Bei günstigeren Verhältnissen verfolgten wir in Serie VIII bei zwei Belastungsstufen speziell die Elimination pon Stickstoffverbindungen. Die Serien IX und X dienten in erster Linie dazu, nochmals mittels eingehender Messungen die Abbauleistung des Tropfkörpers zu bestimmen, wobei wir in Serie X die Raumbelastung bis zur Höhe von I2 $\mathrm{m}^{3} / \mathrm{m}^{3} \cdot$ Tag steigerten.

Der vor Beginn der Serie VIII eingebaute neue Ventilator ermöglichte in den Versuchen Serie VIII bis X eine genauere Messung des Luftdurchsatzes durch den Körper, als dies in Serie V der Fall war. 
Versucbe mit Belebtscblammperfabren, Versucbseinricbtungen

$($ StA $=$ Kläranlage der Stadt Zürich; $\mathrm{S}=$ Schöpfrad; $\mathrm{ZP}=$ Zentrifugalpumpe, Bauart Egger $)$

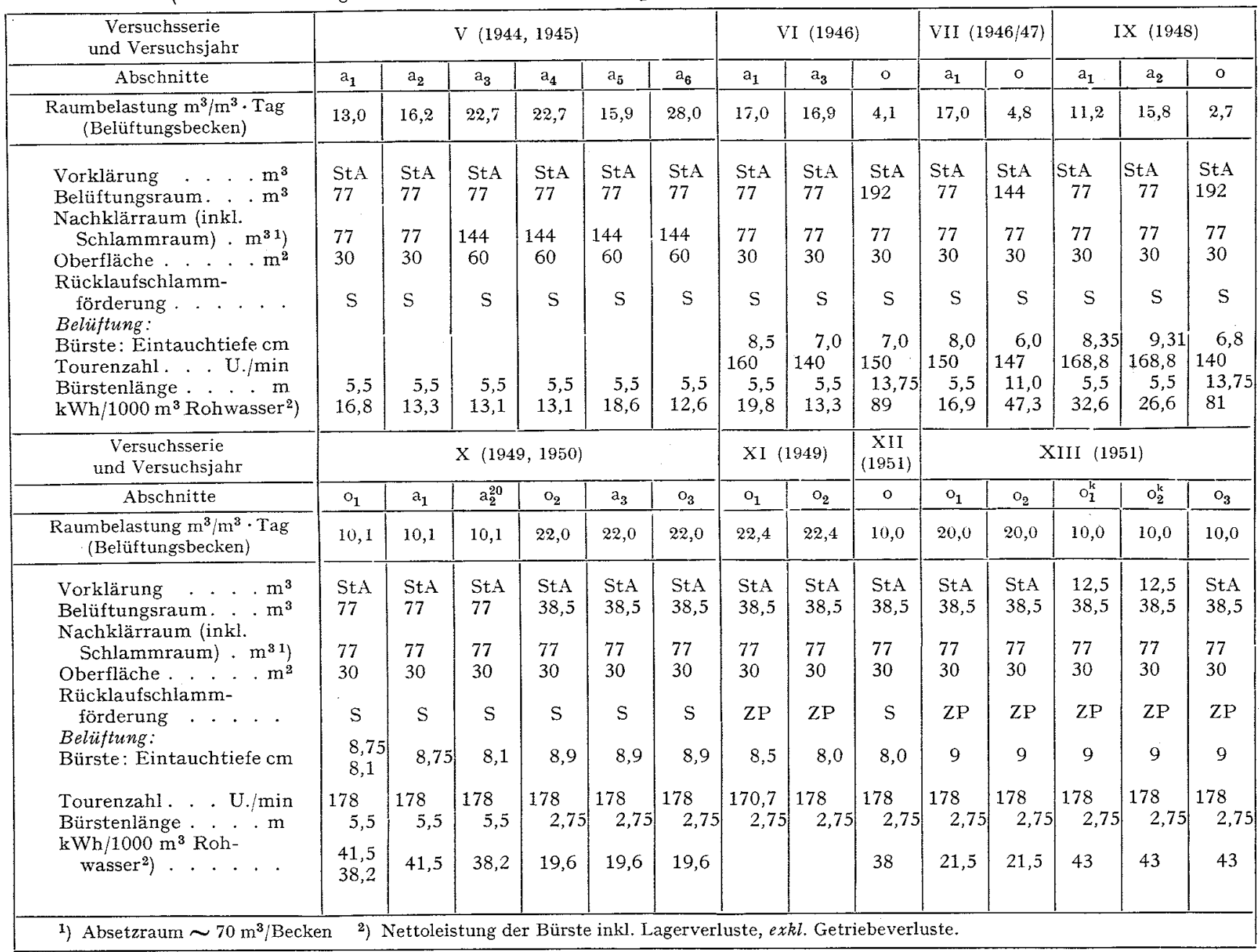

1) Absetzraum $\sim 70 \mathrm{~m}^{3} /$ Becken ${ }^{2}$ ) Nettoleistung der Bürste inkl. Lagerverluste, exkl. Getriebeverluste. 
Tabelle 10

Versucbe mit Belebtscblammperfabren. Effektipe Betriebsdaten (Abschnittsmittel)

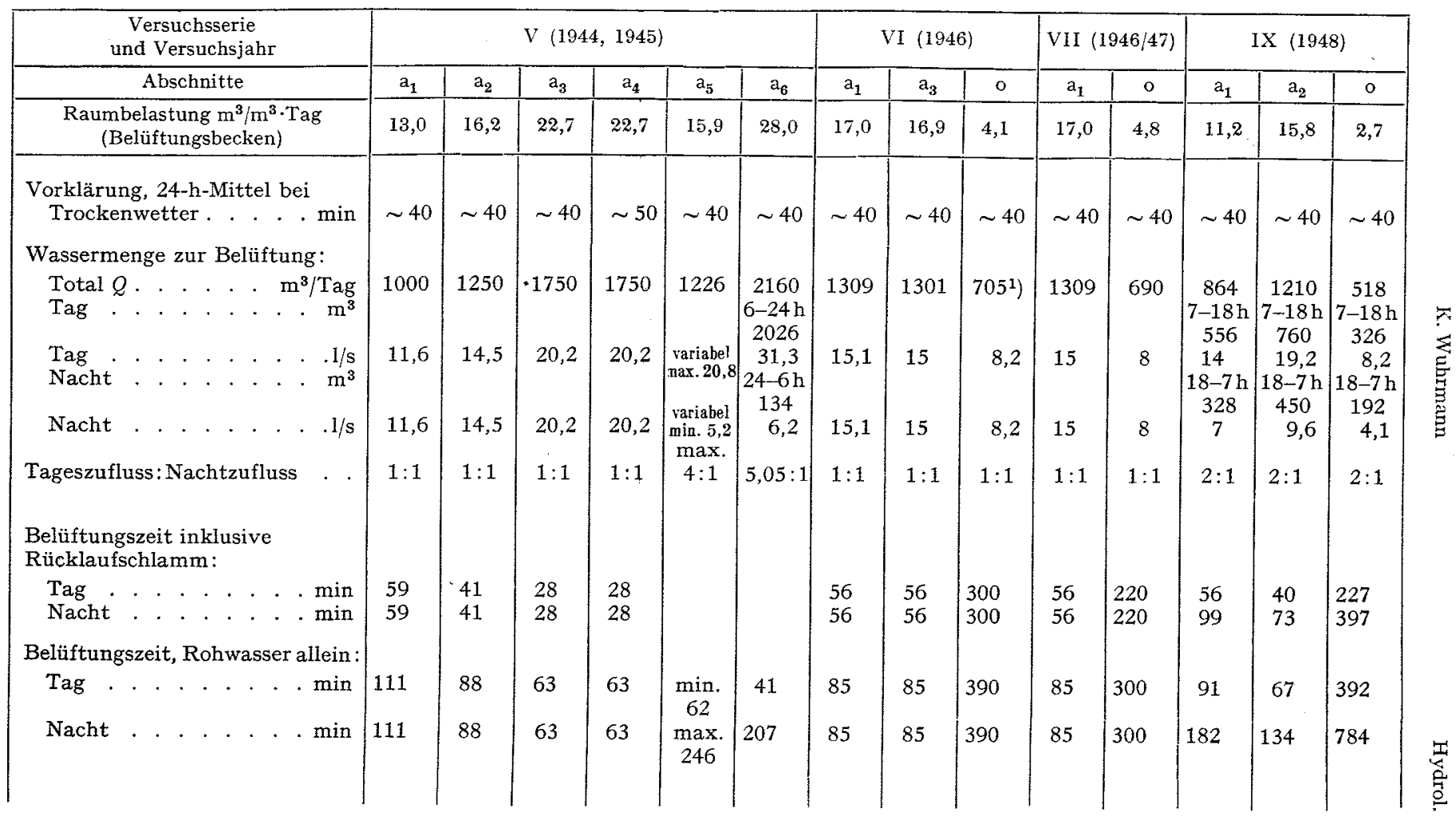




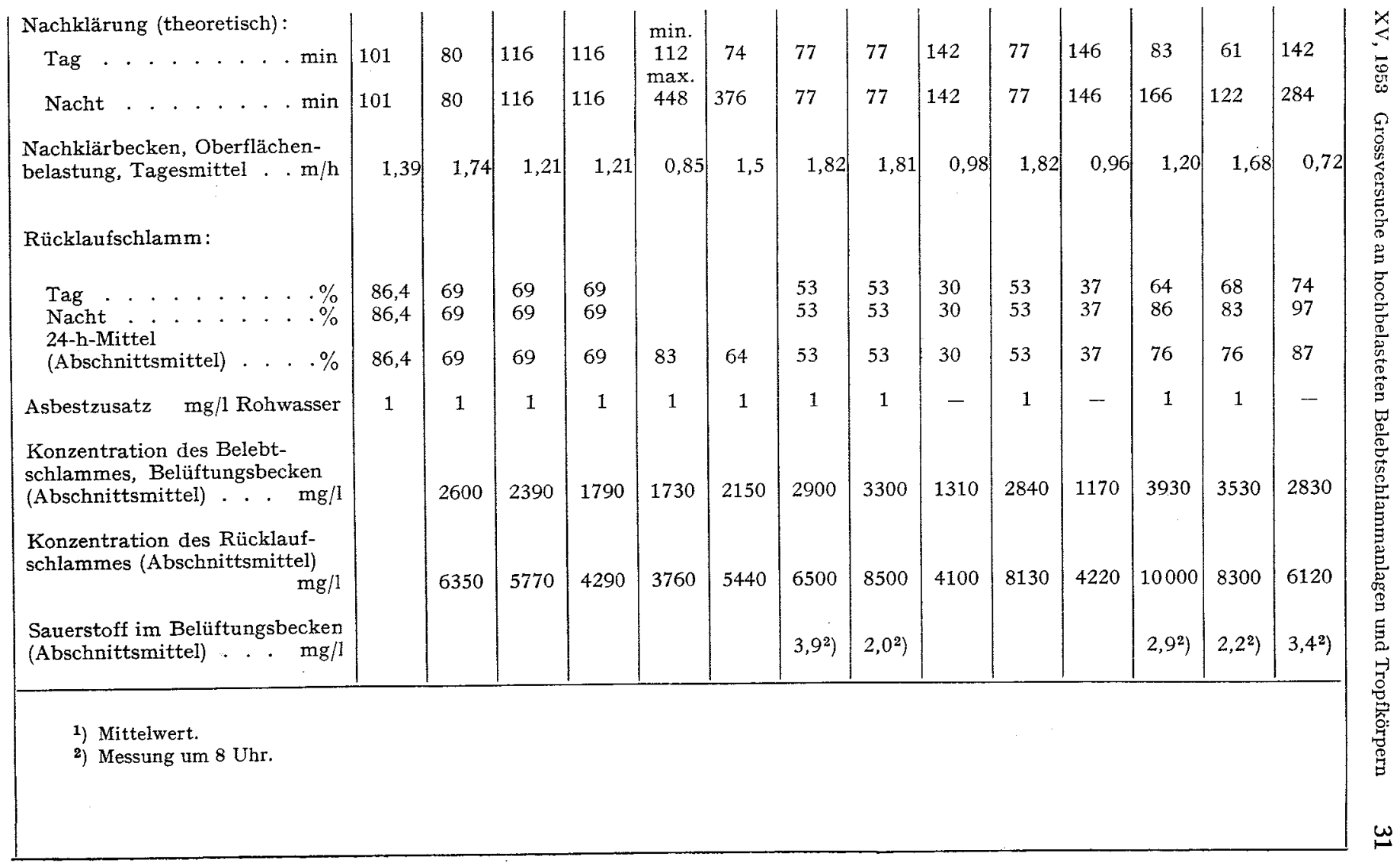


Tabelle 10 (Fortsetzung)

\begin{tabular}{|c|c|c|c|c|c|c|c|c|c|c|c|c|c|c|}
\hline \multirow{2}{*}{$\begin{array}{c}\begin{array}{c}\text { Versuchsserie } \\
\text { und Versuchsjahr }\end{array} \\
\text { Abschnitte } \\
\end{array}$} & \multicolumn{6}{|c|}{$\mathrm{X}(1949,1950)$} & \multicolumn{2}{|c|}{ XI (1949) } & $\begin{array}{c}\mathrm{XII} \\
(1951)\end{array}$ & \multicolumn{5}{|c|}{ XIII (1951) } \\
\hline & $\mathrm{o}_{1}$ & $a_{1}$ & $a_{2}^{20}$ & $o_{2}$ & $a_{3}$ & $\mathrm{O}_{3}$ & $o_{1}$ & $\mathrm{O}_{2}$ & 0 & $o_{1}$ & $\mathrm{O}_{2}$ & $0_{1}^{k}$ & $o_{2}^{k}$ & $\mathrm{O}_{3}$ \\
\hline $\begin{array}{c}\text { Raumbelastung } \mathrm{m}^{3} / \mathrm{m}^{3} \cdot \mathrm{Tag} \\
\text { (Belüftungsbecken) }\end{array}$ & 10,1 & 10,1 & 10,1 & 22,0 & 22,0 & 22,0 & 22,4 & 22,4 & 10,0 & 20,0 & 20,0 & 10,0 & 10,0 & 10,0 \\
\hline $\begin{array}{l}\text { Vorklärung, 24-h-Mittel bei } \\
\text { Trockenwetter... . min } \\
\text { Wassermenge zur Belüftung: }\end{array}$ & $\sim 40$ & $\sim 40$ & $\sim 40$ & $\sim 40$ & $\sim 40$ & $\sim 40$ & $\sim 40$ & $\sim 40$ & $\sim 40$ & $\sim 45$ & $\sim 45$ & $\sim 20$ & $\sim 10$ & $\sim 45$ \\
\hline 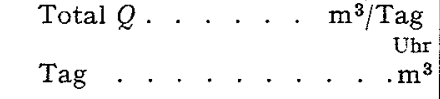 & $\begin{array}{c}780 \\
8-16.30 \\
390\end{array}$ & $\begin{array}{c}780 \\
8-16.30 \\
390\end{array}$ & $\begin{array}{c}780 \\
8-16.30 \\
390\end{array}$ & $\begin{array}{c}846 \\
8-16.30 \\
422\end{array}$ & $\begin{array}{c}846 \\
8-16.30 \\
422\end{array}$ & $\mid \begin{array}{c}846 \\
8-16.30 \\
422\end{array}$ & 864 & 864 & 385 & 780 & 780 & 385 & 385 & 385 \\
\hline 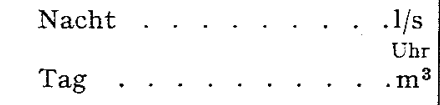 & $\begin{array}{c}12,7 \\
16.30-8 \\
390\end{array}$ & $\begin{array}{c}12,7 \\
16.30-8 \\
390\end{array}$ & $\begin{array}{c}12,7 \\
16.30-8 \\
390\end{array}$ & $\begin{array}{c}13,8 \\
16.3 C-8 \\
424\end{array}$ & $\begin{array}{c}13,8 \\
16.30-8 \\
424\end{array}$ & $\begin{array}{c}13,8 \\
16.30-8 \\
424\end{array}$ & 10 & 10 & 4,5 & 9 & 9 & 4,5 & 4,5 & 4,5 \\
\hline Nacht $\cdot \cdot \cdot \cdot \cdot \cdot \cdot \cdot .1 / \mathrm{s}$ & 7,0 & 7,0 & 7,0 & 7,6 & 7,6 & 7,6 & 10 & 10 & 4,5 & 9 & 9 & 4,5 & 4,5 & 4,5 \\
\hline $\begin{array}{l}\text { Tageszufluss: Nachtzufluss . . } \\
\text { Belüftungszeit inklusive } \\
\text { Rücklaufschlamm: }\end{array}$ & $1,83: 1$ & $1,83: 1$ & $1,83: 1$ & $1,83: 1$ & $1,83: 1$ & $1,83: 1$ & $1: 1$ & $1: 1$ & $1: 1$ & $1: 1$ & $1: 1$ & $1: 1$ & $1: 1$ & $1: 1$ \\
\hline Tag . . . . . . . $\cdot \min$ & $\begin{array}{l}61 \\
64\end{array}$ & 60 & 62 & 34 & 34 & 34 & 42 & 42 & 75 & 43 & 43 & 75 & 75 & 75 \\
\hline $\begin{array}{l}\text { Nacht } . . . . . . ~ \\
\text { Belüftungszeit, Rohwasser allein: }\end{array}$ & $\begin{array}{r}95 \\
103\end{array}$ & 96 & 100 & 50 & 50 & 50 & 42 & 42 & 75 & 43 & 43 & 75 & 75 & 75 \\
\hline Tag . . . . . . . $\min$ & $\begin{array}{l}101 \\
101\end{array}$ & 101 & 101 & 46 & 46 & 46 & 64 & 64 & 144 & 71 & 71 & 144 & 144 & 144 \\
\hline Nacht . . . . . . . min & $\begin{array}{l}184 \\
184\end{array}$ & 184 & 184 & 84 & 84 & 84 & 64 & 64 & 144 & 71 & 71 & 144 & 144 & 144 \\
\hline
\end{tabular}




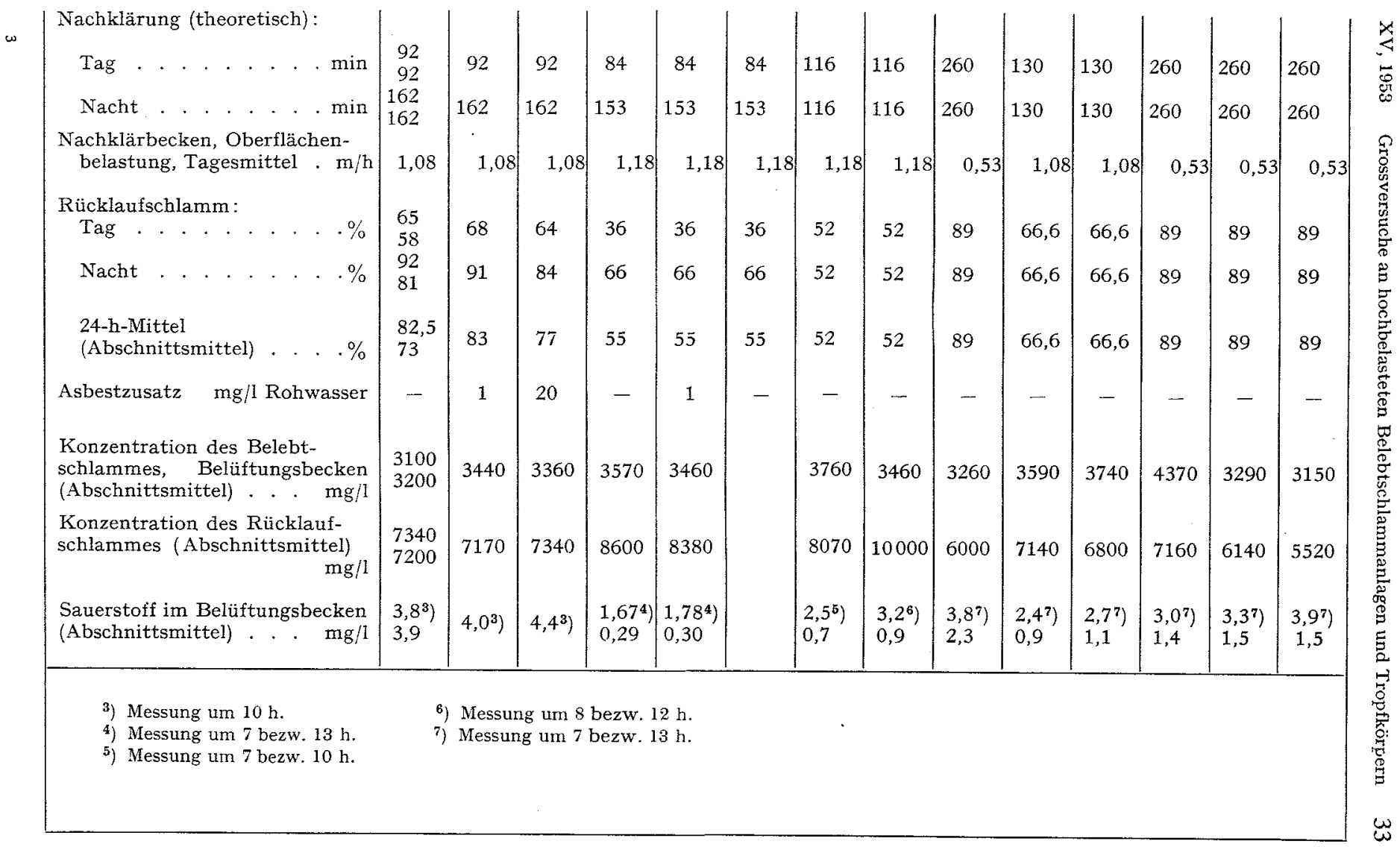


Versucbe mit Belebtscblammverfabren, Analysenresultate (Abschnittsmittel)

( $\mathrm{RW}=$ Rohwasser (vorgeklärt); $\mathrm{A}=$ Nachklärbecken-Abfluss)

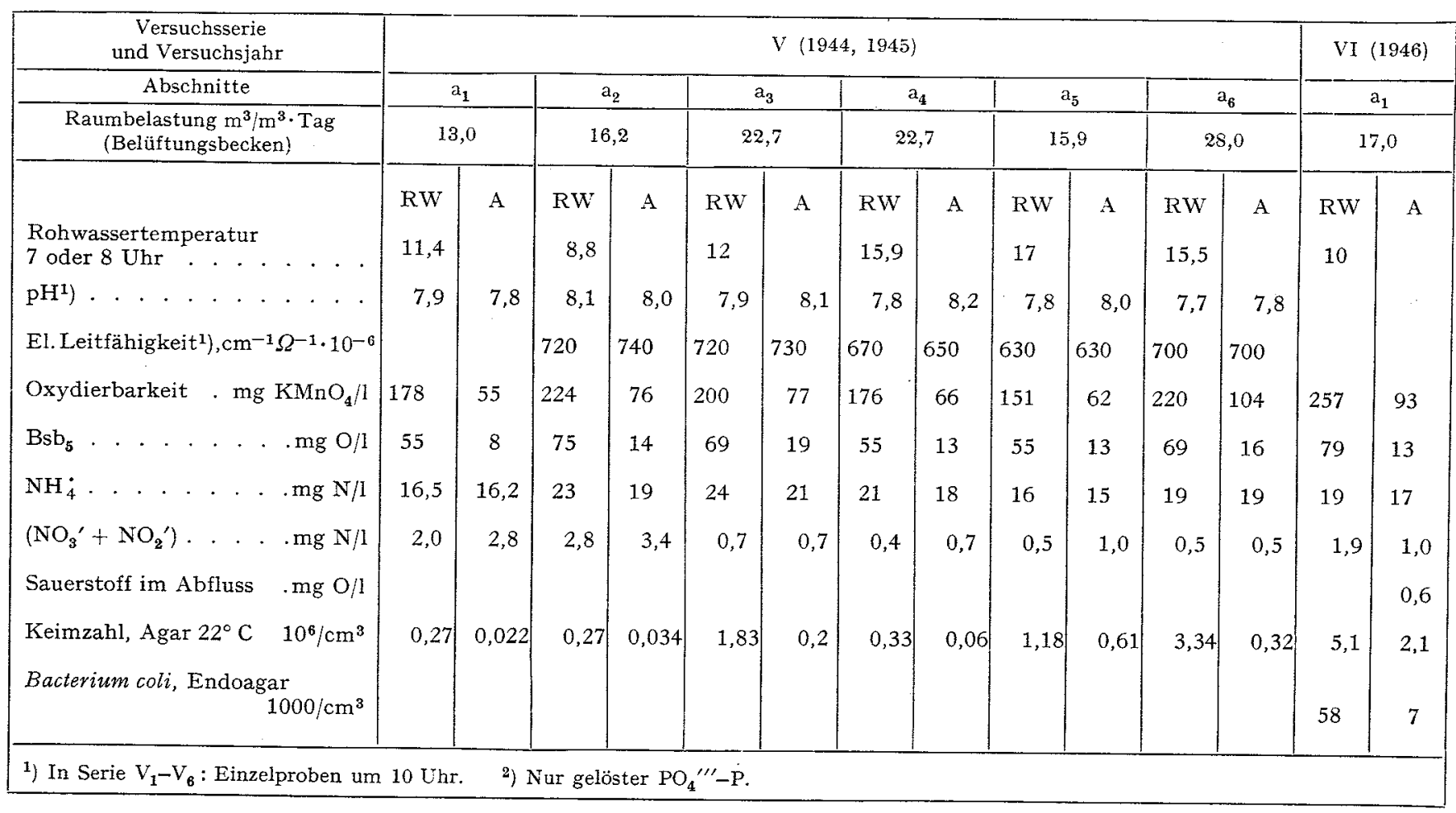


Tabelle 11 (Fortsetzung)

\begin{tabular}{|c|c|c|c|c|c|c|c|c|c|c|c|c|c|c|}
\hline $\begin{array}{l}\text { Versuchsserie } \\
\text { und Versuchsjahr }\end{array}$ & \multicolumn{4}{|c|}{ VI $(1946)$} & \multicolumn{4}{|c|}{ VII $(1946 / 47)$} & \multicolumn{6}{|c|}{$\mathrm{IX}(1948)$} \\
\hline Abschnitte & \multicolumn{2}{|c|}{$a_{3}$} & \multicolumn{2}{|c|}{ o } & \multicolumn{2}{|c|}{$a_{1}$} & \multicolumn{2}{|c|}{0} & \multicolumn{2}{|c|}{$a_{1}$} & \multicolumn{2}{|c|}{$\mathrm{a}_{2}$} & \multicolumn{2}{|c|}{$\overrightarrow{\mathrm{o}}$} \\
\hline $\begin{array}{c}\text { Raumbelastung } \mathrm{m}^{3} / \mathrm{m}^{3} \cdot \text { Tag } \\
\text { (Belüftungsbecken) }\end{array}$ & \multicolumn{2}{|c|}{16,9} & \multicolumn{2}{|c|}{4,1} & \multicolumn{2}{|c|}{17,0} & \multicolumn{2}{|c|}{4,8} & \multicolumn{2}{|c|}{11,2} & \multicolumn{2}{|c|}{15,8} & \multicolumn{2}{|c|}{2,7} \\
\hline & RW & A & RW & A & RW & $\mathrm{A}$ & RW & $\mathrm{A}$ & RW & A & RW & A & RW & A \\
\hline 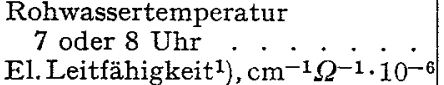 & 15 & & 16 & & 653 & 641 & 622 & 665 & 15,4 & & 15,8 & & 15,6 & \\
\hline $\begin{array}{lll}\text { Trockenrückstand . . . } & \mathrm{mg} / \mathrm{l} \\
\text { Glühverlust desselben. . } & \mathrm{mg} / \mathrm{l}\end{array}$ & & & & & & & & & $\begin{array}{l}543 \\
321\end{array}$ & $\begin{array}{l}408 \\
248\end{array}$ & $\begin{array}{l}610 \\
250\end{array}$ & $\begin{array}{l}430 \\
130\end{array}$ & $\begin{array}{l}543 \\
610 \\
321 \\
250\end{array}$ & $\begin{array}{l}435 \\
468 \\
238 \\
163\end{array}$ \\
\hline Schwebestoffe . . . . $\mathrm{mg} / 1$ & & & & & & & & & 61 & 10 & 97 & 13 & $\begin{array}{l}61 \\
97\end{array}$ & $\begin{array}{r}8 \\
11\end{array}$ \\
\hline Oxydierbarkeit . mg $\mathrm{KMnO}_{4} / 1$ & 263 & 95 & 256 & 74 & 345 & 107 & 343 & 115 & & & & & & \\
\hline $\mathrm{Bsb}_{5} \cdot \cdots \cdot \cdot \mathrm{mg} \mathrm{O} / \mathrm{l}$ & 72 & 15 & 78 & 8 & 69 & 13 & 72 & 13 & 123 & 13 & 150 & 26 & $\begin{array}{l}123 \\
150\end{array}$ & $\begin{array}{l}8 \\
8\end{array}$ \\
\hline Gesamtstickstoff . . . mg N/I & 32,6 & 20,9 & 32,7 & 16,4 & 25,0 & 19,4 & 25,8 & 21,8 & 23,6 & 12,9 & 23,7 & 13,7 & $\begin{array}{l}23,6 \\
23,7\end{array}$ & $\begin{array}{l}12,1 \\
14,9\end{array}$ \\
\hline Organischer Stickstoff .mg N/l & 8,3 & 0,4 & 8,3 & 0,6 & 7,8 & 3,4 & 8,0 & 3,0 & 10,9 & 2,4 & 7,2 & 1,6 & $\begin{array}{r}10,9 \\
7,2\end{array}$ & $\begin{array}{l}1,4 \\
0,7\end{array}$ \\
\hline $\mathrm{NH}_{4} \cdot . \cdot . \cdot . \cdot . \cdot \mathrm{mg} \mathrm{N} / 1$ & 22,8 & 19,3 & 22,8 & 7,1 & 15,5 & 15,1 & 15,1 & 14,8 & 12,3 & 9,5 & 16,0 & 11,7 & $\begin{array}{l}12,3 \\
16,0\end{array}$ & $\begin{array}{l}2,4 \\
3,5\end{array}$ \\
\hline$\left(\mathrm{NO}_{3}{ }^{\prime}+\mathrm{NO}_{2}^{\prime}\right) \cdot \ldots \cdot \cdot \mathrm{mg} \mathrm{N} / \mathrm{l}$ & 1,5 & 1,0 & 1,6 & 8,7 & 1,7 & 0,9 & 2,7 & 4,0 & 0,4 & 1,0 & 0,4 & 0,4 & $\begin{array}{l}0,4 \\
0,4\end{array}$ & $\begin{array}{r}8,3 \\
10,7\end{array}$ \\
\hline Gesamtphosphor. . . .mg P/I & & & & & $\left.1,5^{2}\right)$ & $\left.1,1^{2}\right)$ & $\left.1,4^{2}\right)$ & $\left.1,4^{2}\right)$ & $\left.1,13^{2}\right)$ & $\left.0,72^{2}\right)$ & 3,53 & 1,39 & 3,53 & 2,84 \\
\hline $\begin{array}{l}\text { Aminosäure-Stickstoff } . \mathrm{mg} \mathrm{N} / 1 \\
\text { Sauerstoff im Abfluss }\end{array}$ & & 10 & & 73. & & 156 & & 72 & 0,44 & 0,09 & & & $\begin{array}{l}1,13 \\
0,44\end{array}$ & $\begin{array}{l}1,01 \\
0,13\end{array}$ \\
\hline $\begin{array}{l}\text { Keimzahl, Agar } 22^{\circ} \mathrm{C} 10^{6} / \mathrm{cm}^{3} \\
\text { Bacterium coli, Endoagar }\end{array}$ & 5,1 & 2,1 & 5,1 & 1,9 & & & & & & & & & & \\
\hline $1000 / \mathrm{cm}^{3}$ & 84 & 6,7 & 71 & 8,7 & & & & & & & & & & \\
\hline
\end{tabular}


Tabelle 11 (Fortsetzung)

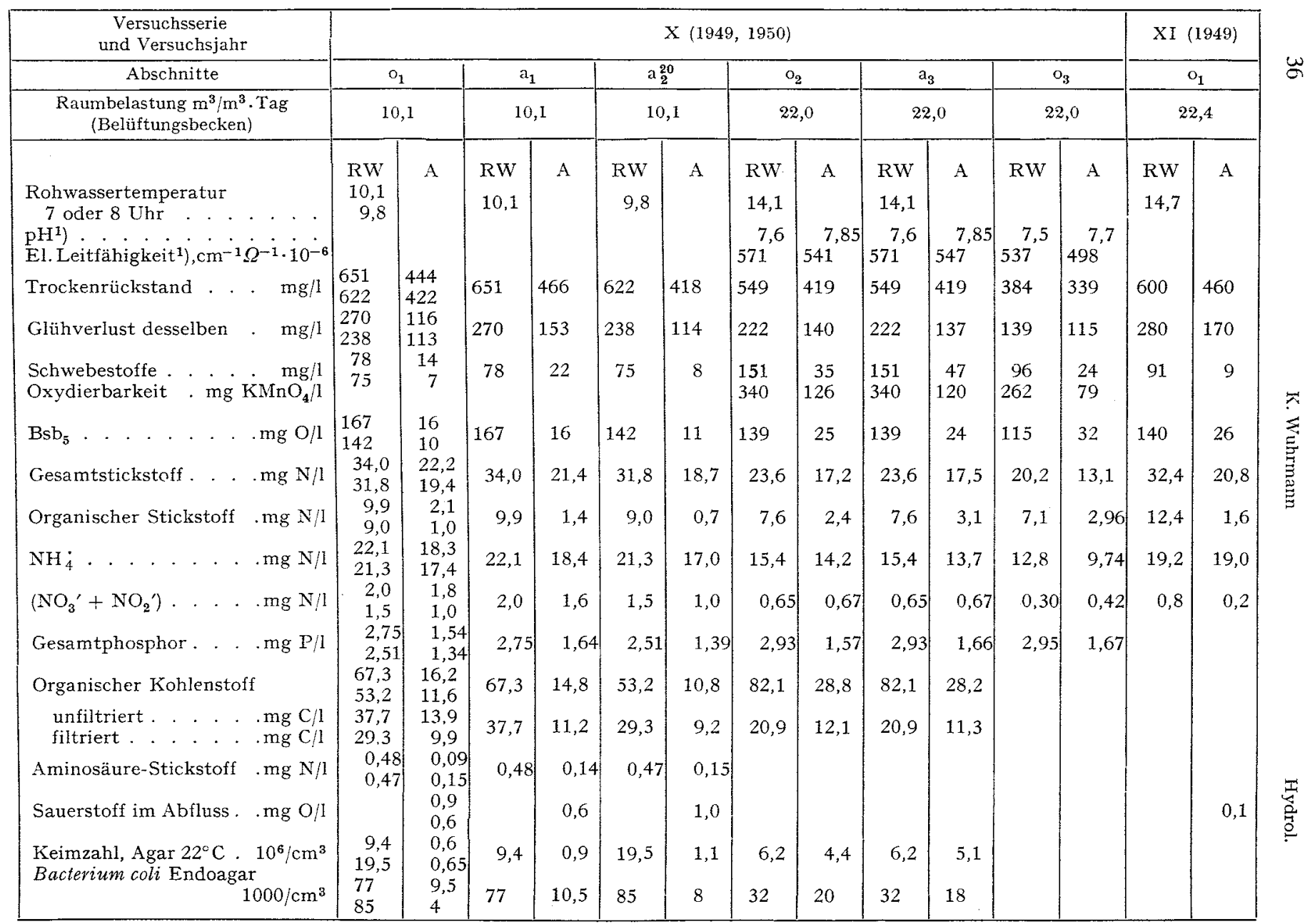


Tabelle 11 (Fortsetzung)

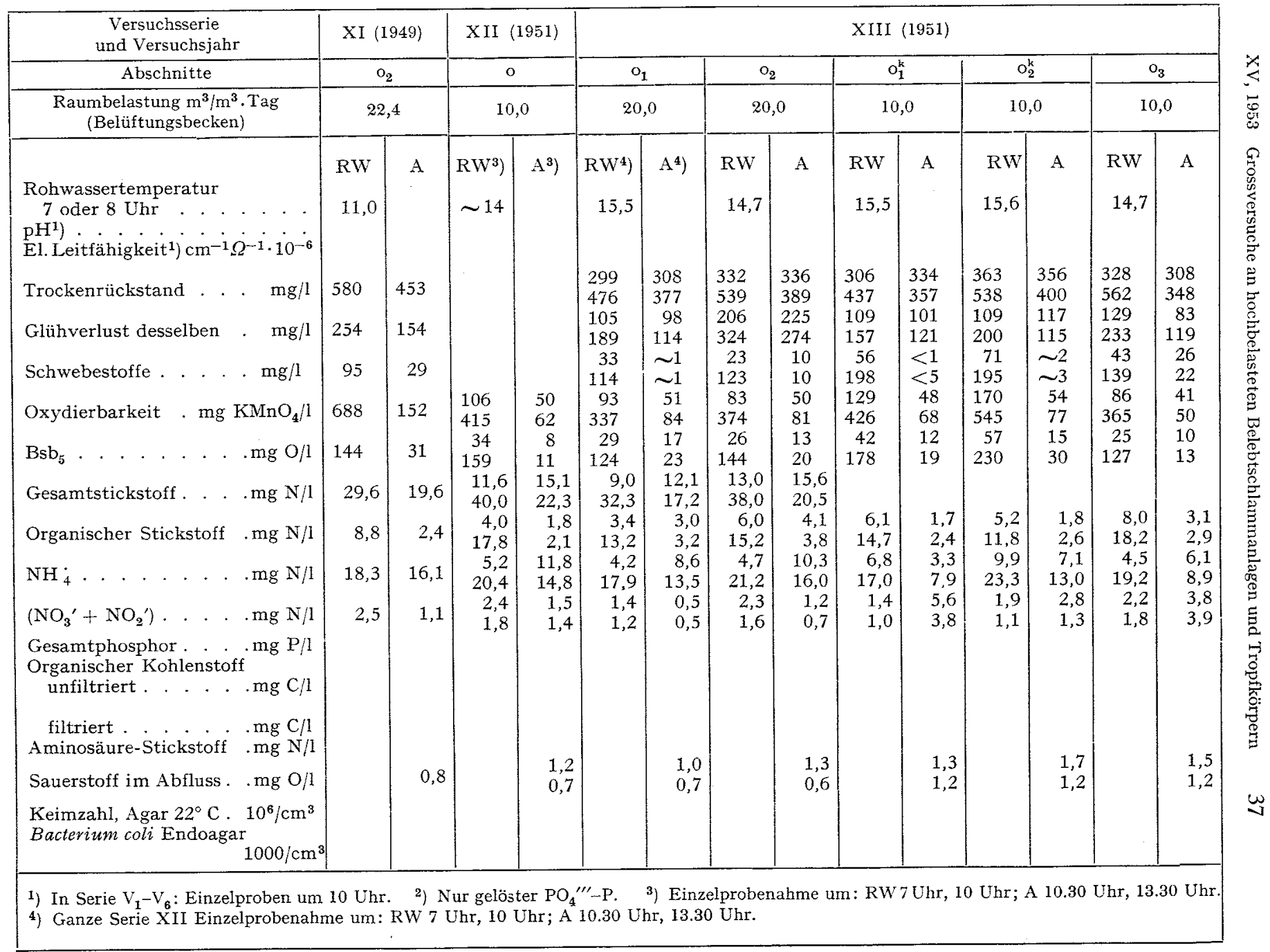


Tabelle 12

Versuche mit Belebtscblammperfabren, Raumbelastung, Abbauleistung, Reinigungseffekt (Abschnittsmittel)

(Für die Serien XII und XIII sind keine Berechnungen möglich, da nur Einzelproben analysiert wurden)

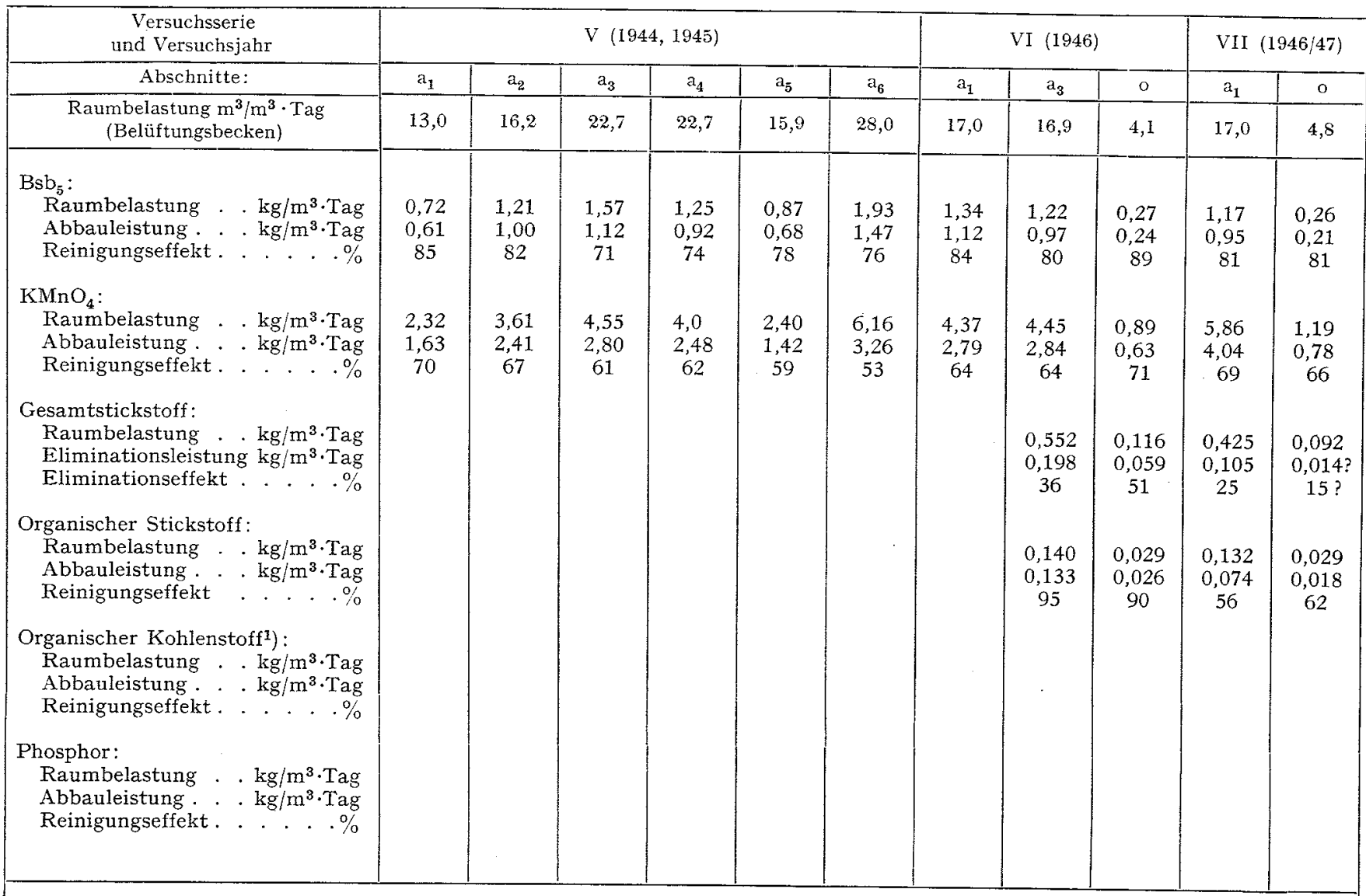

1) Analyse im unfiltrierten Wasser 


\begin{tabular}{|c|c|c|c|c|c|c|c|c|c|c|c|}
\hline $\begin{array}{l}\text { Versuchsserie } \\
\text { und Versuchsjahr }\end{array}$ & \multicolumn{3}{|c|}{ IX $(1948)$} & \multicolumn{6}{|c|}{$X(1949,1950)$} & \multicolumn{2}{|c|}{ XI $(1949)$} \\
\hline Abschnitte & $a_{1}$ & $\mathrm{a}_{2}$ & o & $o_{1}$ & $a_{1}$ & $\mathrm{a}_{2}^{20}$ & $\mathrm{o}_{2}$ & $a_{3}$ & $\mathrm{o}_{3}$ & $o_{1}$ & $\mathrm{o}_{2}$ \\
\hline $\begin{array}{l}\text { Raumbelastung } \mathrm{m}^{3} / \mathrm{m}^{3} \cdot \mathrm{Tag} \\
\text { (Belüftungsbecken) }\end{array}$ & 11,2 & 15,8 & 2,7 & 10,1 & 10,1 & 10,1 & 22,0 & 22,0 & 22,0 & 22,4 & 22,4 \\
\hline $\mathrm{Bsb}_{5}:$ & & & & & & & & & & & \\
\hline Raumbelastung $\cdot \mathrm{kg} / \mathrm{m}^{3} \cdot \mathrm{Tag}$ & 1,38 & 2,36 & 0,37 & $\begin{array}{l}1,69 \\
1,44\end{array}$ & $\begin{array}{c}1,69 \\
-\end{array}$ & $1, \overline{44}$ & 3,06 & 3,06 & 2,53 & 3,14 & 3,23 \\
\hline Abbauleistung . . $. \mathrm{kg} / \mathrm{m}^{3} \cdot \mathrm{Tag}$ & 1,23 & 1,95 & 0,35 & $\begin{array}{l}1,53 \\
1,34\end{array}$ & $\begin{array}{c}1,53 \\
-\end{array}$ & $1, \overline{27}$ & 2,51 & 2,53 & 1,83 & 2,56 & 2,54 \\
\hline Reinigungseffekt. . . . . .\% & 89 & 83 & 94 & $\begin{array}{l}91 \\
93\end{array}$ & $\begin{array}{l}91 \\
-\end{array}$ & $\overrightarrow{88}$ & 82 & 83 & 72 & 82 & 79 \\
\hline $\begin{array}{l}\mathrm{KMnO}_{4}: \\
\mathrm{Raumbelastung} \cdot . \mathrm{kg} / \mathrm{m}^{3} \cdot \mathrm{Tag} \\
\text { Abbauleistung.. } \cdot \mathrm{kg} / \mathrm{m}^{3} \cdot \mathrm{Tag} \\
\text { Reinigungseffelit. . . . . } \%\end{array}$ & & & & & & & $\begin{array}{r}7,48 \\
4,71 \\
63\end{array}$ & $\begin{array}{r}7,48 \\
4,84 \\
65\end{array}$ & $\begin{array}{r}5,76 \\
4,02 \\
70\end{array}$ & & $\begin{array}{r}5,82 \\
4,05 \\
70\end{array}$ \\
\hline $\begin{array}{l}\text { Gesamtstickstoff: } \\
\text { Raumbelastung } . . \mathrm{kg} / \mathrm{m}^{3} \cdot \text { Tag }\end{array}$ & 0,264 & 0,373 & 0,063 & $\begin{array}{l}0,35 \\
0,322\end{array}$ & $\begin{array}{l}0,35 \\
-\end{array}$ & $0, \overline{322}$ & 0,52 & 0,52 & 0,444 & 0,725 & 0,664 \\
\hline Eliminationsleistung $\mathrm{kg} / \mathrm{m}^{3} \cdot \mathrm{Tag}$ & 0,119 & 0,157 & 0,027 & $\begin{array}{l}0,12 \\
0,12\end{array}$ & $\begin{array}{l}0,13 \\
-\end{array}$ & $0, \overline{132}$ & 0,14 & 0,14 & 0,156 & 0,237 & 0,225 \\
\hline Eliminationseffekt . . . . \% & 45 & 42 & 43 & $\begin{array}{l}35 \\
37\end{array}$ & $\begin{array}{l}37 \\
-\end{array}$ & $\overline{41}$ & 27 & 27 & 35 & 33 & 34 \\
\hline $\begin{array}{l}\text { Organischer Stickstoff: } \\
\text { Raumbelastung } \cdot . \mathrm{kg} / \mathrm{m}^{3} \cdot \text { Tag }\end{array}$ & 0,122 & 0,113 & 0,024 & $\begin{array}{l}0,10 \\
0,091\end{array}$ & $\begin{array}{l}0,10 \\
-\end{array}$ & $0, \overline{0} 91$ & 0,167 & 0,167 & 0,156 & 0,276 & 0,197 \\
\hline Abbauleistung . . . $\mathrm{kg} / \mathrm{m}^{3} \cdot \mathrm{Tag}$ & 0,095 & 0,088 & 0,022 & $\begin{array}{l}0,077 \\
0,080\end{array}$ & 0,086 & $0, \overline{0} 83$ & 0,113 & 0,099 & 0,091 & 0,240 & 0,144 \\
\hline Reinigungseffekt. . . . $\%$ & 78 & 78 & 92 & $\begin{array}{l}77 \\
88\end{array}$ & $\begin{array}{l}86 \\
-\end{array}$ & $\overline{91}$ & 68 & 59 & 58 & 87 & 73 \\
\hline $\begin{array}{l}\left.\text { Organischer Kohlenstoff }{ }^{1}\right): \\
\text { Raumbelastung } \cdot . \mathrm{kg} / \mathrm{m}^{3} \cdot \text { Tag }\end{array}$ & & & & $\begin{array}{l}0,682 \\
0,539\end{array}$ & 0,682 & $0, \overline{539}$ & 1,805 & 1,805 & & & \\
\hline Abbauleistung . . $\cdot \mathrm{kg} / \mathrm{m}^{3} \cdot$ Tag & & & & $\begin{array}{l}0,517 \\
0,422\end{array}$ & $\begin{array}{c}0,532 \\
-\end{array}$ & $0, \overline{4} 29$ & 1,170 & 1,183 & & & \\
\hline Reinigungseffekt. . . . . .\% & & & & $\begin{array}{l}76 \\
78\end{array}$ & $\begin{array}{l}78 \\
-\end{array}$ & $\overline{79}$ & 65 & 65 & & & \\
\hline $\begin{array}{l}\text { Phosphor: } \\
\text { Raumbelastung } . \quad . \mathrm{kg} / \mathrm{m}^{3} \cdot \mathrm{Tag}\end{array}$ & & & & $\begin{array}{l}0,028 \\
0,025\end{array}$ & 0,028 & $\overline{0,025}$ & 0,0645 & 0,0645 & 0,065 & & \\
\hline Abbauleistung . . $\cdot \mathrm{kg} / \mathrm{m}^{3} \cdot \mathrm{Tag}$ & & & & $\begin{array}{l}0,012 \\
0,012\end{array}$ & $\begin{array}{c}0,011 \\
-\end{array}$ & $0, \overline{0} 11$ & 0,0296 & 0,0277 & 0,028 & & \\
\hline Reinigungseffekt. . . . .\% & & & & $\begin{array}{l}43 \\
46\end{array}$ & $\begin{array}{l}41 \\
-\end{array}$ & $\overline{44}$ & 46 & 43 & 43 & & \\
\hline
\end{tabular}


Tabelle 13

Versucbe mit Belebtscblammverfabren, Scblammeigenschaften, Scblammproduktion und Scblammzusammensetzung (Abscbnittsmittel)

(ÜS = Uberschußschlamm; Bel-B = Belüftungsbecken; RS = Rücklaufschlamm; RW = Rohwasser; TS = Trockenschlamm)

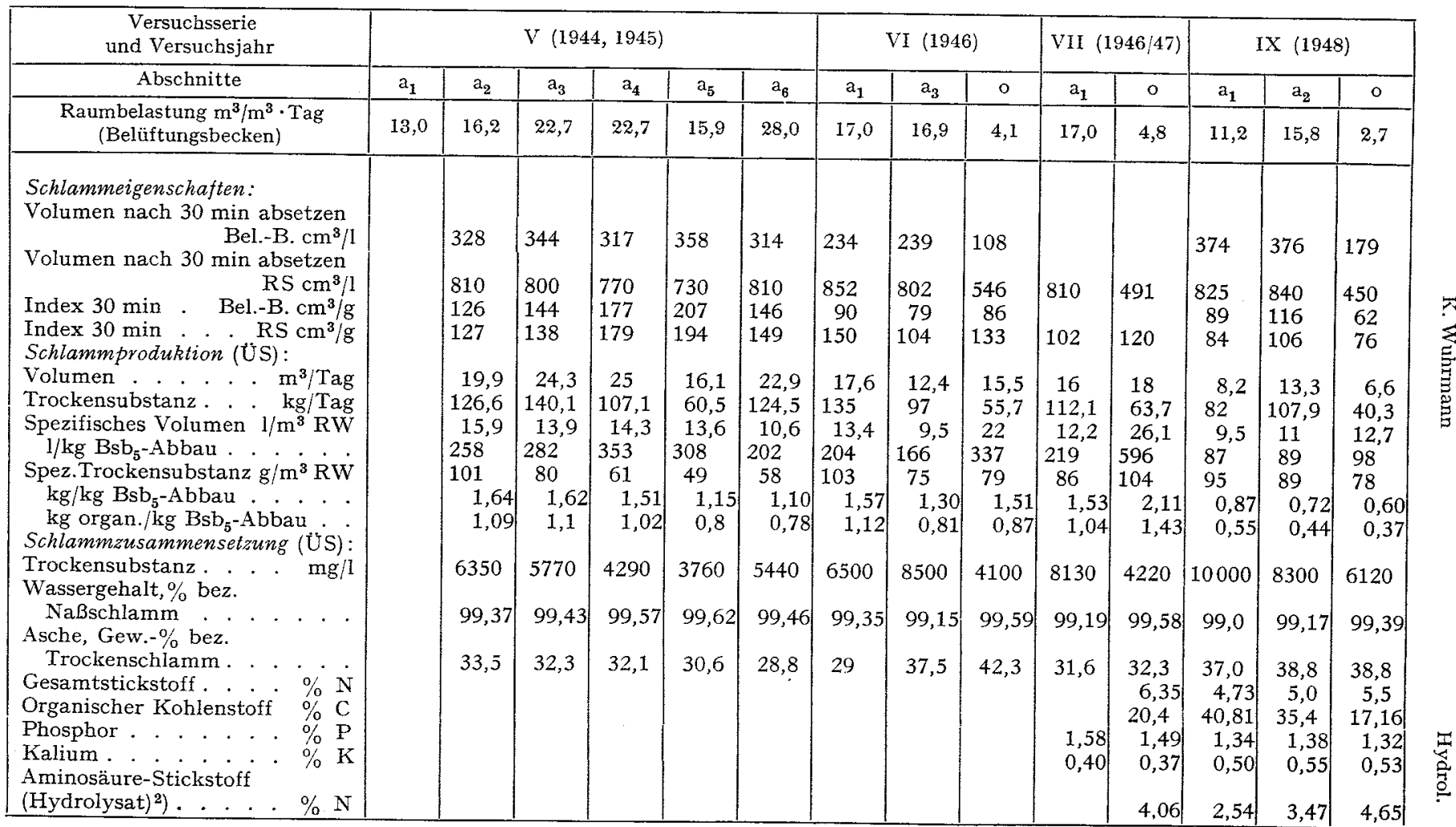


Tabelle 13 (Fortsetzung)

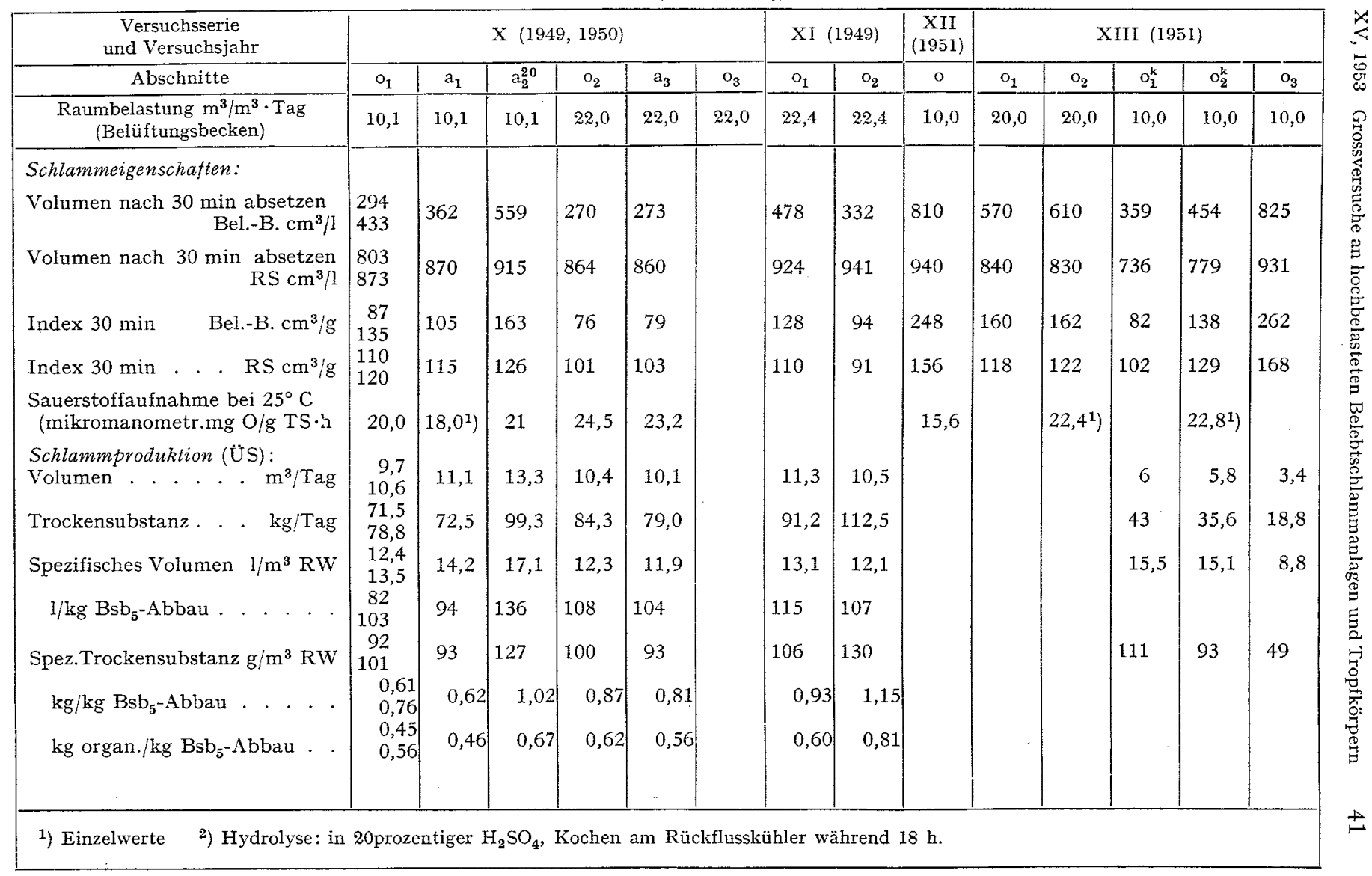


Tabelle 13 (Fortsetzung)

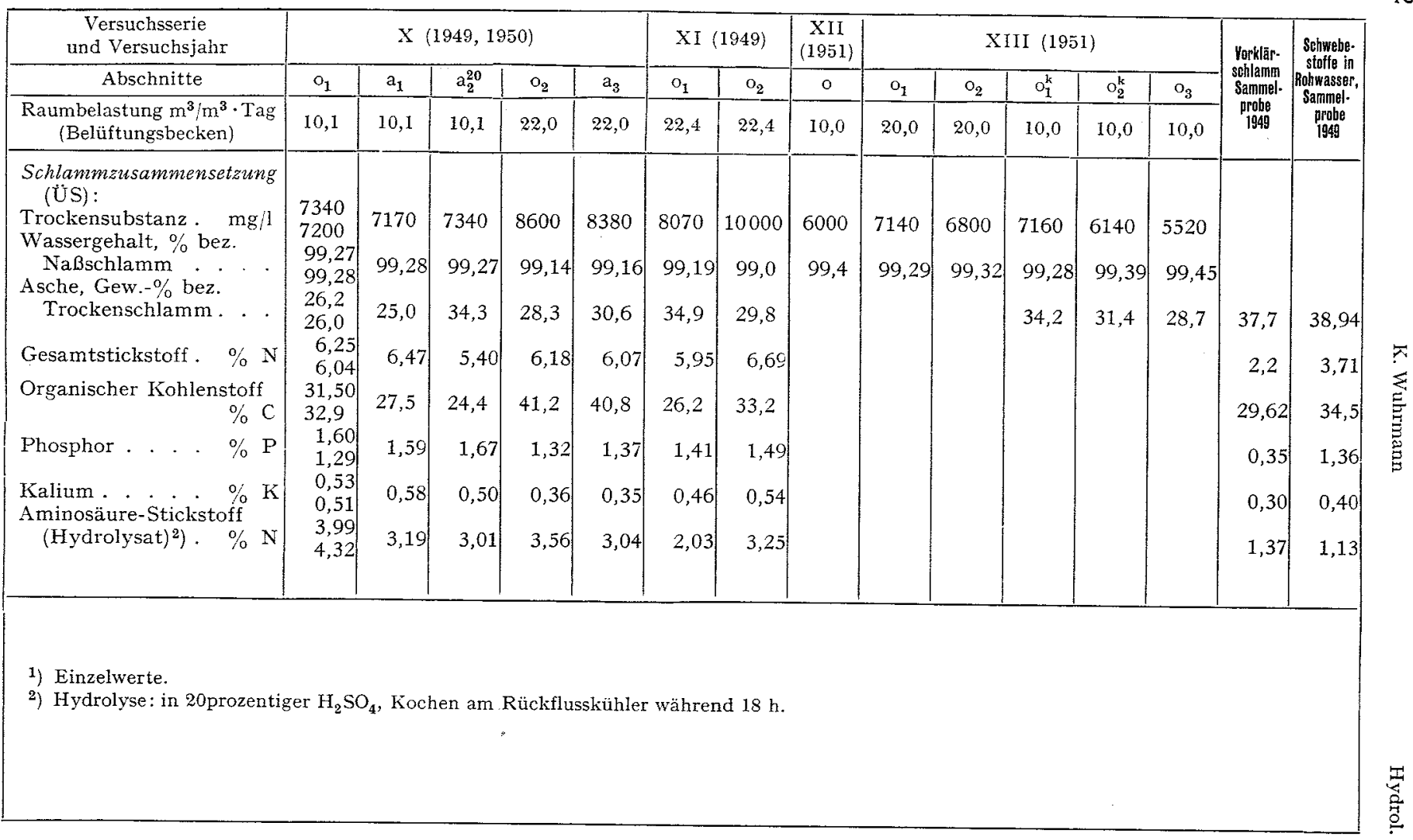


Tabelle 14

Versucbe mit künstlich belüftetem Tropfkörper, Versucbseinricbtungen und effektive Betriebsdaten

\begin{tabular}{|c|c|c|c|c|c|c|c|c|c|c|c|c|c|}
\hline $\begin{array}{l}\text { Versuchsserie } \\
\text { und Versuchsjahr }\end{array}$ & \multicolumn{6}{|c|}{$V(1944,1945)$} & \multicolumn{4}{|c|}{ VIII $(1947,1948)$} & \multicolumn{2}{|c|}{ IX (1948) } & \multirow[t]{2}{*}{$\begin{array}{c}\mathrm{X} \\
(1950) \\
\end{array}$} \\
\hline Abschnitte & 1 & 2 & 3 & 4 & 5 & 6 & 1 & 2 & 4 & 6 & I & 2 & \\
\hline $\begin{array}{c}\text { Raumbelastung } \mathrm{m}^{3} / \mathrm{m}^{3} \cdot \mathrm{Tag} \\
\text { (Belüftungsraum) }\end{array}$ & 4,9 & 6,2 & 8,7 & 10,0 & 7,3 & 12,9 & 7,0 & 7,0 & 9,7 & 9,7 & 5,0 & 10,0 & 12,0 \\
\hline $\begin{array}{l}\text { Oberflächenbelastung } \\
\text { (Belüftungsraum) m/Tag }\end{array}$ & 14,6 & 21,8 & 30,4 & 35,0 & 25,6 & 45,1 & 24,5 & 24,5 & 34,0 & 34,0 & 17,5 & 35,0 & 42,0 \\
\hline $\begin{array}{l}\text { Vorklärung: } \\
\text { Trockenwetter-Tagesmittel }\end{array}$ & & & & & & & & & & & & & \\
\hline Tropfkörper: $\quad \min$ & $\sim 40$ & $\sim 40$ & $\sim 40$ & $\sim 50$ & $\sim 50$ & $\sim 50$ & $\sim 40$ & $\sim 40$ & $\sim 40$ & $\sim 40$ & $\sim 40$ & $\sim 40$ & $\sim 85$ \\
\hline Volumen ...... $\mathrm{m}^{3}$ & 68 & 68 & 80 & 80 & 80 & 80 & 80 & 80 & 80 & 80 & 80 & 80 & 80 \\
\hline $\begin{array}{l}\text { Oberfläche } \\
\text { Nachklärbecken: }\end{array}$ & 23 & 23 & 23 & 23 & 23 & 23 & 23 & 23 & 23 & 23 & 23 & 23 & 23 \\
\hline 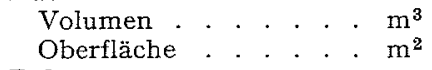 & $\begin{array}{l}47 \\
20\end{array}$ & $\begin{array}{l}47 \\
20\end{array}$ & $\begin{array}{l}47 \\
20\end{array}$ & $\begin{array}{l}47 \\
20\end{array}$ & $\begin{array}{l}47 \\
20\end{array}$ & $\begin{array}{l}47 \\
20\end{array}$ & $\begin{array}{l}47 \\
20\end{array}$ & $\begin{array}{l}47 \\
20\end{array}$ & $\begin{array}{l}47 \\
20\end{array}$ & $\begin{array}{l}47 \\
20\end{array}$ & $\begin{array}{l}47 \\
20\end{array}$ & $\begin{array}{l}47 \\
20\end{array}$ & $\begin{array}{l}47 \\
20\end{array}$ \\
\hline $\begin{array}{l}\text { Rohwassermenge: } \\
\text { Tag . . . . . . . . l/s }\end{array}$ & 3,9 & 5,8 & 8,1 & 9,32 & $\begin{array}{l}\max \\
=10\end{array}$ & $\begin{array}{c}6-24 h \\
15\end{array}$ & 6,5 & 6,5 & $\mid \begin{array}{c}6-18 h \\
15\end{array}$ & $\begin{array}{c}6-18 \mathrm{~h} \\
15\end{array}$ & $\begin{array}{c}7-18 h \\
6,3\end{array}$ & $\left|\begin{array}{c}7-18 \mathrm{~h} \\
12,7\end{array}\right|$ & $8-1630 \mathrm{~h}$ \\
\hline Nacht . . . . . . . . . . . l/s & 3,9 & 5,8 & 8,1 & 9,32 & $\min$ & $\begin{array}{c}24-6 h \\
3\end{array}$ & & & $\left|\begin{array}{c}18-6 h \\
3\end{array}\right|$ & $\begin{array}{c}18-6 h \\
3\end{array}$ & $\begin{array}{c}18-7 \mathrm{~h} \\
3,2\end{array}$ & $\begin{array}{c}18-7 h \\
6,3\end{array}$ & $\begin{array}{c}16.30-81 \\
8,6\end{array}$ \\
\hline $\begin{array}{l}\text { Variation Tagesmenge: Nacht- } \\
\text { menge } . \cdot \cdot \cdot \cdot \cdot \cdot \cdot \cdot \cdot\end{array}$ & $1: 1$ & $1: 1$ & $1: 1$ & $1: 1$ & $\begin{array}{c}\text { variabel } \\
\max .4: 1\end{array}$ & $5: 1$ & $1: 1$ & $1: 1$ & $5: 1$ & $5: 1$ & $2: 1$ & $2: 1$ & $1,83: 1$ \\
\hline $\begin{array}{l}\text { Rohwassermenge } \cdot \mathrm{m}^{3} / \mathrm{Tag} \\
\text { Künstliche Belüftung, } 24-\mathrm{h}-\end{array}$ & 336 & 500 & 700 & 805 & 590 & 1038 & 562 & 562 & 778 & 778 & 400 & 800 & 960 \\
\hline 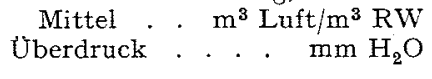 & 85 & 40 & 22 & 32 & $\begin{array}{r}38 \\
2\end{array}$ & $\begin{array}{r}29 \\
5\end{array}$ & 30 & $\begin{array}{l}50 \\
10\end{array}$ & $\begin{array}{l}50 \\
15\end{array}$ & $\left|\begin{array}{c}50 \\
45-135\end{array}\right|$ & $\begin{array}{r}46 \\
6\end{array}$ & $\begin{array}{l}46 \\
10\end{array}$ & $\begin{array}{c}\left.?^{2}\right) \\
>140\end{array}$ \\
\hline $\begin{array}{l}\text { Nachklärbecken: } \\
\text { Durchflussmenge: } \\
\text { Tag . . . . . . 1/s }\end{array}$ & 3,9 & 5,8 & $\left.6,5^{1}\right)$ & 6,5 & $\left.\max _{6,5}{ }^{1}\right)$ & 6,5 & 6,5 & 6,5 & $\left.6,5^{1}\right)$ & $\left.6,5^{1}\right)$ & 6,3 & $\left.6,3^{1}\right)$ & $\left.6,5^{1}\right)$ \\
\hline $\begin{array}{l}\text { Nacht } \cdot \text {. . . . . } \\
\text { Oberflächenbelastung: }\end{array}$ & 3,9 & 5,8 & 6,5 & 6,5 & $\min _{2,5}$ & 3,0 & 6,5 & 6,5 & 1,3 & 1,3 & 3,2 & 3,2 & 3,5 \\
\hline Tag . . . . . m/Tag & 16,8 & 25 & 28 & 28 & 28 & 28 & 28 & 28 & 28 & 28 & 27 & 27 & 28 \\
\hline Nacht $\cdot \cdot \cdot \mathrm{m} / \mathrm{Tag}$ & 16,8 & 25 & 28 & 28 & 10,8 & 13 & 28 & 28 & 5,6 & 5,6 & 13,8 & 13,8 & 15 \\
\hline $\begin{array}{l}\text { Theoretische Aufenthaltszeit: } \\
\text { Tag . . . . . . min }\end{array}$ & 200 & 135 & 120 & 120 & $\min$. & 120 & 120 & 120 & 120 & 120 & 124 & $\left.124^{1}\right)$ & $\left.120^{1}\right)$ \\
\hline Nacht . . . . . $\min$ & 200 & 135 & 120 & 120 & $\max$. & 260 & 120 & 120 & 600 & 600 & 248 & 248 & 220 \\
\hline
\end{tabular}

1) Nur ein Teil des TK-Abflusses durch Nachklärbecken. $\left.{ }^{2}\right)$ Ventilator auf maximaler Leistung $(\sim 450 \mathrm{l} / \mathrm{s})$. 
Tabelle 15

$V$ ersucbe mit künstlich belïftetem Tropfkörper, Analysenergebnisse (Abscbnittsmittelmerte)

$(\mathrm{RW}=$ Rohwasser; $\mathrm{A}=$ Abfluss-Nachklärbecken $)$

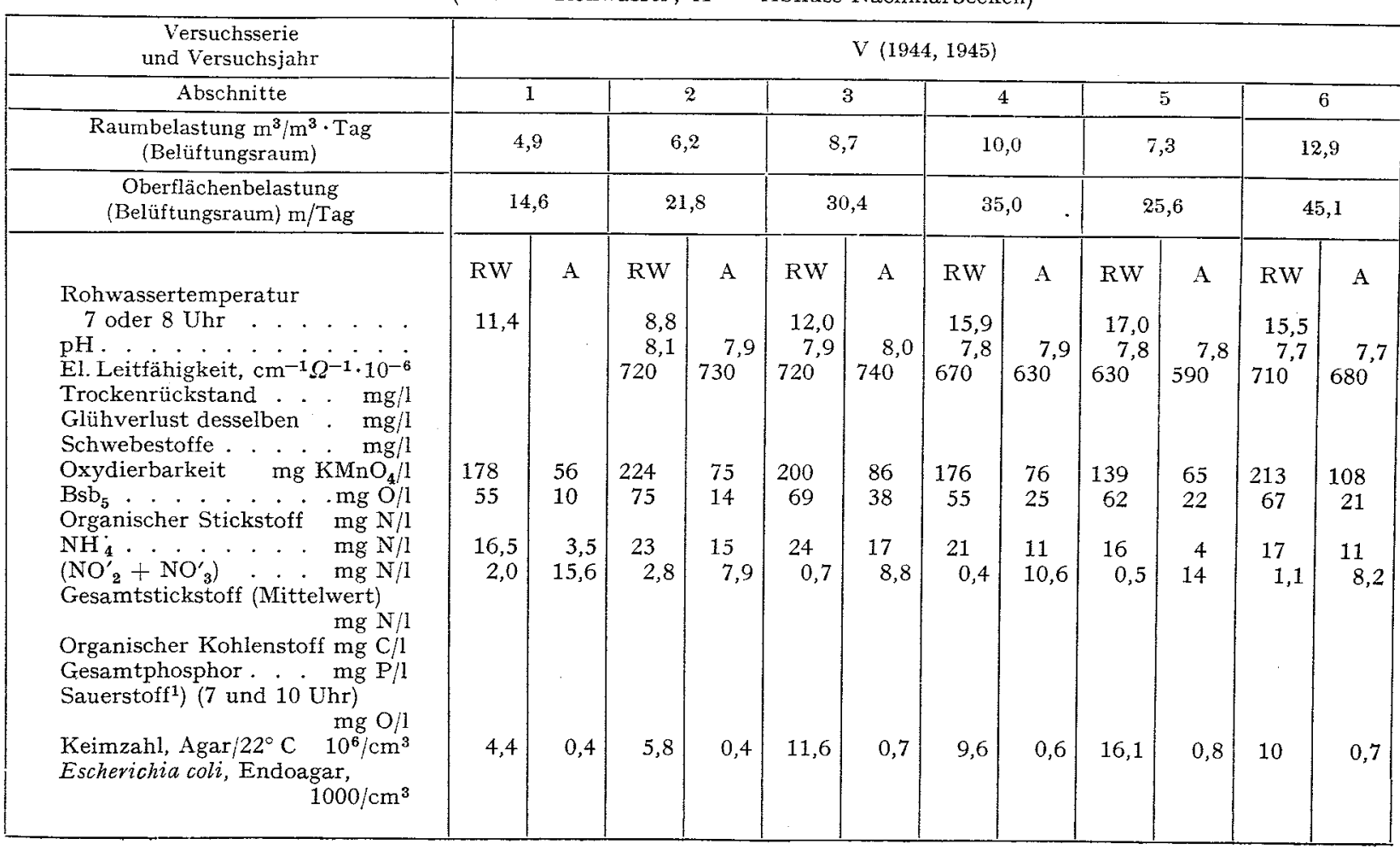


Tabelle 15 (Fortsetzung)

\begin{tabular}{|c|c|c|c|c|c|c|c|c|c|c|c|c|c|c|}
\hline $\begin{array}{l}\text { Versuchsserie } \\
\text { und Versuchsjahr }\end{array}$ & \multicolumn{8}{|c|}{ VIII $(1947,1948)$} & \multicolumn{4}{|c|}{ IX (1948) } & \multicolumn{2}{|c|}{$X(1950)$} \\
\hline Abschnitte & \multicolumn{2}{|c|}{1} & \multicolumn{2}{|c|}{2} & \multicolumn{2}{|c|}{4} & \multicolumn{2}{|c|}{6} & \multicolumn{2}{|c|}{ ] } & \multicolumn{2}{|c|}{2} & & \\
\hline $\begin{array}{c}\text { Raumbelastung } \mathrm{m}^{3} / \mathrm{m}^{3} \cdot \mathrm{Tag} \\
\text { (Belüftungsraum) }\end{array}$ & \multicolumn{2}{|c|}{7,0} & \multicolumn{2}{|c|}{7,0} & \multicolumn{2}{|c|}{9,7} & \multicolumn{2}{|c|}{9,7} & \multicolumn{2}{|c|}{5,0} & \multicolumn{2}{|c|}{10,0} & \multicolumn{2}{|c|}{12,0} \\
\hline $\begin{array}{l}\text { Oberflächenbelastung } \\
\text { (Belüftungsraum) } \mathrm{m} / \mathrm{Tag}\end{array}$ & \multicolumn{2}{|c|}{24,5} & \multicolumn{2}{|c|}{24,5} & \multicolumn{2}{|c|}{34,0} & \multicolumn{2}{|c|}{34,0} & \multicolumn{2}{|c|}{17,5} & \multicolumn{2}{|c|}{35,0} & \multicolumn{2}{|c|}{42,0} \\
\hline 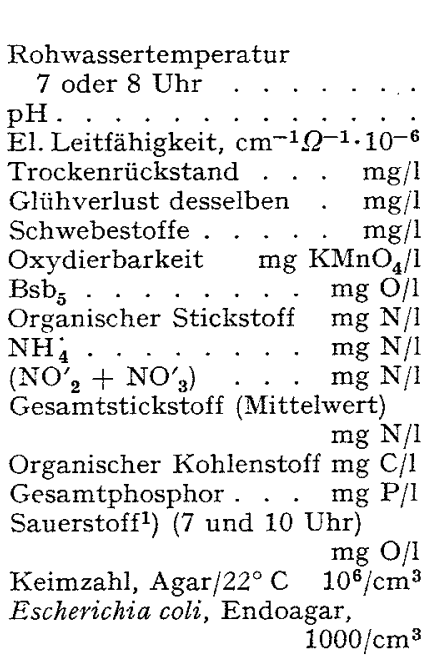 & $\begin{array}{r}\text { RW } \\
16,0 \\
7,6 \\
\\
\\
405 \\
145 \\
16,7 \\
14,2 \\
1,0 \\
31,9\end{array}$ & $\begin{array}{c}175 \\
49 \\
8,2 \\
4,2 \\
9,4 \\
21,8\end{array}$ & $\begin{array}{c}\mathrm{RW} \\
14,2 \\
7,9 \\
\\
\\
\\
380 \\
153 \\
15,7 \\
16,4 \\
0,8 \\
32,9\end{array}$ & $\begin{array}{c}145 \\
48 \\
5,2 \\
7,5 \\
9,2 \\
21,9\end{array}$ & $\begin{array}{c}\text { RW } \\
11,2 \\
7,9 \\
\\
\\
\\
520 \\
220 \\
16,0 \\
16,7 \\
1,5 \\
34,2\end{array}$ & $\begin{array}{r} \\
185 \\
65 \\
5,8 \\
14,0 \\
3,7 \\
23,5\end{array}$ & $\begin{array}{r}10,3 \\
13,1 \\
2,2 \\
\\
25,6\end{array}$ & $\begin{array}{r}4,7 \\
9,7 \\
3,4 \\
17,8\end{array}$ & $\begin{array}{c}\text { RW } \\
15,4 \\
\\
543 \\
222 \\
61 \\
123 \\
10,9 \\
12,3 \\
0,4 \\
\\
23,6\end{array}$ & $\begin{array}{r}437 \\
195 \\
13 \\
\\
20 \\
2,1 \\
4,7 \\
5,9 \\
12,7\end{array}$ & $\begin{array}{c}150 \\
7,22 \\
16,1 \\
0,4 \\
23,7 \\
3,53\end{array}$ & $\begin{array}{c}47 \\
3,28 \\
10,9 \\
3,7 \\
17,9 \\
2,63 \\
6,9\end{array}$ & \begin{tabular}{|c|} 
RW \\
14,1 \\
7,65 \\
568 \\
535 \\
220 \\
139 \\
322 \\
130 \\
8,7 \\
15,4 \\
0,63 \\
24,7 \\
81,9 \\
2,82 \\
\\
12,4 \\
47
\end{tabular} & $\begin{array}{c}\text { A } \\
\\
7,7 \\
552 \\
447 \\
160 \\
47 \\
165 \\
59 \\
4,8 \\
15,0 \\
0,6 \\
\\
20,4 \\
40,3 \\
2,32 \\
4,7 \\
8,8 \\
39\end{array}$ \\
\hline
\end{tabular}

1) Alle Sauerstoffgehalte gemessen im Abfluss des Tropfkörpers (vor Nachklärung). 
Tabelle 16

Versucbe mit künstlich belüftetem Tropfkörper, Reinigungs- bzw. Eliminationseffekte und Leistungen

(Alle Werte gerundet)

\begin{tabular}{|c|c|c|c|c|c|c|c|c|c|c|c|c|c|}
\hline $\begin{array}{l}\text { Versuchsserie } \\
\text { und Versuchsjahr }\end{array}$ & & & V $(194$ & , 1945) & & & & III (19 & 47,1948 & & IX & 1948) & $\begin{array}{c}\mathrm{X} \\
(1950)\end{array}$ \\
\hline Abschnitte & 1 & 2 & 3 & 4 & 5 & 6 & 1 & 2 & 4 & 6 & 1 & 2 & \\
\hline $\begin{array}{c}\text { Raumbelastung } \mathrm{m}^{3} / \mathrm{m}^{3} \cdot \text { Tag } \\
\text { (Belüftungsraum) }\end{array}$ & 4,9 & 6,2 & 8,7 & 10,0 & 7,3 & 12,9 & 7,0 & 7,0 & 9,7 & 9,7 & 5,0 & 10,0 & 12,0 \\
\hline $\begin{array}{l}\text { Oberflächenbelastung } \\
\text { (Belüftungsraum) } \mathrm{m} / \mathrm{Tag}\end{array}$ & 14,6 & 21,8 & 30,4 & 35,0 & 25,6 & 45,1 & 24,5 & 24,5 & 34,0 & 34,0 & 17,5 & 35,0 & 42,0 \\
\hline 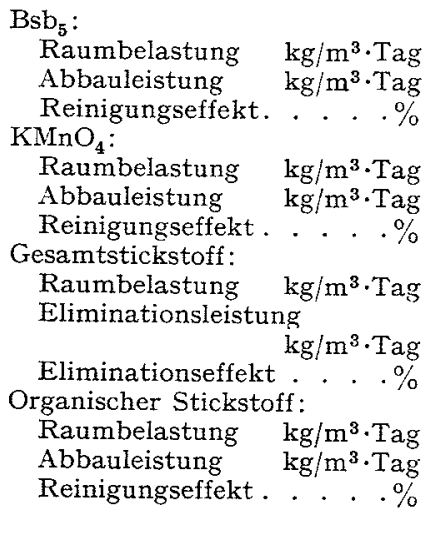 & $\begin{array}{l}0,27 \\
0,21 \\
80 \\
\\
0,87 \\
0,58 \\
67\end{array}$ & $\begin{array}{c}0,46 \\
0,37 \\
80 \\
\\
1,39 \\
0,89 \\
64\end{array}$ & $\begin{array}{l}0,60 \\
0,26 \\
44 \\
\\
1,74 \\
0,96 \\
55\end{array}$ & $\begin{array}{c}0,55 \\
0,30 \\
54 \\
\\
1,76 \\
0,99 \\
56\end{array}$ & $\begin{array}{l}0,45 \\
0,31 \\
68 \\
\\
1,01 \\
0,54 \\
53\end{array}$ & $\begin{array}{l}0,86 \\
0,60 \\
69 \\
\\
2,75 \\
1,38 \\
50\end{array}$ & \begin{tabular}{|l}
1,01 \\
0,67 \\
66 \\
2,84 \\
1,59 \\
56 \\
\\
0,22 \\
\\
0,067 \\
30,4 \\
0,117 \\
0,059 \\
50,6
\end{tabular} & $\begin{array}{c}1,07 \\
0,70 \\
65 \\
2,66 \\
1,62 \\
61 \\
0,23 \\
0,077 \\
33,4 \\
0,11 \\
0,073 \\
66,4\end{array}$ & $\begin{array}{c}2,13 \\
1,41 \\
66 \\
5,05 \\
3,18 \\
63 \\
0,33 \\
0,105 \\
31,3 \\
0,15 \\
0,097 \\
62,6\end{array}$ & $\begin{array}{c}0,25 \\
0,071 \\
28,6 \\
0,10 \\
0,052 \\
52,2\end{array}$ & $\begin{array}{c}0,118 \\
0,054 \\
46 \\
\\
0,054 \\
0,044 \\
81\end{array}$ & $\begin{array}{l}0,237 \\
0,058 \\
25 \\
0,072 \\
0,039 \\
55\end{array}$ & $\begin{array}{l}1,56 \\
0,85 \\
55 \\
3,87 \\
1,88 \\
49 \\
0,297 \\
0,052 \\
17 \\
0,104 \\
0,047 \\
45\end{array}$ \\
\hline
\end{tabular}


Tabelle 17

Versucbe mit künstlicb belüftetem Tropfkörper, Schlammproduktion und Schlammzusammensetzung

(Eingeklammerte Werte $=$ starke Schlammretention im Körper; RW = Rohwasser; $\mathrm{TS}=$ Trockenschlamm; alle Werte gerundet)

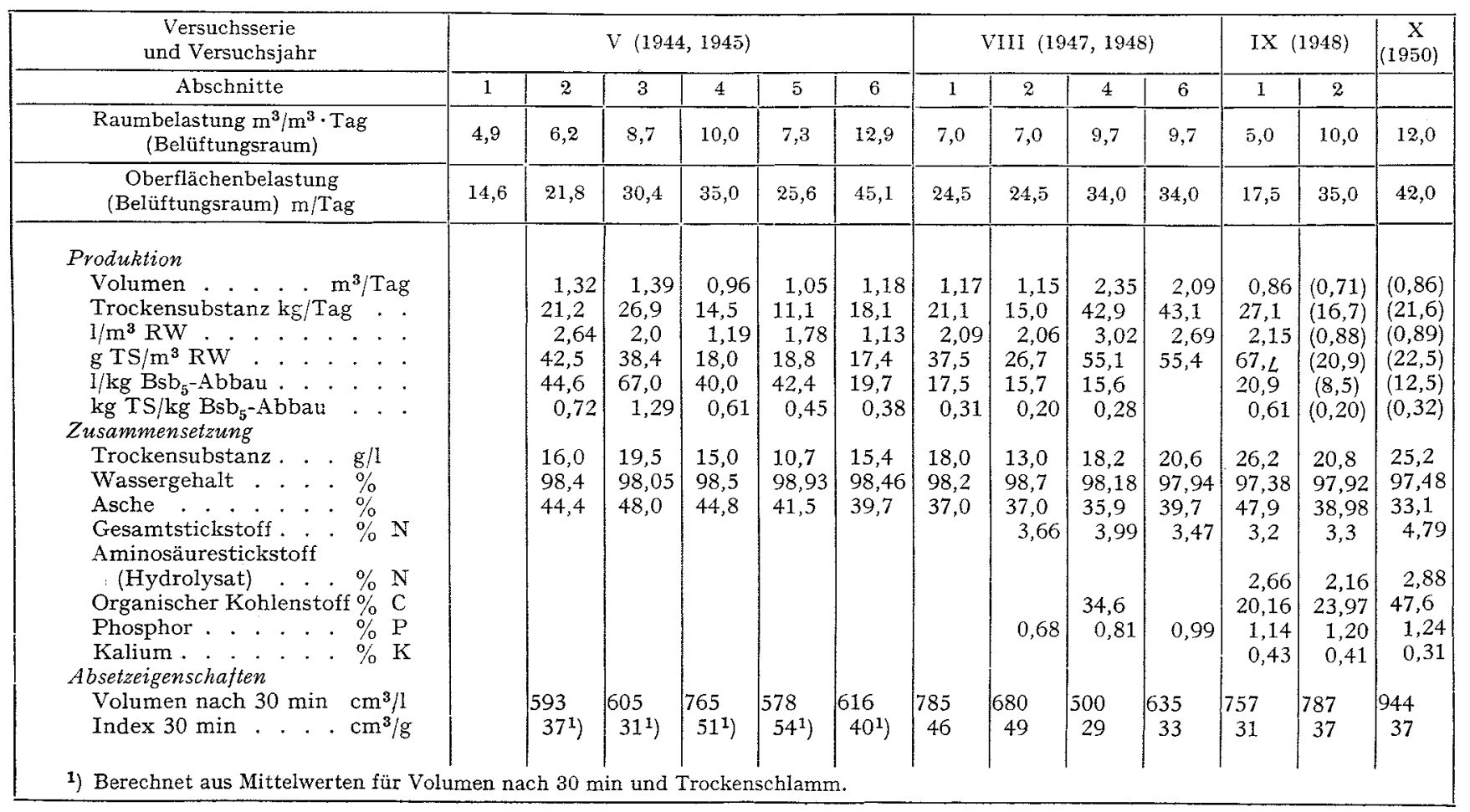


Bezüglich der Abwasserkonzentration können alle Versuchsserien seit I947 (Serie VIII) als massgebend für zürcherische Verhältnisse betrachtet werden, denn wir beobachteten seit diesem Zeitpunkte keine irgendwie ins Gewicht fallenden Veränderungen in Konzentration oder Zusammensetzung des Rohwassers mehr.

\section{Bemerkungen zu den Tabellen 9 bis 17}

Wir haben sämtliche uns für die genaue Umschreibung der Versuchsanordnungen und Versuchsergebnisse notwendig erscheinenden Zahlenangaben in den Tabellen 9 bis 17 zusammengefasst. Die Messungen wurden so gruppiert, dass Daten über Versuchseinrichtungen, Anlagenbetrieb, Analysenwerte usw. für alle Experimente auf der gleichen Tabelle gefunden werden können.

Die Angaben in den Tabellen stellen im allgemeinen Mittelmerte der in den einzelnen Versuchsabschnitten gewonnenen Einzelbeobachtungen dar. In der Regel wurden abgeleitete Zahlen, wie Reinigungseffekte, spezifische Schlammproduktionsmengen usw., aus den entsprechenden Abschnittsmittelwerten und nicht aus den Einzelbeobachtungen berechnet. In Anbetracht der in jedem Abschnittsmittelwert enthaltenen zahlreichen Einzelbeobachtungen ist dieses Verfahren statistisch erlaubt.

Aus Platzgründen wurde darauf verzichtet, die Streuungen der einzelnen Messwerte anzugeben. Soweit dies für die weitere Diskússion von Bedeutung ist, wird in den späteren Abschnitten darauf eingetreten. Wir möchten an dieser Stelle nochmals darauf aufmerksam machen, dass zum Beispiel Vergleiche zwischen einzelnen Versuchen erst nach sorgfältiger statistischer Beurteilung der Messungen vorgenommen werden können. Die zeitlichen Streuungen in der Zusammensetzung des Abwassers oder auch in einzelnen Betriebsdaten von Reinigungsanlagen sind so gross, dass auch Mittelwerten aus sehr zahlreichen Messungen eine beträchtliche Variabilität anhaftet, die bei einer vergleichenden Beurteilung unter allen Umständen zu berücksichtigen ist. 


\section{DRITTER TEIL}

\section{AUSWERTUNG DER BEOBACHTUNGEN}

Nachfolgend möchten wir die Ergebnisse der Versuche mit hochbelasteten Belebtschlammanlagen und künstlich belüfteten Tropfkörpern in der Weise zusammenfassend betrachten, dass bezüglich einzelner, uns wichtig erscheinender Fragen möglichst nach stetigen Beziehungen zwischen den Belastungen der Anlagen und den Ergebnissen hinsichtlich Abwasserreinigung, Schlammproduktion usw. gesucht wird. Wir belegen die Ausführungen mit entsprechenden Auszügen aus den früher genannten Tabellen bzw. statistischen Auswertungen der Einzelmessungen aus sämtlichen Versuchen. Soweit dies von Interesse ist, ziehen wir zum Vergleiche Erfahrungen von ausländischen Abwasserreinigungsanlagen heran, von denen uns genügend zuverlässige Analysenresultate zur Verfügung stehen.

\section{A. Bemerkungen zum Betriebe der Versuchsanlagen und den Verfahren im allgemeinen}

Wir haben bereits festgestellt, dass die Dimensionen der Versuchsanlagen unseres Erachtens gross genug sind, um die Beobachtungen bezüglich Leistungsfähigkeit verallgemeinern zu können. Dasselbe gilt auch für die Erfahrungen rein betriebsmässiger Art, wenn auch in dieser Hinsicht eine gewisse Einschränkung zu machen ist, weil das von uns verarbeitete Abwasser von sehr gleichmässiger und für die Reinigung von relativ günstiger Zusammensetzung war. Betriebsstörungen, die zum Beispiel ihre Ursache in Konzentrationsstössen spezieller industrieller Abwässer haben könnten, waren bei unsern Experimenten nicht zu befürchten. Gelegentliche, mehr oder weniger grosse Übertritte von Mineralöl aus der städtischen Kläranlage waren das einzige Störungsmoment für die Versuche. Irgendwelche bedeutsameren Folgen solcher «Verölungen» traten jedoch im Laufe der vielen Jahre weder bei den Belebtschlamm- noch bei den Tropfkörperversuchen auf.

\section{BEOBACHTUNGEN BEIM BELEBTSCHLAMMVERFAHREN}

\section{a) Belüftung und Scblammkonzentration im Belüftungsbecken}

Es ist leicht einzusehen, dass eine Belebtschlammanlage dann die grösste Reinigungsleistung zeigen muss, wenn sie mit der höchsten Schlammkonzentration im Belüftungsbecken betrieben wird, die mit der vor- 
handenen Belüftungseinrichtung in genügender Weise mit Sauerstoff versorgt werden kann. In Experimenten, über die in einer anderen Arbeit berichtet werden wird, konnten wir zeigen, dass die normale Atmungsintensität (Sauerstoffaufnahme) des Belebtschlammes aufrechterhalten bleibt, auch wenn der Sauerstoffgehalt im Schlammgemisch weniger als I $\mathrm{mg} \mathrm{O} / \mathrm{l}$ beträgt. Auf diese betriebstechnisch wichtige Tatsache machte schon VIEHL

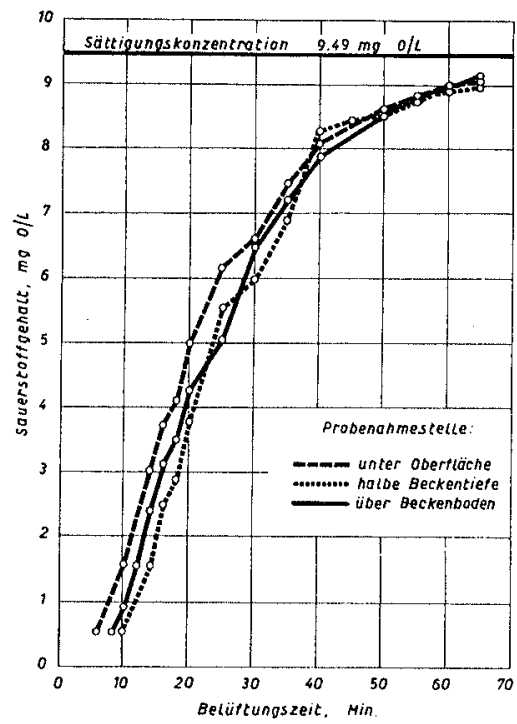

Abb. 10. Sauerstoffaufnahmekurven in einem Abteil einer Belüftungskammer, Inhalt = $38,5 \mathrm{~m}^{3}, 2,75 \mathrm{~m}$ Bürstenlänge, $178 \mathrm{U} . / \mathrm{min}$ und $9 \mathrm{~cm}$ Eintauchtiefe der Bürsten. Eisensulfatverfahren.

Ordinate: Sauerstoffgehalt des Wassers (mg O:1). Abszisse: Belüftungszeit (min).

(I940) aufmerksam. Sie gilt aber nur unter der Voraussetzung, dass mittels genügender Turbulenz dafür gesorgt ist, die Oberflächen der einzelnen Schlammflocken im Sauerstoffgleichgewicht mit der umgebenden Lösung zu halten. Begrenzender Faktor für die Schlammatmung in einer Belcbtschlammanlage ist somit nicht in erster I.inie die Sauerstoffspannung im Belüftungsbecken, sondern vor allem die Intensität der Durchmischung des Belebtschlammgemisches, solange die Sauerstoffeintragung durch die Belüftungseinrichtung und die Sauerstoffzehrung des Schlammes einander mindestens das Gleichgewicht halten.

KESSENER (I932) hat das Sumerstoffaufnuthmevernögen einer Anlage definiert als diejenige Sauerstoffmenge, welche pro Kubikmeter Beckeninhalt 
in einer Stunde bei $10^{\circ} \mathrm{C}$ Wassertemperatur und $760 \mathrm{~mm}$ Luftdruck in Lösung gebracht wird, wenn der Sauerstoffgehalt des Wassers o mg/l beträgt. Wir haben die Sauerstoffaufnahme in unseren Belüftungsbecken bei Bürstenbelüftung mit der von KESSENER angegebenen Eisensulfatmethode ebenfalls bestimmt. Nachstehend geben wir die Überlegungen bekannt, die sich auf Grund dieser Messungen anstellen lassen. Wir gehen beispielsweise von der Sauerstoffaufnahme unserer Belüftungsbecken aus, die sich bei der am häufigsten verwendeten Betriebsweise mit $9 \mathrm{~cm}$ Eintauchtiefe der Bürsten und einer Tourenzahl von $178 \mathrm{U} . / \mathrm{min}$ ergibt. Unter diesen Bedingungen erhielt man an drei Stellen in einer Belüftungskammer (unter Oberfläche, in halber Beckentiefe und am Beckenboden) die Sauerstoffaufnahmekurven nach Abbildung Io. Diese Kurven lassen sich bekanntlich durch die Gleichung der monomolekularen Umsetzung ausdrücken

worin:

$$
\frac{d c}{d \tau}=K \frac{c_{s}-c_{0}}{c_{s}-c_{\tau}},
$$

c Sauerstoffkonzentration im Wasser,

$\tau$ Zeit,

$c_{s}$ Sättigungskonzentration des Sauerstoffs im Wasser bei gegebener Temperatur und Luftdruck ( $\mathrm{mg} \mathrm{O} / \mathrm{l}$ ),

$c_{\circ}$ Sauerstoffkonzentration im Wasser zur Zeit $\tau_{\circ}(\mathrm{mg} \mathrm{O} / \mathrm{l})$,

$c_{\tau}$ Sauerstoffkonzentration im Wasser zur Zeit $\tau(\mathrm{mg} \mathrm{O} / \mathrm{l})$,

$K$ Sauerstoffaufnahmekoeffizient, das heisst die Geschwindigkeit der Sauerstoffaufnahme im Becken zu einer bestimmten Zeit $\tau$ ist dem in jenem Zeitpunkt herrschenden Sättigungsdefizit des Sauerstoffs proportional. $K$ wird nach Integration zwischen den Grenzen $\tau_{\mathrm{o}}$ und $\tau$ zu

$$
K=\ln \frac{c_{s}-c_{o}}{c_{s}-c_{r}} \cdot \frac{1}{\tau-\tau_{\circ}} .
$$

Aus den Kurven in Abbildung II lassen sich folgende Werte für $K$ ermitteln, wenn $\tau$ in Stunden gemessen wird:

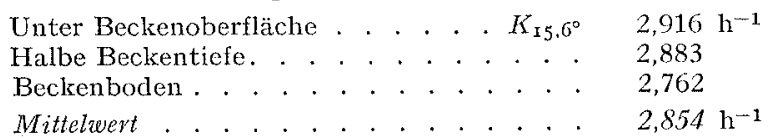

Die Sauerstoffaufnahme $W$ des Beliuftungsbeckens ergibt sich nun zu

$$
W=K c_{s} \mathrm{gO} / \mathrm{m}^{3} \cdot \mathrm{h} .
$$

Die Grösse von $W$ ist direkt proportional zu $c_{s}$ und damit stark von der Wassertemperatur und dem Luftdruck abhängig. Aber auch der Koeffi- 
zient $K$ bedarf einer Korrektur, wenn seine unter bestimmten Temperaturbedingungen ermittelten Werte für andere Wassertemperaturen verwendet werden sollen. PASvEER (I949, I950) hat in seinen eingehenden Untersuchungen über die Sauerstoffaufnahme von Wasser bei künstlicher Belüftung abgeleitet, dass $K$ als Produkt von zwei Faktoren aufgefasst werden kann, nämlich:

worin:

$$
K=2 \sqrt{\frac{k^{-}}{\pi}} \times \quad \frac{q}{v} \sqrt{\frac{1}{\tau^{\prime}}}
$$

$k$ Diffusionskonstante für Sauerstoff in Wasser $\left[\mathrm{cm}^{2} / \mathrm{h}\right]$, $q / p$ hypothetische Kontaktfläche Luft-Wasser pro Volumeneinheit Wasser $\mathrm{zu}$ einem bestimmten Zeitpunkt $\left[\mathrm{cm}^{-1}\right]$,

$\tau^{\prime}$ hypothetische Dauer des Bestandes der relativen Kontaktfläche $q / p[\mathrm{~h}]$, $K$ Sauerstoffaufnahmekoeffizient $\left[\mathrm{h}^{-1}\right]$.

Für eine gegebene Belüftungseinrichtung ist bei unveränderlichem Betrieb der Oberflächenfaktor $(q / \nu) \sqrt{1 / \tau^{\prime}}$ unabhängig von der Temperatur konstant. Dagegen ist die Diffusionskonstante $k$ temperaturabhängig. Unter Berücksichtigung der Viskosität des Wassers bei verschiedenen Temperaturen und auf Grund des von EINSTEIN und voN SMOLUCHOVSKI berechneten Wertes für

$$
k_{10^{\circ}}=\mathrm{I}, 06 \cdot \mathrm{IO}^{-5} \mathrm{~cm}^{2} / \mathrm{s}
$$

lässt sich $k$ für einige Temperaturen wie folgt berechnen:

Tabelle 18

Diffusionskonstanten pon Sauerstoff in WKasser bei perschiedenen Temperaturen

\begin{tabular}{|c|c|c|}
\hline $\begin{array}{c}\text { Temperatur } \\
{ }^{\circ} \mathrm{C}\end{array}$ & $\begin{array}{c}k_{t} \\
\mathrm{~cm}^{2} / \mathrm{s}\end{array}$ & $\begin{array}{c}k_{t} \\
\mathrm{~cm}^{2} / \mathrm{h}\end{array}$ \\
\hline 5 & $0,896 \cdot 10^{-5}$ & 0,03225 \\
10 & $1,06 \cdot 10^{-5}$ & 0,0382 \\
15 & $1,235 \cdot 10^{-5}$ & 0,0445 \\
20 & $1,428 \cdot 10^{-5}$ & 0,0514 \\
25 & $1,631 \cdot 10^{-5}$ & 0,0588 \\
\hline
\end{tabular}

An Hand des experimentell bestimmten Wertes für $K=2,854 \mathrm{~h}^{-\mathrm{x}}$ bei I $5,6^{\circ} \mathrm{C}$ in unserer Versuchsanlage und dem « Diffusionsfaktor» bei I $5,6^{\circ} \mathrm{C}$ von

$$
2 \sqrt{\frac{k_{15,6^{\circ}}}{\pi}}=0,242
$$


findet man aus Gleichung (4) den temperaturunabhängigen «Oberflächenfaktor»

$$
\frac{q}{v} \sqrt{\frac{1}{\tau^{\prime}}}=\frac{K_{15,6^{\circ}}}{2 \sqrt{\frac{k_{15,6^{\circ}}}{\pi}}}=\frac{2,854}{0,242}=\mathrm{II}, 79_{3} \mathrm{~cm}^{-\mathrm{I}} \mathrm{h}^{-\mathrm{I} / 2} .
$$

Mit Hilfe der für verschiedene Temperaturen berechneten Werte von $k_{t}$ (Tabelle I8) lassen sich nun die Sauerstoffaufnahmekoeffizienten $K_{t}$ bei denselben Temperaturen wie folgt berechnen:

$$
K_{t}=2 \sqrt{\frac{k_{t}}{\pi}} \cdot \mathrm{II}, 79_{3} \mathrm{~h}^{-1},
$$

wobei man nachfolgende Grössen erhält:

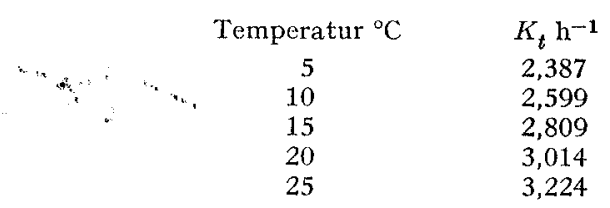

Man findet somit für das Sauerstoffaufnahmevermögen $W_{t}$ bei verschiedenen Temperaturen gemäss Gleichung (3) folgende Werte, wenn man die entsprechenden Sauerstoffsättigungen $c_{s}$ für den mittleren Luftdruck von $728 \mathrm{~mm}$ auf unserer Versuchsanlage zugrunde legt:

Tabelle 19

Sauerstoffaufnabmevermögen des Belüftungsbeckens bei $178 \mathrm{U} \cdot / \mathrm{min}$

mit $9 \mathrm{~cm}$ Eintauchtiefe der Bürsten

\begin{tabular}{|c|c|c|c|}
\hline $\begin{array}{c}\text { Wassertemperatur } \\
{ }^{\circ} \mathrm{C}\end{array}$ & $\begin{array}{c}\left.c_{s}{ }^{\mathbf{}}\right) \\
\mathrm{mg} \mathrm{O} / \mathrm{l}\end{array}$ & $\begin{array}{c}K_{t} \\
\mathrm{~h}^{-1}\end{array}$ & $\begin{array}{c}W_{t} \\
\mathrm{~g} \mathrm{O} / \mathrm{m}^{\mathbf{3}} \cdot \mathrm{h}\end{array}$ \\
\hline \multirow{5}{5}{} & 12,19 & 2,387 & 29,2 \\
10 & 10,77 & 2,599 & 28,0 \\
15 & 9,56 & 2,809 & 26,9 \\
20 & 8,66 & 3,014 & 26,1 \\
25 & 7,83 & 3,224 & 25,2 \\
\hline
\end{tabular}

1) Unter Berücksichtigung der Wasserdampfspannung bei $t^{\circ}$ und $728 \mathrm{~mm}$ Luftdruck.

Sofern nun auch die Sauerstoffaufnahme eines Belebtschlammgemisches im Belüftungsbecken bekannt ist, lässt sich ohne weiteres feststellen, welche maximalen Schlammgehalte in der Anlage gehalten werden dürfen, damit gerade Gleichgewicht zwischen Sauerstoffversorgung und Sauerstoffzehrung gewährleistet ist. Die Sauerstoffmenge, die mit dem zu- 
fliessenden Abwasser in das Belüftungsbecken eingebracht wird, kann bei dieser Berechnung vernachlässigt werden, da sie relativ gering ist (sie wirkt gewissermassen als «stille Reserve»).

Die Sauerstoffzehrung $Q$ des Belebtschlammes wurde in einer Reihe von Versuchsserien laufend mikromanometrisch bei $25^{\circ} \mathrm{C}$ bestimmt (vgl. Tabelle I3). Die Temperaturabhängigkeit der Schlammatmung wurde ebenfalls untersucht und soll in einer speziellen Arbeit später genauer dargestellt werden. Wir verwenden an dieser Stelle lediglich den gefundenen mittleren Temperaturkoeffizienten der Sauerstoffaufnahme des Schlammes, der auf Grund der Beobachtungen vorläufig wie folgt ausgedrückt werden soll:

$$
\alpha=\frac{\log Q_{t_{1}}-\log Q_{t_{2}}}{t_{1}-t_{2}} \not>c^{-1}
$$

worin $Q_{t_{1}}$ und $Q_{t_{2}}$ auf die Einheit bezogen sind, und ferner bedeuten:

$\alpha \quad$ Temperaturkoeffizient der Schlammatmung $\left[b-1,\left[c^{-1}\right]\right.$

$Q_{t_{1}} Q_{t_{2}}$ Schlammatmung bei den Temperaturen $t_{1}$ und $t_{2}$,

$\mathrm{mg} \mathrm{O} / \mathrm{g}$ Trockensubstanz und Stunde,

$t_{1}, t_{2}$ Temperatur, ${ }^{\circ} \mathrm{C}$.

Die vorstehende empirische Beziehung stimmt nicht mit der Gleichung von VAN T'HOFF und ARRHENIUS überein, wird aber den Beobachtungen besser gerecht. $\alpha$ kann für die Grundatmung von üblichem Belebtschlamm zU 0,03 I bis 0,03 I5 angenommen werden. SAWYER und Mitarbeiter (I939) haben eine etwas andere empirische Formulierung für die Temperaturabhängigkeit der Schlammatmung bei ihren Versuchen gefunden, doch ergibt ihr Temperaturkoeffizient der Atmung Werte analoger Grösse wie bei unseren Experimenten, obschon sie Schlämme aus Vollreinigungsanlagen mit bedeutend geringerer absoluter Sauerstoffaufnahme untersucht haben

Tabelle 20

Temperaturabhängigkeit des Sauerstoff perbrauches von Belebtschlämmen verscbiedener Grundatmung

\begin{tabular}{|c|c|c|c|}
\hline \multirow{2}{*}{ Temperatur ${ }^{\circ} \mathrm{C}$} & \multicolumn{3}{|c|}{ Sauerstoffzehrung des Schlammes mg O/g $\cdot \mathrm{h}$} \\
\cline { 2 - 4 } & 15 & 20 & 25 \\
\hline & 3,60 & 4,80 & 6,0 \\
5 & 5,15 & 6,85 & 8,57 \\
10 & 7,35 & 9,80 & 12,2 \\
15 & 10,6 & 14,15 & 17,7 \\
20 & 15,0 & 20,0 & 25,0 \\
25 & & & Grundatmung $\left(25^{\circ} \mathrm{C}\right)$ \\
\hline
\end{tabular}


als wir (8- $\mathrm{I} 2 \mathrm{mg} \mathrm{O} / \mathrm{g} \cdot \mathrm{h}$ bei $25^{\circ} \mathrm{C}$ ), und sie eine andere Methode der Messung der Schlammatmung verwendeten. Für die Grundatmung von Schlämmen, die zwischen $\mathrm{I} 5$ und $25 \mathrm{mg} \mathrm{O} / \mathrm{g} \cdot \mathrm{h}$ bei $25^{\circ} \mathrm{C}$ beträgt, ergeben sich bei verschiedenen Temperaturen die Werte der Tabelle 20.

Aus diesen Daten kann nun berechnet werden, welche maximale Schlammkonzentration bei verschiedenen Schlammeigenschaften und Abwassertemperaturen in unserer Anlage gestattet sind, damit Sauerstoffaufnahme und -zehrung im Gleichgewicht stehen. Voraussetzung ist die Belüftung bei einer Tourenzahl der Bürsten von $178 \mathrm{U} . / \mathrm{min}$ und mit der Eintauchtiefe von $9 \mathrm{~cm}$. Diese zulässige Scblammkonzentration ergibt sich nämlich einfach aus

$$
C_{m a x}=\frac{W_{t}}{Q_{t}}
$$

wenn $Q_{t}$ in $\mathrm{mg} \mathrm{O} / \mathrm{g} \cdot \mathrm{h}$ bei $t^{\circ}$ gemessen wird. In Abbildung II sind für vorstehend genannte Grössen der Schlammatmung (welche die bisher beobachteten Atmungsintensitäten einschliessen) die in unserer Anlage im Maximum zulässigen Schlammkonzentrationen in Funktion der Abwassertemperatur aufgetragen. In dem bei uns üblichen Temperaturbereich von $\sim 9$ bis $16,5^{\circ} \mathrm{C}$ bewegen sich diese Konzentrationen zwischen rund $2000 \mathrm{mg} / \mathrm{l}$ (höchste Abwassertemperatur und höchste Schlammatmung)

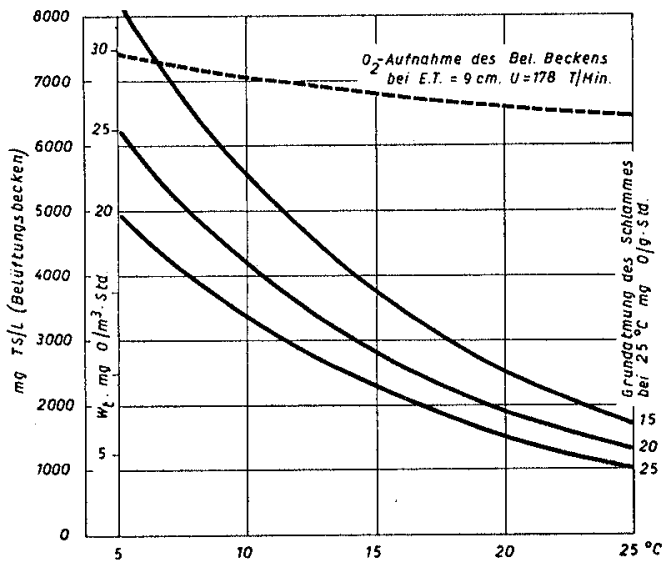

Abb. 11. Zusammenhang zwischen Sauerstoffaufnahmevermögen, Temperatur und zulässigem Schlammgehalt in unserer Versuchsanlage. Ordinate: Zulässige Schlammkonzentration im Belüftungsbecken (mg Trockensub$\operatorname{stanz} / 1)$ bzw. Sauerstoffaufnahmevermögen der Anlage $\left(\mathrm{g} \mathrm{O} / \mathrm{m}^{3} \cdot \mathrm{h}\right)$. Abszisse: Temperatur $\left({ }^{\circ} \mathrm{C}\right)$.

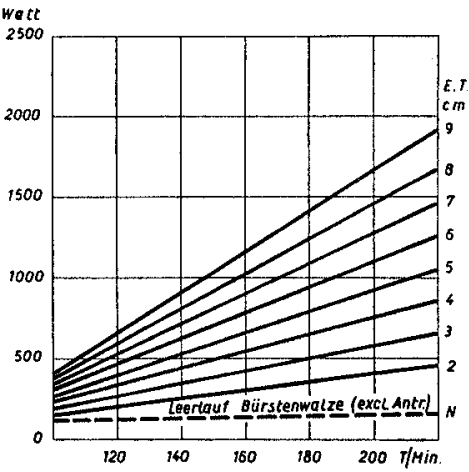

Abb. 12. Nettokraftverbrauch der Bürsten in Funktion von Eintauchtiefe und Tourenzahl. Ordinate: Kraftverbrauch (W). $\mathrm{Ab}$ szisse: Tourenzahl (U./min). Parameter: Eintauchtiefe (cm). 
und rund $5000-6000 \mathrm{mg} / \mathrm{l}$ (bei niedrigster Temperatur und Atmungs. intensität).

Prüft man die in den Tabellen Io, II und I3 enthaltenen Angaben über die in den einzelnen Versuchsabschnitten effektiv beobachteten Schlammkonzentrationen, Atmungsgrössen und Abwassertemperaturen, so stellt man sofort fest, dass gelegentlich beträchtliche Überschreitungen der mittleren Schlammkonzentration über das zulässige Maximum vorgekommen sind, ohne dass sich dabei allerdings besondere Betriebsschwierigkeiten gezeigt hätten. Man darf nämlich nicht vergessen, dass sowohl die Schlammkonzentration wie auch die Schlammatmung innerhalb eines Tages starken Schwankungen unterworfen sind (periodisches Ablassen des Überschussschlammes, Wechsel der Belastung des Schlammes mit Schmutzstoffen), und ferner eine zusätzliche Belüftung besonders während der Nacht durch den Sauerstoffgehalt des Abwassers (die vorstehend als «stille Reserve» bezeichnet wurde) eintritt. Wir haben denn auch regelmässig beobachtet, dass frühmorgens ein Sauerstoffüberschuss in den Belüftungsbecken vorhanden war, der im Laufe des Tages allmählich verschwand, um sich während der Nacht erneut wieder einzustellen.

\section{b) Wartung der Belebtschlammanlage}

Wir haben in Abschnitt I, C 2, bereits das Einfabren der Belebtschlammanlage geschildert. Der Dauerbetrieb gestaltete sich nachher in allen Versuchen äusserst einfach, indem sich - entgegen der allgemein verbreiteten Auffassung - nur eine geringe Wartung als notwendig ermies. Auf Grund unserer Erfahrungen möchten wir ausdrücklich feststellen, dass die häufig geäusserte Behauptung, wonach hochbelastete Belebtschlammanlagen schwierig zu bedienen seien und häufig zu Störungen Anlass geben sollen, keineswegs zutrifft. Dies gilt mindestens für so lange, als ein normales städtisches Abwasser zu reinigen ist. Es besteht kein Unterscbied zmischen dem Betrieb einer bocbbelasteten und einer scbmachbelasteten Anlage.

Da die Reinigungsleistung bei gegebener Raumbelastung $R_{h}{ }^{1}$ ) und $R_{b}^{2}$ ) in erster Linie vom optimalen Schlammgehalt im Belüftungsbecken abhängig ist, kommt diesem Faktor betriebsmässig grosse Bedeutung zu. Die Bestimmung des Sauerstoffaufnahmevermögens der Belïftungsbecken bietet nun auf Grund der Ausführungen im vorherigen Abschnitt die sicherste Grundlage für die Erstellung einer Betriebsvorschrift bezüglich

1) $R_{h}=$ hydraulische Raumbelastung, $\mathrm{m}^{3} / \mathrm{m}^{3} \cdot$ Tag.

2) $R_{b}=$ Belastung mit $\mathrm{Bsb}_{5}, \mathrm{~kg} / \mathrm{m}^{3} \cdot \mathrm{Tag}$. 
der einzuhaltenden Belebtschlammkonzentration. Würde noch die Schlammatmung und die Schlammkonzentration im Belüftungsbecken ständig verfolgt, so könnte damit theoretisch eine Anlage dauernd auf optimaler Leistung gehalten werden. Diese Messungen kommen aber zum mindesten auf Kleinanlagen nicht in Frage und sind auch gar nicht notwendig. An ihre Stelle tritt vorteilhafterweise eine Kontrolle des Sauerstoffgehaltes, zum Beispiel im Nachklärbecken. Lässt sich nämlich dort gerade noch Sauerstoff nachweisen $(\sim 0,2-0,5 \mathrm{mg} \mathrm{O} / \mathrm{l})$, so ist man sicher, dass das Belüftungsbecken eine genügende Sauerstoffversorgung aufweist. Die Regulierung des Schlammgehaltes ist dann mit der Dosierung des Rücklaufschlammes und des Überschußschlammes leicht möglich. Die Erfahrungen zeigen, dass die Einhaltung der zulässigen Schlammkonzentration nach einiger Zeit auch von ungeschultem Personal ohne weiteres beherrscht wird, wenn folgende Beobachtungen ein- bis dreimal wöchentlich angestellt werden:

a) Absetzversuch mit Belebtschlamm aus den Belüftungsbecken im Imhoff-Glas;

b) qualitative Beurteilung des Sauerstoffgehaltes im Nachklärbecken (Ansatz einer $\mathrm{O}_{2}$-Bestimmung nach WINKLER und qualitative Beurteilung der Bräunung des Niederschlages in der $\mathrm{O}_{2}$-Flasche);

c) subjektive Beurteilung der Flockung, Farbe und Absetzgeschwindigkeit des Schlammes im Imhoff-Glas.

In Grossanlagen, die sich naturgemäss träger verhalten als unser Kleinbetrieb, werden die qualitativen Beobachtungen vorteilhaft durch quantitative Messungen der Schlammkonzentration und des Sauerstoffgehaltes ergänzt.

Es ist eine Selbstverständlichkeit, dass zu den normalen Wartungsarbeiten die Reinbaltung der Anlagen gehört, die sich auf das regelmässige Abbürsten von Überlaufkanten, Abflussrinnen, offenen Schlammkanälen usw. erstrecken muss.

Bezüglich der Rücklaufschlammenge sind wir der Auffassung, dass es sich in kleineren Anlagen nicht lohnt, komplizierte Dosierungswechsel zwischen Tages- und Nachtbetrieb vorzunehmen. Es wird mit Vorteil eine konstante Fördermenge eingestellt, die sich nach den Ergebnissen der Beobachtungen betreffend Schlammgehalt richtet und im allgemeinen selten geändert werden muss. Das Rücklaufschlammverhältnis ist bei hochbelasteten Anlagen etwas höher anzusetzen (40-60\%) als bei schwachbelasteten (30 bis $50 \%$ ). In zahlreichen Versuchen hatten wir mit noch höheren Rück- 
laufmengen zu arbeiten, doch rührte dies ausschliesslich von den hydraulischen Voraussetzungen auf unserer Anlage her (Erzielung genügender Schlammgeschwindigkeiten in den Leitungen, um Verstopfungen zu vermeiden). Der Rücklaufschlamm bietet im übrigen die Möglichkeit, Perioden mit verhältnismässig schlechten Absetzeigenschaften des Schlammes zu überwinden, indem das Rücklaufverhältnis erhöht wird. Voraussetzung dazu ist allerdings das Vorhandensein einer Reservepumpe.

Die Menge des mindestens alle zwei Tage abzulassenden Überschussschlammes ergibt sich aus der Erfahrung und kann überdies grössenordnungsmässig auf Grund der Angaben in Abschnitt III, E I, abgeschätzt werden. Wir möchten darauf hinweisen, dass es wichtig ist, den Überschußschlamm langsam zu entfernen, um eine möglichst hohe Konzentration für das gesamte abgelassene Volumen zu erreichen. Dies gilt sowohl für den direkten Überschußschlammablass aus dem Nachklärbecken wie auch für den Fall einer Abzapfung aus den Rücklaufschlammleitungen. Bei Kleinanlagen hat sich der Ablass am Nachmittag, das heisst nach Verarbeitung der höchsten Schmutzstoffmenge in der Anlage, als günstig erwiesen. Bei grösseren Anlagen (über $3000 \mathrm{EW}$-Gleichwerte) ist ein kontinuierlicher Überschußschlammabfluss (zum Beispiel zu einem Eindickungsbecken) empfehlenswert.

\section{c) Bürstenbelïftung}

Die Bürstenbelüftung oder andere rein mechanische Belüftungseinrichtungen besitzen den grossen Vorteil, dass die einmal bestimmte Leistung bezüglich Sauerstoffeintragung in das Belebtschlammgemisch konstant bleibt und damit eine sichere Grundlage für die Betriebsweise einer Anlage bietet. Bei Druckluftbelüftung aus Filterplatten ist immer mit allmählicher Verstopfung der Diffusoren und damit einem abnehmenden Sauerstoffaufnahmevermögen der Belüftungskammern zu rechnen. Dieser Nachteil kann lediglich mittels der «grossblasigen» Belüftung aus offenen Rohren umgangen werden. Die Bürstenbelüftung hat sich auch wirtschaftlich als vorteilhaft erwiesen, denn auf unserer Anlage ist gemäss Tabelle ro und Abbildung I2 mit folgendem Kraftverbrauch zu rechnen:

$5,5 \mathrm{~m}$ Bürstenlänge pro $77 \mathrm{~m}^{3}$ Beckeninhalt

\begin{tabular}{|c|c|c|}
\hline $\begin{array}{l}\text { Kraftverbrauch der Bürsten bei } 178 \mathrm{U} . / \mathrm{min} \\
\text { und } 9 \mathrm{~cm} \text { Eintauchtiefe. }\end{array}$ & $=$ & $1,48 \mathrm{~kW}$ \\
\hline Sauerstoffaufnahmevermögen bei $10^{\circ} \mathrm{C}$ und & & \\
\hline 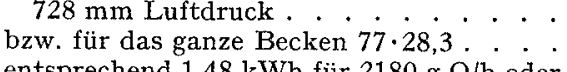 & $\begin{array}{l}= \\
=\end{array}$ & $\begin{aligned} 28,30 \mathrm{~g} \mathrm{O} / \mathrm{m}^{3} \cdot \mathrm{h} \\
2180,00 \mathrm{~g} \mathrm{O} / \mathrm{h}\end{aligned}$ \\
\hline entsp & $=$ & $\mathrm{V} / \mathrm{g} \mathrm{O}$ \\
\hline
\end{tabular}


Vergrössert man die Bürstenlänge pro Kubikmeter Beckeninhalt, so kann naturgemäss die Sauerstoffaufnahme des Systems wesentlich erhöht werden, so dass auch die Belastbarkeit der Becken mit Schmutzstoffen zunimmt. Dabei erniedrigt sich gleichzeitig der Kraftverbrauch pro Gramm aufgenommenen Sauerstoffs im gleichen Verhältnis. Wie dies PASVEER (I95I) gezeigt hat, ist es deshalb ausserordentlich lohnend, die Bürstenbelüftung noch weiter zu vervollkommnen. Da von den meisten in Betrieb stehenden Belebtschlammanlagen keine Angaben über das Sauerstoffaufnahmevermögen bekannt sind, kann ein Betriebskostenvergleich der Belïftungseinrichtung mit unseren Versuchen nur auf der Grundlage des Energieverbrauchs (für die Belüftung) pro Kilogramm $\mathrm{Bsb}_{5}$-Abbau erfolgen. Nimmt man gemäss IMHOFF-FArR überschlagsmässig für die Druckluftbelüftung $17,8 \mathrm{Wh} / \mathrm{m}^{3}$ Luft (entsprechend $1000 \mathrm{cuft} / \mathrm{min}=$ $4 \mathrm{hP}$ ) an, so lässt sich folgende Tabelle erstellen, die wir auf Angaben von IMHOFF (S. I62) stützen:

Tabelle 21

Energieverbrauch der Bürstenbelïftung und Druckluftbelüftung. pro Kilogramm Bs $b_{5}-$ Abbau

\begin{tabular}{|c|c|c|c|c|c|c|c|}
\hline \multicolumn{4}{|c|}{ Bürstenbelüftung } & \multicolumn{4}{|c|}{ Druckluftbelüftung } \\
\hline \multirow{2}{*}{ Versuch } & \multirow{2}{*}{$R_{b}$} & $\mathrm{kWh}$ & \multirow{2}{*}{$\eta \%$} & \multirow{2}{*}{ Anlage } & \multirow{2}{*}{$R_{b}$} & \multirow{2}{*}{$\frac{\mathrm{kWh}}{\mathrm{kg} \mathrm{Bsb_{5 }}}$} & \multirow{2}{*}{$\eta \%$} \\
\hline & & $\mathrm{kg} \mathrm{Bsb}$ & & & & & \\
\hline Serie $x_{1}$ & $1,4-1,7$ & $0,27-0,29$ & $91-93$ & New York, W. I. & 1,08 & 0,42 & 94 \\
\hline Serie $\mathrm{IX}_{2}$ & 2,36 & 0,21 & 83 & New York, Bow.B. & $0,8-1,3$ & 0,54 & 90 \\
\hline Serie $\mathrm{X}_{2}^{2}$ & 3,06 & 0,17 & $82-83$ & New York, Jamaica & 1,6 & 0,39 & 75 \\
\hline Serie $\mathrm{IX}_{0}^{a}$ & 0,37 & 0,62 & 94 & USA., Mittelzahlen & 0,48 & 0,89 & $90-95$ \\
\hline
\end{tabular}

Wir haben absichtlich nur die höchstbelasteten amerikanischen Anlagen zum Vergleiche ausgewählt. Man erkennt sofort den beträchtlich niedrigeren Energieverbrauch unserer Versuchsanlage gegenüber den Druckluftbelüftern. Auch RIDENOUR und HENDERSON (1936, 1937), die bei strenger Parallelführung zwei Versuchsanlagen mit Druckluft- bzw. Bürstenbelüftung untersuchten, haben gezeigt, dass die letztere bei gleicher Reinigungsleistung merklich geringere Jahreskosten verursachte als die Druckluftanlage. Es wäre zweifellos wünschenswert, wenn solche Vergleiche an modernen, hochbelasteten Anlagen wiederholt werden könnten.

Als wesentlicher Nachteil der Bürstenbelïftung sind die heute noch auftretenden Schwierigkeiten bei der Konstruktion und Materialauswahl zu nennen, sobald Bürsten für hohe Dauerbelastungen (hohe Tourenzahl, 
grosse Eintauchtiefe) zu konstruieren sind. Dieses Problem ist zur Zeit noch unbefriedigend gelöst, doch könnte vorderhand ein Ausweg gefunden werden, indem die Bürstenlänge pro Kubikmeter Beckeninhalt vergrössert und dafür die Belastung der Bürsten herabgesetzt würde.

\section{d) Zusätze zum Belebtscblamm}

In Serie X wurde erstmals der Einfluss des Asbestzusatzes, den wir in früheren Versuchen bei hohen Raumbelastungen stets angewendet hatten, systematisch untersucht. Hierzu diente ein Parallelversuch mit zwei Anlagen unter vollständig identischen Bedingungen, wobei die eine mit der bis anhin üblichen Asbestdosierung betrieben wurde ( $1 \mathrm{mg} / \mathrm{l}$ Rohwasser), während die andere keinen Zusatz erhielt. In einem Unterabschnitt dieser Serie wurde dann auch die 2ofache Dosierungsmenge mit der Dosierung o verglichen. Einige Schlammabsetzkurven aus dem 2. Teil der Serie X (hohe Belastung) sind für die beiden Parallelanlagen in Abbildung I 3 dargestellt.

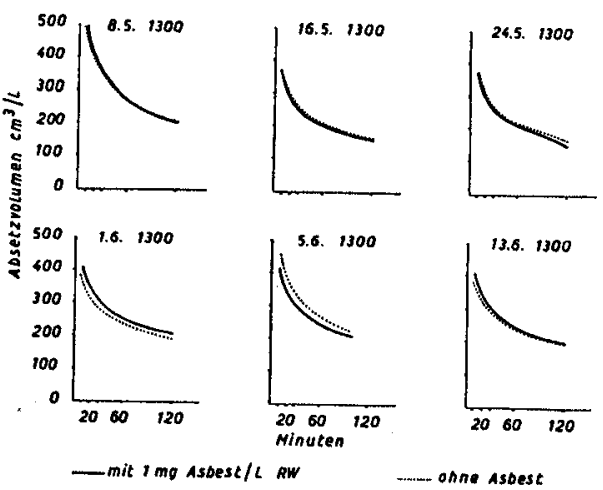

Abb. 13. Absetzkurven des Belebtschlammgemisches aus den Belüftungsbecken zweier Parallelanlagen mit und ohne Asbestzusatz ( $1 \mathrm{mg} / \mathrm{l}$ ).

Ordinaten: Schlammvolumen im Imhoff-Glas ( $\left.\mathrm{cm}^{3} / \mathrm{l}\right)$. Abszissen: Absetzzeit (min). _- Anlage mit Asbestzusatz, ……. Anlage ohne Asbestzusatz.

Die Diagramme und die Angaben in den Tabellen 9 bis 13 zeigen ohne weiteres, dass weder der Asbestzusatz von I mg/l Rohwasser noch von $20 \mathrm{mg} / \mathrm{l}$ Rohwasser die Leistung des Verfahrens gegenüber der Anlage ohne Asbestzusatz veränderte. Die Identität der Parallelversuche blieb bis in die letzten Details erbalten. Der Asbestzusatz von $20 \mathrm{mg} / \mathrm{l}$ wirkte sich auf die Dauer insofern ungünstig aus, als der Schlamm im Laufe des Versuches 
immer voluminöser wurde, da der sperrige Asbest sein Zusammensacken im Nachklärbecken verhinderte.

Auf Grund des Experimentes in Serie X haben wir die Belebtschlammanlagen nie mehr mit Asbestzusatz betrieben. Die Resultate bei der $\mathrm{Ab}$ wasserreinigung mit hohen Raumbelastungen blieben trotzdem unverändert im Rahmen der früheren Ergebnisse.

Von verschiedenen Autoren wurden Versuche mit anderen Zusätzen zum Belebtschlamm beschrieben (zum Beispiel JUNG, I939: Eisenhydroxyd; NeSMEJANOFF, 1935: Eisen- und Aluminiumsalze; HASELTINE, I93 I : Kalziumhydroxyd; ELY, I938: Lehm; MOHLMANN [mündliche Mitteilung]: Lehm, Diatomeenerde, Zellulosefasern usw.). Wie die meisten dieser Autoren kommen auch wir auf Grund diesbezüglicher Versuche zur Auffassung, dass mindestens bei der Reinigung normaler städtischer Abwässer solche Zusätze keine Verbesserung des Belebtschlammverfahrens mit sich bringen. Wir sind im Gegenteil sogar der Ansicht, dass Zusätzie anorganischer Art zum Belebtscblamm durcbaus unerwünscht sind, da sie lediglich das Schlammvolumen und den Ascbegebalt steigern, was für die nachfolgende Weiterverarbeitung des Schlammes nur nachteilig ist.

\section{BEOBAChTUNGEN AM KÜNSTLICH BeLÜFTETEN 'TROPFKÖRPER}

Beim Betriebe des Tropfkörpers haben wir bei kleinen Raumbelastungen ebenfalls keine Schwierigkeiten festgestellt. Die Wartung der Anlage konnte sich auf die Kontrolle des Luftdurchsatzes, die Reinigung verstopfter Düsen am Drehsprenger sowie das Ausbürsten von Kanälen, UUberfällen usw. beschränken. Mit steigender Raumbelastung zeigte sich aber eine zunehmende Tendenz zur Verstopfung. Dieser konnte, entgegen anderslautenden Auffassungen, mittels Steigerung der Belüftungsintensität nicht begegnet werden, sondern nur mittels Unterbruch der Tropfkörperbeschickung. Diese Beobachtung stimmt mit den Angaben von PöNNINGER (1935) überein. Auch von Hunter und Cockburn (1944) bzw. Cameron und JammeSON (1950) sind analoge Schwierigkeiten bei einem künstlich belüfteten Tropf körper beschrieben, die mittels Veränderung der Belüftungsintensität in keiner Weise bekämpft werden konnten. In unseren Serien V und VIII wurde versucht, durch eine Verlängerung der Aufenthaltszeit in der Vorklärung ein so weit behandeltes Abwasser zu erzielen, dass eine Verstopfung des Tropfkörpers nicht mehr eintreten sollte (vgl. Abschnitt I, C I). Wir erhielten aber nie den Eindruck, den Betrieb des Tropfkörpers damit wesentlich erleichtert zu haben. Wir weisen auch darauf hin, dass die 
Verstopfung nicht in erster Linie wegen einer Akkumulierung primärer Schwebestoffe in den Interstizien der Tropfkörperfüllung entstand, sondern wegen der Ausbildung eines dichten Pilzrasens in der obersten Steinschicht, also derselben Erscheinung, wie sie auch von geschlossenen und offenen Tropf körpern im Auslande beschrieben wird. Als Hauptursache für die Verstopfungen betrachten wir die zu feinkörnige oberste Tropf körperschicht. Unseres Erachtens sollte für die oberste «Verteilschicht» von $30-50 \mathrm{~cm}$ Tiefe eine Körnung von mindestens $100-120 \mathrm{~mm}$ gewählt werden.

Ein weiteres Betriebsproblem ergab sich aus den häufigen Schlammretentionen, da bei den hohen Belastungen der kontinuierliche Schlammabbau innerhalb des Tropfkörpers offenbar nicht zur Entschlammung genügte. Längere Betriebsunterbrüche, die ohnehin zwischen den Versuchsserien eingeschaltet werden mussten, wurden jeweils dazu verwendet, um mittels Spülungen, Chlorung usw. den Körper wieder zu reinigen. Auf Grund der Literaturangaben (DEKEMA, HunTER und Mitarbeiter, CAMERoN und Mitarbeiter) schliessen wir, dass zu starke Schlammansammlungen zur Hauptsache auf eine übersetzte Belastung zurückzuführen sind, denn ausländische Betriebe mit Raumbelastungen von weniger als $0,5 \mathrm{~kg} \mathrm{Bsb} b_{5} / \mathrm{m}^{3}$. Tag scheinen diesen Schwierigkeiten nicht begegnet zu sein. Änderungen der Belüftungsintensität hatten keinen Einfluss auf die Schlammabgabe des Tropfkörpers.

Betrachtet man die Sauerstoffgehalte im Abfluss des Tropfkörpers bei den verschiedenen Raumbelastungen und Lüftungsintensitäten, die wir studiert haben, so stellt man fest, dass das Abwasser im Tropfkörperabfluss stets hohe Mengen Sauerstoff enthielt. Dies galt insbesondere auch dann, wenn die Verstopfung des Körpers so weit fortgeschritten war, dass wegen des hohen Durchtrittswiderstandes die künstliche Belüftung praktisch unterbunden war.

\section{B. Entfernung von Stoffen mit biochemischem Sauerstoffbedarf}

\section{AlLGEMEINES}

Für die Dimensionierung einer biologischen Reinigungsanlage wird eine stetige Beziehung benötigt, welche, ausgehend vom gewünschten $B b_{5}$ des Anlagenabflusses und der bekannten Konzentration des Anlagenzuflusses, die zulässige Belastung des Belüftungsraumes zu berechnen gestattet. Die Raumbelastung kann entweder bezüglich der durchgesetzten Wassermenge oder bezüglich der eingebrachten Schmutzstoffmenge pro Einheit Anlagenvolumen ausgedrückt werden. Nachfolgend bezeichnen wir 
die beiden Raumbelastungen mit $R_{h}(=$ hydraulische Raumbelastung, $\mathrm{m}^{3} / \mathrm{m}^{3} \cdot \mathrm{Tag}$ ) und $R_{b}$ (= Raumbelastung bezüglich $\mathrm{Bsb}_{5}, \mathrm{~kg} \mathrm{Bsb} / \mathrm{m}^{3} \cdot \mathrm{Tag}$ ). Beide Faktoren beeinflussen die Abbauleistung einer Anlage und damit die absolute Konzentration des Anlagenabflusses. Es muss erneut darauf hingewiesen werden, dass die übliche Berechnung von Reinigungseffekten (prozentualer Anteil der entfernten Schmutzstoffe, gemessen an den zugefübrten Schmutzstoffen) ein sowohl für die Dimensionierung von Anlagen wie auch für die Praxis des Gewässerschutzes ganz untaugliches Mass darstellt, weil nur absolute Werte von Schmutzstoff konzentrationen oder Wassermengen brauchbare Rechnungsgrundlagen liefern.

Die Erstellung von Beziehungen zwischen absoluten Konzentrationen, zum Beispiel des $\mathrm{Bsb}_{5}$ in einem Anlagenabfluss und der Anlagenbelastung $\left(R_{h}\right.$ und $\left.R_{b}\right)$ stösst allgemein auf folgende Schwierigkeiten:

a) Es muss ein weiter Spielraum von Raumbelastungen und Rohwasserkonzentrationen untersucht sein, und die Anlage muss sich während der Prüfung für jede Faktorenkombination im Beharrungszustand befinden. Die vorliegenden Untersuchungen an Belebtschlammanlagen und hochbelasteten Tropfkörpern genügen unseres Erachtens diesen Forderungen.

b) Der $\Lambda$ bfluss (Zufluss zur biologischen Stufe) einer mechanischen Kläranlage einer Gemeinde unterliegt sehr grossen Konzentrationsschwankungen, sowohl innerhalb eines Tages wie auch von Tag zu Tag. Die gesuchten, stetigen Beziehungen müssen für Mittelwerte gelten, in welchen diese Schwankungen berücksichtigt sind.

c) Abwassertemperatur, Betriebsbedingungen der Reinigungsanlage und die täglichen Schwankungen der hydraulischen Raumbelastungen üben einen Einfluss auf die mittlere Qualität des Anlagenabflusses aus, der berücksichtigt werden muss.

Erste Voraussetzung für die Erstellung stetiger Beziehungen, wie sie von uns angestrebt werden, ist zunächst die Untersuchung 'von Tagesmittelproben. Damit wird die Streuung, die durch die Konzentrationsschwankungen innerhalb eines Tages entsteht, ausgeschaltet. Der Vergleich unserer Ergebnisse mit denjenigen ausländischer Anlagen wird speziell dadurch erschwert, dass sehr häufig nur Einzelproben untersucht werden, oder in vielen Fällen nicht angegeben ist, wie die untersuchten Proben gefasst worden sind. Die übrigen genannten Faktoren, welche das Verhältnis zwischen Rohwasserkonzentration und Abfluss beeinflussen, äussern sich: 
a) In der Streuung, die in Mittelwerten enthalten ist, welche innerhalb von Perioden gleichartigen Anlagebetriebes beobachtet werden (beispielsweise innerhalb von Perioden mit gleicher Abwassertemperatur);

b) in Streuungen zmischen den Mittelwerten von Untersuchungsperioden mit einem oder mehreren voneinander abweichenden Betriebsfaktoren (zum Beispiel zwischen Winter- und Sommerperioden).

Wir haben bei der Auswertung unserer. Beobachtungen mittels verschiedener statistischer Berechnungsverfahren den Einfluss der einzelnen Faktoren geprüft, um ihre Bedeutung für die Leistung einer biologischen Reinigungsanlage festzustellen, und gelangten zu nachfolgend beschriebenen Ergebnissen.

\section{BELEBTSCHLAMMVERFAHREN}

In erster Näherung ist die Konzentration des Anlagenabflusses, unter der Voraussetzung, dass immer mit denselben technischen Mitteln gearbeitet wird (Konstruktion des Belüftungsbeckens und Nachklärbeckens, Belïftungsart, Rohwasser gleichartiger Beschaffenheit bezüglich Mengenverhältnisse und Art der Schmutzstoffe), von folgenden Variablen abhängig:

hydraulische Raumbelastung und ihre Schwankung innerhalb eines Tages, Konzentration des Rohwassers, Konzentration des Belebtschlammgemisches im Belüftungsbecken, Temperatur des Rohwassers, Rücklaufschlammverhältnis und Sauerstoffspannung im Belebtschlammgemisch (Belüftungsbecken).

Von diesen Variablen sind die ersten vier von besonderer Bedeutung und weisen innerhalb unserer Versuchsreihen die grössten Schwankungen auf. Wir haben zunächst von allen Versuchsabschnitten die Abschnittsmittelwerte dieser Faktoren den Abschnittsmittelwerten der Konzentration des Anlagenabflusses gegenübergestellt. Mittels des Verfahrens der Mehrfachkorrelation wurde untersucht, welche Abhängigkeit zwischen der Abflussqualität und diesen Variablen besteht. Wir treten hier nicht auf die langwierige Rechnung ein und stellen lediglich fest:

Betrachtet man die Abschnittsmittelwerte der Bsb $_{5}$-Konzentration im Anlagenabfluss, so üben nur die bydraulische Raumbelastung und die Rohwasserkonzentration einen statistisch gesicherten Einfluss auf sie aus. Der Einfluss der Schlammkonzentration kommt nicht oder nur schwach gesichert zum Ausdruck (die Variabilität zwischen den Versuchen ist gering, 
abgesehen von vereinzelten Experimenten mit niederen Konzentrationen). Eigenartigerweise liess sich auch der Temperatureinfluss bei gesamthafter Beurteilung der Mittelwerte aller Versuche nicht herausschälen, obschon die Schlammatmung wegen der zwischen den einzelnen Versuchsabschnitten bestehenden Differenzen der mittleren Rohwassertemperaturen beträchtliche Unterschiede aufweisen musste. Diese beiden Variablen - $\mathrm{Ab}$ wassertemperatur und Schlammkonzentration im Belüftungsbecken - tragen deshalb nur zu den Streuungen innerhalb der Abschnittsmittelwerte bei, die auch noch von einer grossen Reihe anderer, zum Teil nicht einmal näher bekannter Beeinflussungen der Anlagen herrühren.

Auf Grund dieses Ergebnisses haben wir uns entschlossen, eine Beziehung zwischen Abflusskonzentration, Konzentration des Rohwassers und hydraulischer Raumbelastung $R_{h}$ mittels einer Methode aufzusuchen, deren Ergebnis alle andern Streuungen automatisch impliziert. Eine nähere Ausscheidung des Einflusses besonderer Variablen (Temperatur, Schlammkonzentration) mittels geeigneter Streuungszerlegung musste aufgegeben werden, da hierzu sogar unser umfangreiches Material nicht ausreichte.

Zunächst haben wir alle Versuche, die mit ähnlicher Raumbelastung ausgefuihrt wurden, in Gruppen zusammengefasst und erhalten:

$$
\begin{aligned}
& \text { I. } \left.R_{h} \text { bis } 5 \quad \text { (im Mittel } \sim 4,0\right) \mathrm{m}^{3} / \mathrm{m}^{3} \cdot \mathrm{Tag} \text {, } \\
& \text { II. } R_{h} \text { IO-II,2 (im Mittel I0,5) } \mathrm{m}^{3} / \mathrm{m}^{3} \cdot \mathrm{Tag} \text {, } \\
& \text { III. } R_{h} \quad \text { I5,8-17 (im Mittel I6,5) } \mathrm{m}^{3} / \mathrm{m}^{3} \cdot \mathrm{Tag} \text {, } \\
& \text { IV. } R_{h} \quad 22-22,7 \text { (im Mittel } \sim 22 \text { ) } \mathrm{m}^{3} / \mathrm{m}^{3} \cdot \text { Tag. }
\end{aligned}
$$

Sodann wurden sämtliche Einzelbeobachtungen der Rohwasserkonzentration bezüglich $\mathrm{Bsb}_{5}$ in Klassen mit der Abstufung von $20 \mathrm{mg} \mathrm{O} / \mathrm{l}$ aufgeteilt und diesen Klassen die entsprechenden Einzelmessungen der $\mathrm{Ab}-$ flusskonzentrationen gegenübergestellt. Es zeigt sich dann, dass innerhalb der Gruppen mit gleicher Raumbelastung eine lineare Regression zwischen der Konzentration des Rohwassers und des Abflusses besteht. Die Streuungen um die Regressionslinie enthalten sämtliche nichtberücksichtigten Variablen. Die Regressionskoeffizienten gelten allerdings nur für den Bereich der wirklich beobachteten Rohwasserkonzentrationen. Eine Fortsetzung der Regressionsgeraden speziell gegen niedrigere als die beobachteten Rohwasserkonzentrationen, ist nicht erlaubt, während eine Extrapolation gegen höhere Konzentrationen in beschränktem Umfange gestattet ist.

Auf Grund der gefundenen Regressionen lassen sich nun für verschiedene Rohwässer Kurven aufzeichnen, welche die Abhängigkeit der Ab- 
flusskonzentration von der hydraulischen Raumbelastung darstellen (Abb. I4). In dieser Figur wurden die Schlammkonzentrationen, die in den einzelnen Experimenten im Belüftungsraum gehalten wurden, in einem besonderen Diagramm ebenfalls eingetragen. Diese letzteren Angaben zeigen nochmals, dass, abgesehen von wenigen Ausnahmen, im Belastungsbereich über Io $\mathrm{m}^{3} / \mathrm{m}^{3}$. Tag mit Schlammengen von $3000 \mathrm{mg} / \mathrm{l}$ und mehr
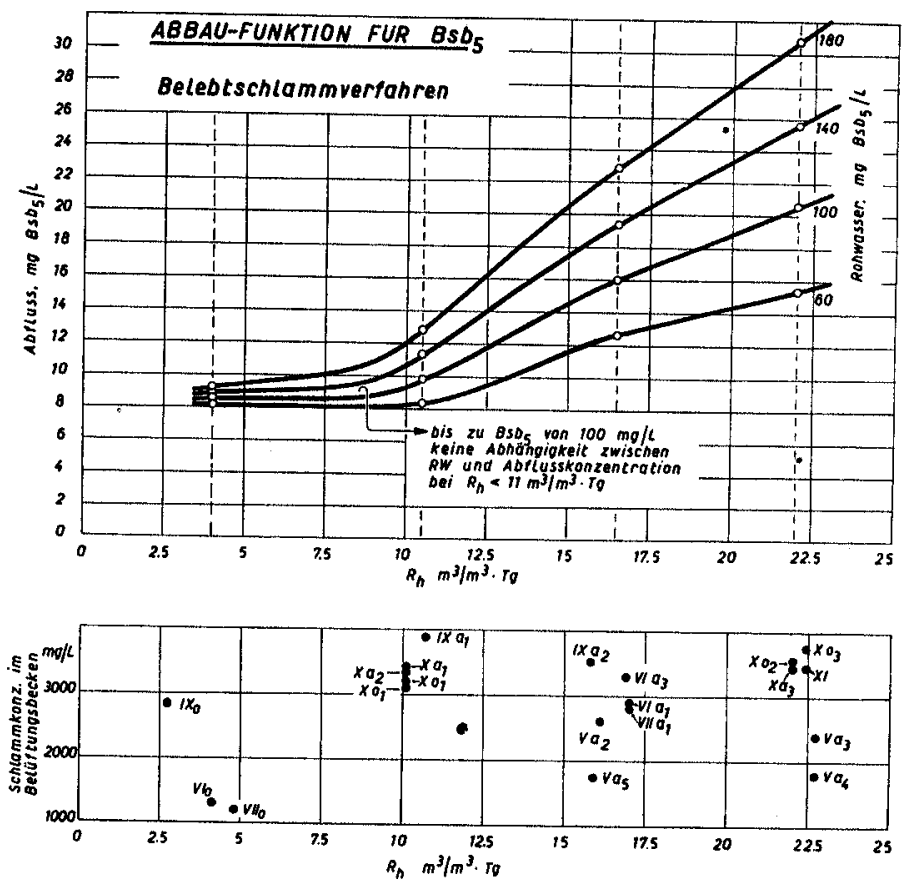

Abb. 14. Wahrscheinlichste $\mathrm{Bsb}_{5}$-Werte des Abflusses der Belebtschlammanlage in Funktion des $\mathrm{Bsb}_{5}$ des zufliessenden Rohwassers und der hydraulischen Raumbelastung.

Ordinate: $\mathrm{Bsb}_{5} \mathrm{im}$ Abfluss (mg O/l). Abszisse: Hydraulische Raumbelastung $\left(\mathrm{m}^{3} / \mathrm{m}^{3} \cdot \mathrm{Tag}\right.$ ). Parameter: $\mathrm{Bsb}_{\mathbf{5}}$ im Rohwasser (mg O/l).

Unterer Streifen: Mittlere Schlammgehalte im Belüftungsbecken bei den Experimenten, die als Grundlage für die Kurvenschar dienten.

Ordinate: Schlammgehalt (mg Trockensubstanz/l). Abszisse: Hydraulische Raumbelastung $\left(\mathrm{m}^{3} / \mathrm{m}^{3} \cdot \mathrm{Tag}\right)$.

gearbeitet wurde. Wir schreiben die erzielten hohen Abbauleistungen in unseren Versuchen ausschliesslich dieser Verfahrenstechnik zu, oder, mit anderen Worten, unsere Kurvenzüge gelten nur dann, wenn die Anlage bei Belastungen von $R_{h}>$ Io $\mathrm{m}^{3} / \mathrm{m}^{3} \cdot$ Tag bzw. $R_{b}>\mathrm{I}, 2$ bis $\mathrm{I}, 4 \mathrm{~kg}$ $\mathrm{Bsb}_{5} / \mathrm{m}^{3}$. Tag mit einer Belebtschlammkonzentration von mindestens $3000 \mathrm{mg} / \mathrm{l}$ betrieben wird. 
Es zeigt sich, dass bis zu Raumbelastungen von $10 \mathrm{~m}^{3} / \mathrm{m}^{3}$. Tag ein verhältnismässig geringer Einfluss der Rohwasserkonzentration auf die Abflussqualität auftritt und mit zunehmender Raumbelastung und zunehmender Rohwasserkonzentration der $\mathrm{Bsb}_{5}$ des Abflusses ansteigt. Es ist bemerkenswert, dass bei Raumbelastungen von $\sim 22 \mathrm{~m}^{3} / \mathrm{m}^{3} \cdot$ Tag und der Tagesmittelkonzentration von $\mathrm{I} 80 \mathrm{mg} \mathrm{Bsb}_{5} / \mathrm{I}$ die Abflüsse im Mittel höchstens 30-32 mg Bsb $/$ l enthalten.

Unser Diagramm erhebt nicht Anspruch auf eine sehr hohe Genauigkeit, da zu viele Faktoren, die auf den Reinigungseffekt einer Belebtschlammanlage einwirken, nicht berücksichtigt werden konnten. Die Darstellung gibt lediglich die wabrscbeinlichste Bezichung zwischen den Tagesmittelwerten des $\mathrm{Bsb}_{5}$ von $\mathrm{Zu}$ - und Abfluss der Anlage an. Wir weisen ferner nochmals ausdrücklich darauf hin, dass sich die Kurvenschar auf Tagesmittelmerten aufbaut und nicht auf Durchschnitten ganzer Versuchsperioden. Immerhin ergibt eine Kontrolle sofort, dass sich auch die Durchschnittswerte ganzer Versuchsabschnitte für Rohwasser- und Abflusskonzentrationen mit befriedigender Genauigkeit in die Kurvenschar hineinpassen, denn der grössere Teil der gefundenen mittleren Bsb $_{5}$-Werte der Anlagenabflüsse entspricht ziemlich genau den vom Diagramm geforderten Konzentrationen oder ist sogar zum Teil nicht unwesentlich niedriger.

Es ist schwierig, unsere Ergebnisse, die an einer Anlage unter praktischen Betriebsbedingungen und im technischen Maßstabe gewonnen wurden, mit Angaben anderer Autoren zu vergleichen, da die uns bekannten systematischen Reihenversuche mit dem Belebtschlammverfahren lediglich in Laboratoriumsanlagen erfolgten. GREELEY (1943) veröffentlichte eine Beziehung zwischen Belastung und Abbauleistung von Belebtschlammanlagen, die in Abbildung I5 eingetragen ist. In die gleiche Figur haben wir auch die von OKUN (1949) bei seinen Versuchen mit Belüftung mittels reinem Sauerstoff gefundene Funktion eingetragen. Die Darstellungsweise beider Autoren hat den Nachteil, dass die hydraulische Raumbelastung der Anlagen nur implizite zum Ausdruck kommt. Diese spielt aber schon für sich allein eine wichtige Rolle für das Endergebnis der Abbauleistung, wie wir dies in Abbildung I4 zeigen konnten. Um dennoch einen Vergleich zu ermöglichen, haben wir in Abbildung $\mathrm{I}_{5}$ die unseren Beobachtungen entsprechenden Beziehungen. zwischen der Schmutzstoffbelastung $R_{b}$ und der Abbauleistung für zwei Rohwasserkonzentrationen eingetragen (Grundlage: Kurven in Abb.I4). Ferner setzten wir die Einzelergebnisse von Mittelwerten aus denjenigen Ver- 
suchsabschnitten ein, bei denen die Rohwasserkonzentration im Bereiche zwischen rund I IO-I80 mg Bsb $/ 1$ lag. Es ergibt sich aus der Abbildung, dass unsere Experimente bedeutend höhere Abbauleistungen des Belebtschlammverfahrens aufweisen als nach GREELEY zu erwarten wären. Sie nähern sich zum Teil weitgehend den Ergebnissen von OkUN, die unter ganz anderen (vermutlich viel günstigeren) Bedingungen gewonnen wurden. Eine weitere Reihenuntersuchung mit verschiedenen Anlagenbelastungen stammt von SMITH (I952). Er hat seine Beobachtungen zusammen mit Betriebsergebnissen amerikanischer Grossanlagen gemäss

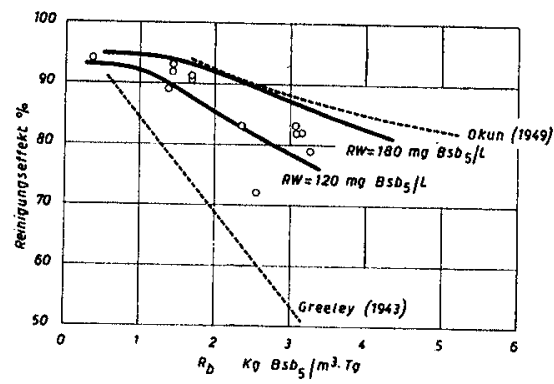

Abb. 15. Reinigungseffekte in Funktion der Raumbelastung mit Bsb $_{5}$ bei Belebtschlammanlagen nach GREeley (1943), OKUN (1949) und bei unseren Versuchen. $O=$ Mittelwerte unserer Versuche bei Rohwasserkonzentrationen zwischen 110 und $150 \mathrm{mg} / 1$. Ordinate: Reinigungseffelt (\%). Abszisse: Raumbelastung mit $\mathrm{Bsb}_{5}\left(\mathrm{~kg} \mathrm{Bsb} \mathrm{B}_{5} / \mathrm{m}^{3} \cdot \mathrm{Tag}\right)$.

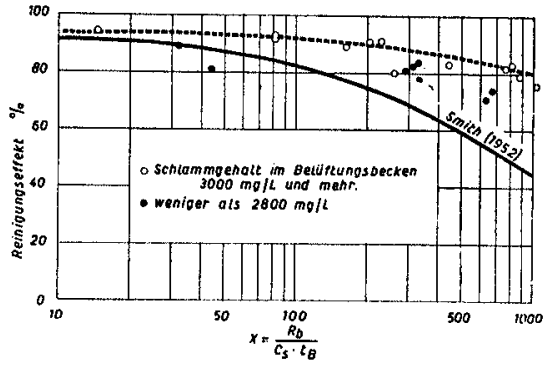

Abb. 16. Reinigungseffelst in Funktion von Raumbelastung mit $\mathrm{Bsb}_{5}$, Schlammkonzentration im Belüftungsraum und Belüftungszeit bei Belebtschlammanlagen nach Sмгтн (1952) und bei unseren Versuchen. Ordinate: Reinigungseffekt (\%). Abszisse: (Belastungs" parametern (vgl. Text).

einem Vorschlag von Farr und Thomas (1950) in Funktion eines «Belastungsparameters» ausgedrückt, der die Raumbelastung mit $\mathrm{Bsb}_{5}$, die Schlammkonzentration im Belüftungsbecken und die hydraulische Raumbelastung (als Belüftungszeit) einschliesst. Der Belastungsparameter hat die Form:

$$
X=\frac{R_{b}}{C_{s} t_{B}} \cdot \mathrm{IOOO},
$$

und für den Reinigungseffekt $\eta$ wurde die Beziehung

$$
\eta=\frac{100}{1+0,00266(\bar{X}+45)^{0,88}}
$$

gefunden. 
Darin bedeutet:

$R_{b}$ Raumbelastung mit $\mathrm{Bsb}_{5}, \mathrm{~kg} \mathrm{Bsb} / \mathrm{m}^{3} \cdot \mathrm{Tag}$,

$C_{s}$ Schlammkonzentration im Belüftungsbecken, $\mathrm{kg}$ Trockensubstanz $/ \mathrm{m}^{3}$, $t_{B}$ Belüftungszeit (Rohwasser allein), h,

$X$ «Belastungsparameter»,

$\eta$ Reinigungseffekt in Prozent.

In Abbildung I 6 ist die vorstehende Funktion aufgezeichnet, und zugleich wurden die entsprechenden Durchschnittswerte unserer Experimente eingetragen. Es zeigt sich zunächst, dass alle unsere Versuche wesentlich höhere Reinigungseffekte zeitigten, als dies der Funktion von SMrTH entspricht. Ferner ergibt sich die interessante Tatsache, dass sich unsere Experimente deutlich in zwei Gruppen scheiden lassen:

I. Versuche, bei denen die Schlammkonzentration im Belüftungsbecken mindestens $3000 \mathrm{mg} / \mathrm{l}$ betrug. Die Leistungen der Anlagen lassen sich (mit einer Ausnahme) mit guter Annäherung durch eine Kurve verbinden, die für die höchsten Werte von $X$ bis zu $35 \%$ höhere Reinigungseffekte aufzeigt, als sie in den Versuchen von SMITH beobachtet wurden.

2. Versuche mit geringen Schlammkonzentrationen. Die Ergebnisse dieser Gruppe fallen deutlich ab gegenüber den vorher geschilderten, sind aber immer noch besser als sie gemäss der Funktion von SMITH erwartet werden könnten.

Es ist an sich nicht überraschend, dass die von OKun und SMITH aut Grund von Laboratoriumsexperimenten aufgestellten Beziehungen zwischen Belastung und Abbauleistung nicht mit unseren Ergebnissen übereinstimmen; hierzu ist die Maßstabsdifferenz zwischen den untersuchten Anlagen viel zu gross. Es wäre jedoch zu erwarten gewesen, dass die Modellversuche im kleinen Maßstab wie üblich zu günstigeren Resultaten führen würden als ein technischer Betrieb. Wie wir schon früher ausführten, möchten wir unsere relativ günstigen Betriebsergebnisse lediglich einer konsequenten Durchführung maximaler Belastungen unserer Belüftungsanlage mit Schlamm bzw. maximaler Belastungen des Schlammes mit Abwasser zuschreiben. Umgekehrt ergibt sich hieraus, dass die konventionelle Betriebsweise von Belebtschlammanlagen die Belastbarkeit des Verfahrens offenbar bei weitem nicht ausnützt. Dies gilt unseres Erachtens auch für die von IMHOFF in der Ausgabe I95I des Taschenbuches angegebenen maximalen Raumbelastungen -von- $\mathrm{I}, 4 \mathrm{~kg} \mathrm{Bsb}{ }_{5} / \mathrm{m}^{3}$. Tag für sogenannte Teilreinigung nach dem Belebtschlammverfahren. Wir sind der 
Auffassung, dass auf Grund unserer Versuche diese Belastung ohne weiteres angewendet werden darf, wenn sogenannte «Vollreinigung» des Abwassers verlangt wird. Gemäss unserem Diagramm Abbildung I5 würde nämlich bei einem vorgeklärten Rohwasser mit dem $\mathrm{Bsb}_{5}$ von $\mathrm{I} 8 \mathrm{o} \mathrm{mg/l}$ ein Abfluss mit II-12 mg Bsb $/ 1$ zu erwarten sein. Bei einem Rohwasser mit I $40 \mathrm{mg} \mathrm{Bsb} / \mathrm{l}$ würde die Konzentration des Abflusses um Io-II mg $\mathrm{Bsb}_{5} / \mathrm{l}$ betragen. Derart weitgehende Abwasserbehandlungen ergeben nach unseren früheren Untersuchungen in Vorflutermodellen (WUHRMANN, I95I) Anlagenabflüsse, die praktisch kein Verdünnungswasser mehr in einer Vorflut benötigen, um Abwasserschäden zu verhindern.

Es ist bemerkenswert, dass unsere Versuche auch zu viel besseren Resultaten führten als diejenigen von SETTER (I944) in der Anlage Jamaica in New York. SetTER betrieb diese Anlage mit einem an primären Schwebestoffen sehr reichen Abwasser (keine Vorklärung!) bei einer Belastung von 0,6 bis $\mathrm{I}, \mathrm{I} 2 \mathrm{~kg} \mathrm{Bsb} / 5 / \mathrm{m}^{3}$. Tag und erreichte Reinigungseffekte zwischen 66 und $77 \%$. Dabei ist noch zu berücksichtigen, dass bei jenem Rohwasser ein wesentlicher Teil des $\mathrm{Bsb}_{5}$ in primären, absetzbaren Schwebestoffen investiert war und somit die Belüftungsanlage kaum belastete. Wir sehen auf Grund unserer Erfahrungen die Ursache für diesen schlechten Wirkungsgrad der Jamaica-Anlage einzig in der viel zu geringen Konzentration des Belebtschlammes in den Belüftungsbecken (es wurden 500-800 mg Schlamm/1 geführt), worauf wir schon früher aufmerksam machten (WUHRMANN, 1948).

In diesem Zusammenhang ist auch noch auf das von ULLRICH und SMITH (195I) veröffentlichte "Biosorption»-Verfahren hinzuweisen. Die beiden Autoren kehren zum Wiederbelüftungssystem für Belebtschlamm zurück, wobei lediglich eine kurze Kontaktzeit zwischen Rohwasser und Belebtschlamm erfolgt. Mit einem nicht vorgeklärten Rohwasser von der Konzentration $264 \mathrm{mg} \mathrm{Bsb} / 1$ (Mittelwert) erreichten sie einen Abfluss mit $20 \mathrm{mg} \mathrm{Bsb} b_{5} / \mathrm{l}$. Das verwendete Gesamtvolumen für die Wiederbelüftungskammer und die Mischkammer wurde mit einer Raumbelastung von rund $8 \mathrm{~m}^{3} / \mathrm{m}^{3 .}$.Tag ausgenützt. Unter diesen Umständen wären nach unseren Untersuchungen bei der üblichen Betriebsweise unserer Anlagen Abflüsse mit rund $\mathrm{I} 3 \mathrm{mg} \mathrm{Bsb} / \mathrm{l}$ zu erwarten, wobei es allerdings sehr fraglich ist, ob eine so weitgehende Extrapolation vorgenommen werden kann. Wir möchten uns deshalb einer weitergehenden Beurteilung des «Biosorption»-Verfahrens enthalten, bis zusätzliche Erfahrungen und eingehende Vergleichsuntersuchungen vorliegen. 


\section{KÜNSTLICH BELÜFTETER TROPFKÖRPER}

Beim künstlich belüfteten Tropfkörper sind die hauptsächlichsten Variablen, die bei gegebenem Bauwerk die Abflussqualität beeinflussen, wiederum die Rohwasserkonzentration, die hydraulische Raumbelastung und die Rohwassertemperatur.

Ferner ist die Beschickungsweise des Tropfkörpers, das heisst der tägliche oder periodische Wechsel der hydraulischen Raumbelastung auf Grund der Versuche von Pönninger von grosser Bedeutung. Da die meisten der ausgewerteten Tropfkörperversuche mit wechselnder Beschickung (Maximum:Minimum $=2: \mathrm{I}$ bis $5: \mathrm{I}$ ) durchgeführt wurden, enthalten unsere nachfolgenden Ergebnisse bereits diese wichtige Betriebsvoraussetzung. Dagegen haben wir eindeutig festgestellt, dass die Menge künstlich zugeführter Luft keine messbare Rolle spielt, worauf wir noch zu sprechen kommen werden.

Mittels des gleichen statistischen Verfahrens wie bei der Auswertung der Belebtschlammversuche haben wir die Einzelmessungen des $B_{s} b_{5}$ am Tropf körper miteinander in Beziehung gesetzt, wobei vier Raumbelastungsgruppen aus den Versuchen gebildet wurden. Diese Ergebnisse lassen wiederum stetige Beziehungen zwischen hydraulischer Raumbelastung und Abflussqualität bei verschiedenen Rohwasserkonzentrationen erkennen (Abb. I7). Als charakteristisches Merkmal der entstehenden Kurvenschar ist die sprunghafte Verscblecbterung des Abflusses der Tropfkörperanlage (inkl. Nacbklärbecken) bei Raumbelastungen über $5 \mathrm{~m}^{3} / \mathrm{m}^{3} \cdot$ Tag und Robmasserkonzentrationen über $60 \mathrm{mg} B s b_{5} / l$ hervorzuheben.

Wir haben aus der Untersuchung künstlich belüfteter Tropfkörper von PönNINGER (I938) die Funktion zwischen Raumbelastung und Abflussqualität ebenfalls in unsere Figur eingetragen, wobei diese Kurven für Rohwasser der mittleren Konzentration von schätzungsweise $\sim 300 \mathrm{mg} \mathrm{Bsb}_{5} / \mathrm{l}$ gelten (vgl. Figur I 7 bei PöNNINGER). Ferner wurde für diese Rohwasserkonzentration die Belastungsfunktion auf Grund einer Extrapolation unserer Regressionslinien berechnet und zum Vergleiche eingezeichnet. Es zeigt sich, dass die Kurven von PöNNINGER den gleichen grundsätzlichen Verlauf besitzen wie unsere eigenen, der steile Aufstieg der Abflusskonzentration bei ihm jedoch schon bei geringeren Raumbelastungen eintritt, als dies auf Grund unserer Beobachtungen zu erwarten wäre. Diese Tatsache ist besonders deshalb bemerkenswert, weil in den Versuchen PönNingers der Tropfkörper täglich während einiger Stunden sowie periodisch während ganzer Tage nicht beschickt wurde, eine Betriebs- 
massnahme, die PöNNINGER als unerlässlich für eine gute Dauerleistung des Tropfkörpers betrachtet. In unseren Versuchen dagegen erfolgte die Abwasserzufuhr ununterbrochen während $24 \mathrm{~h}$.

Da gelegentlich immer noch die Auffassung vertreten wird, dass künstlich belüftete Tropf körper höhere Abbauleistungen erreichen könnten als gewöhnliche offene Filter, scheint es uns angezeigt, auf Grund unserer Erfahrungen und an Hand ausländischer Beobachtungen grundsätzlich die

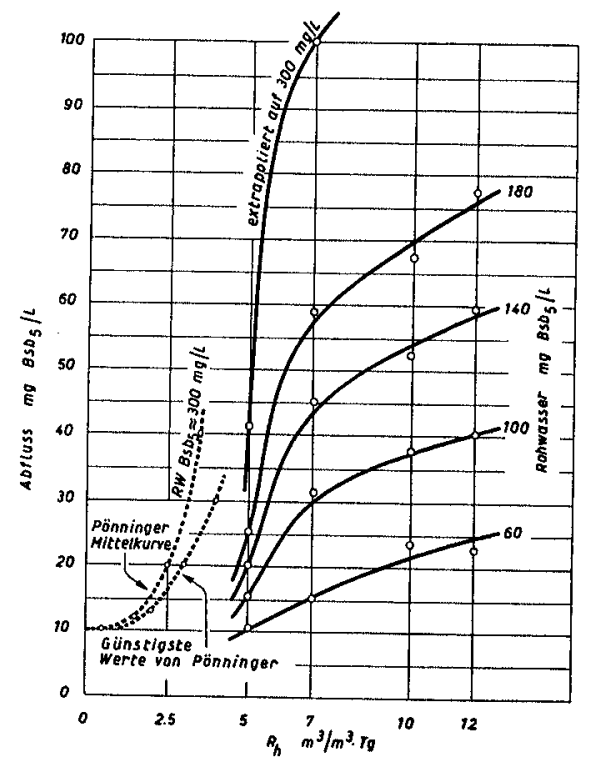

Abb. 17. Wahrscheinlichste $\mathrm{Bsb}_{5}$-Werte des Abflusses des künstlich belüfteten Tropfkörpers in Funktion des $\mathrm{Bsb}_{5}$ des zufliessenden Rohwassers und der hydraulischen Raumbelastung. Die Kurve für Rohwasser $=300 \mathrm{mg} / 1$ ist extrapoliert und den Werten von PönNınGER (1938) gegenübergestellt.

Ordinate: $\mathrm{Bsb}_{5}$ im Abfluss (mg O/1). Abszisse: Hydraulische Raumbelastung $\left(\mathrm{m}^{3} / \mathrm{m}^{3} \cdot \mathrm{Tag}\right.$ ). Parameter: $\mathrm{Bsb}_{5}$ im Rohwasser (mg O/1).

Frage zu prüfen, ob Belege für diesen Vorteil vorhanden sind. Im Hinblick auf die hohen zusätzlichen Kosten, welche die Überdeckung von Tropfkörpern (speziell bei grossen Durchmessern) verursacht, ist diese Frage in wirtschaftlicher Hinsicht bedeutungsvoll.

Geht man von einem Rohwasser bestimmter Konzentration und Temperatur aus, so hängt die Qualität des Tropf körperabflusses (nach Nachklärung) in erster Linie von folgenden Faktoren $a b$ : 
a) Von Art und Menge der abbauenden Organismenmasse, mit der das durchsickernde Abwasser in der Tropfkörperfüllung in Berührung kommt;

b) von der Kontaktzeit zwischen Organismenfilm und Abwasser;

c) von der Menge des wegen Kurzschlüssen unverändert den Tropfkörper durchlaufenden Rohwassers.

Vorausgesetzt ist dabei, dass Sauerstoff in den Hohlräumen der Tropfkörpermasse stets vorhanden ist. Die Höhe und der Körnungsgrad der Füllung sowie die Raumbelastung $R_{b}$ sind in vorstehenden Bedingungen impliziert. TomLINSON (1950) hat an Modellkörpern systematisch die Bedeutung der Raumbelastung $R_{b}$ für die Abbauleistung bei gegebenem Tropf körperaufbau untersucht und gelangt zu ganz ähnlichen Diagrammen, wie sie in Abbildung I8 für die Darstellung unserer Ergebnisse aufgestellt wurden. Seine Resultate sind allerdings wesentlich ungünstiger als die unsrigen, wenigstens soweit gleiche Bereiche von Raumbelastungen geprüft wurden. Dies ist jedoch auf die niedere Füllungshöhe der Filterbeete zurückzuführen, die TomLINson zur Verfügung standen $(\mathrm{I}, 8 \mathrm{~m})$. Die Körnung des Füllmaterials war jedoch in der Hauptmasse ebenso fein wie bei unserm Filter. Aus dem Vergleich mit diesen Untersuchungen ergibt sich somit, dass bei böberer Füllung zmeifellos eine Verbesserung der Leistung bei einmaligem Abmasserdurchgang erzielt wird. Eine gleichartige Beobachtung ergibt sich auch aus den Vergleichsversuchen von DEKEMA und Murray (1942), die Körper von der Füllhöhe r, $8 \mathrm{~m}$ und 3,6 m (letzterer mit künstlicher Belüftung) prüften. Dagegen haben die Versuche von TomLınson gezeigt, dass die Korngrösse des Füllmaterials wenigstens innerhalb des untersuchten Bereiches und bei den geprüften Belastungen keine erkennbare Rolle für das Ausmass der Reinigung spielt.

Die bei der Schaffung des künstlich belüfteten Tropf körpers wegleitende Idee, mittels grosser Füllhöhe eine Leistungssteigerung zu erreichen, hat sich somit als richtig erwiesen. Dass der dem System ebenfalls zugrunde gelegte Gedanke, mit kleiner Korngrösse des Füllmaterials die Abwasserreinigung zu verbessern, richtig ist, konnte dagegen nicht bewiesen werden (jedenfalls nicht bei Korngrössen zwischen 20 und $75 \mathrm{~mm}$ ).

Damit erhebt sich die Frage: Ist es notwendig, hochgefüllte Tropfkörper künstlich zu belüften, um eine normale Tätigkeit seiner Biozönosen zu gewährleisten? Wir haben unseren Tropf körper nie bei längerem Unterbruch der künstlichen Belüftung untersucht. Immerhin konnten wir in keinem Versuche eine Abhängigkeit der Abbauleistung von der Intensität der künstlichen Belüftung nachweisen. Auch zeigte der Tropfkörperabfluss stets Sauerstoffgehalte von mindestens $4,5 \mathrm{mg} \mathrm{O} / \mathrm{l}$, unabhängig 
vom Luftdurchsatz. Dagegen liegen von ausländischen Grossanlagen einige einwandfreie Vergleichsuntersuchungen vor, welche die gestellte Frage klar beantworten lassen. CAMERON und JAMIESON (I950) berichten über Untersuchungen, bei denen ein und derselbe Filter [Füllhöhe $5,4 \mathrm{~m}(!)$, Körnung der Hauptmasse $35 / 50 \mathrm{~mm}$ ] mit und ohne künstliche Belüftung während langer Zeit betrieben wurde. Sie stellten keinerlei Vorteil der künstlichen Belüftung fest. VosLOo und O'REILLY (I945) konnten zwei identisch gebaute und mit demselben Abwasser bei gleichen Raumbelastungen betriebene Tropf körper von 3,6 $\mathrm{m}$ Füllhöhe und der Körnung $35 / 50 \mathrm{~mm}$ über mehrere Jahre vergleichen. Sie kommen zum Schluss, dass die künstliche Belüftung in der warmen Jahreszeit ohne Einfluss auf den Reinigungseffekt ist, im Winter dagegen nachteilig wirkt, weil eine fühlbare Abkühlung des durchrieselnden Abwassers eintreten soll. An Hand von Analysenresultaten, die als Stichproben von ROHDE (I95I, I952) von den Kläranlagen Arnsberg (künstlich belüftete Tropfkörper, $\mathrm{H}=4 \mathrm{~m}$ ), und Hagen (offene Tropf körper, $\mathrm{H}=3, \mathrm{I} 5 \mathrm{~m}$ ) veröffentlicht wurden, ergibt sich ebenfalls, dass kein Unterschied in der Leistung der beiden Anlagen besteht, der auf die künstliche Belüftung zurückgeführt werden könnte. Die Abbauleistungen beider Anlagen liegen übrigens teilweise erheblich über den Werten, die wir bei unseren Untersuchungen messen konnten (eine nähere Prüfung dieser Differenz ist uns leider nicht möglich, da ROHDE nur einige Einzelwerte angegeben hat). Auch den Angaben von BLUNK (1939) über die Kläranlage Soest sowie denen von HUSMANN und LANZ (1938) über einen kleinen Versuchstropfkörper der Stadt Zürich kann entnommen werden, dass die künstliche Belüftung ohne Einfluss auf die Reinigungsleistung der Körper war (die von HUSMANN beschriebene Abnahme der Nitrifikation nach Ausschalten der künstlichen Belüftung kann nicht als Abnahme der Tropfkörperleistung bezeichnet werden). Es ergibt sich somit eindeutig, dass die hohe Füllung eines Tropfkörpers (auch wenn ein feinkörniges Füllmaterial verwendet wird) eine künstliche Belüftung und damit eine luftdichte Filterüberdeckung nicbt erfordert, und zwar weder zur Erhaltung eines gleichmässigen Betriebes (Verhinderung von Verstopfung) noch zur Leistungssteigerung. Wir stimmen damit ganz mit der Auffassung überein, die IMHOFF bereits 1949 darlegte. Auch zum Schutze gegen Insektenausflug scheint uns eine Überdeckung bei grösseren Anlagen, die einen relativ ausgeglichenen Abwasserzufluss besitzen und somit immer mit hoher $R_{h}$ betrieben werden können, unnötig zu sein. Körper von $3 \mathrm{~m}$ Füllhöhe besitzen bei $R_{h}=5 \mathrm{~m}^{3} / \mathrm{m}^{3} \cdot \mathrm{Tag}$ eine Oberflächenbelastung von is $\mathrm{m} / \mathrm{Tag}$, bei $R_{h}=3 \mathrm{~m}^{3} / \mathrm{m}^{3}$. Tag eine 
solche von $9 \mathrm{~m} / \mathrm{Tag}$. Innerhalb des Bereiches einer Raumbelastung, wie wir sie bei mittleren Rohwasserkonzentrationen als zulässig betrachten, kommt man somit zu Oberflächenbelastungen, die einen Insektenausflug weitgehend verhindern, wie dies ToMLINson (1945) und andere Beobachter deutlich nachweisen konnten. Anders liegen die Verhältnisse bei Kleinanlagen mit ihrem im allgemeinen sehr unregelmässigen Abwasserzufluss. In solchen Fällen scheint uns in unmittelbarer Nähe von. Wohngebäuden eine Abdeckung von Tropf körpern notwendig. Doch ist auch dann eine künstliche Belüftung mittels Ventilatoren entbehrlich, da vergitterte Öffnungen in der Überdeckung für einen genügenden Luftaustausch vollständig ausreichen. Man wird sich auch vielerorts fragen müssen, ob die mit hoher Tropfkörperfüllung erreichte Leistungssteigerung nicht vorteilhafterweise durch die Konstruktion von zwei niedrigeren Filtern, die hintereinandergeschaltet sind, erhalten wird. Die Untersuchungen der englischen Schule weisen klar darauf hin, dass diese sogenannte Doppelfiltration bessere Resultate zeitigt als ein einmaliger Tropf körperdurchgang, da Kurzschlüsse, die einen wesentlichen Einfluss auf die Abflussqualität ausüben, damit stark vermindert werden. Es scheint auch, dass dieses System bessere Leistungen hervorbringt als die Tropfkörper mit Rückspülung. Strenge Vergleichsuntersuchungen über diese Frage wären zweifellos yon grossem Interesse.

Es erübrigt sich, einen ausführlichen Vergleich unserer Ergebnisse an der Belebtschlamm- und Tropfkörperanlage bezüglich des $B b_{5}$-Abbaues anzustellen, da die beiden Abbildungen I 5 und I 6 alle Angaben vermitteln. Es sei nur als Einzelheit herausgestellt, dass bei der zum Beispiel in Zürich vorhandenen mittleren $A$ bwasserkonzentration von rund $\mathrm{I}_{4} \mathrm{O} \mathrm{mg} \mathrm{Bs}_{5} / \mathrm{l}$ und bei Voraussetzung einer Abflusskonzentration der biologischen Stufe von $25 \mathrm{mg} \mathrm{Bs} b_{5} / 1$ der Tropfkörper mit einer hydraulischen Raumbelastung von $5-6 \mathrm{~m}^{3} / \mathrm{m}^{3} \cdot$ Tag, eine Belebtschlammanlage dagegen mit rund $2 \mathrm{I} \mathrm{m}^{3} / \mathrm{m}^{3} \cdot \mathrm{Tag}$ betrieben werden muss. Es steht also in diesem Falle gleichwertigen $\mathrm{Ab}$ flüssen ein Verhältnis der Bauvolumina von rund 3,8: I für die biologische Stufe gegenüber (Nachklärbecken nicht eingerechnet). Berücksichtigt man noch die höheren Ansprüche eines Tropf körperbetriebes an die Vorklärung des Rohwassers im Vergleich mit Belebtschlammanlagen, so fallen die benötigten Grössen der Anlageteile bei den beiden Verfahren noch mehr auseinander. Immerhin ist eine gewisse Kompensation zugunsten der Tropfkörper bei den Volumina der Faulräume zu erwarten, da die Schlammproduktion eines Filterbeetes gemäss Abschnitt III, E, wesentlich geringer als diejenige einer Belebtschlammanlage ist. 


\section{G. Umsatz der Stickstoffverbindungen}

Es sind folgende Gesichtspunkte von praktischem Interesse, welche bezüglich der Stickstoffkomponenten beim Vergleich des Zu- bzw. Abflusses einer biologischen Reinigungsanlage diskutiert werden müssen:

I. Veränderung des Gehaltes an organischen Stickstoffverbindungen, die noch als Nähr- und Energiesubstrate für beterotropbe Organismen in Betracht kommen.

2. Veränderung des Totalgebaltes an N-Verbindungen bei der Abwasserreinigung ( $N$-Elimination).

3. Veränderung der Mengenverhältnisse einzelner N-Komponenten bei der Abwasserreinigung.

4. Gesamt-N-Bilanz einer Abwasserreinigungsanlage.

Genauere, systematische Untersuchungen über den Stickstoffumsatz von biologischen Abwasserreinigungsanlagen technischer Dimensionen sind in der Literatur nicht zahlreich. Häufig werden in der Praxis nur das Ammonium, Nitrate und Nitrite regelmässig gemessen, da an Hand dieser Verbindungen die Arbeitsweise der Anlagen geprïft wird. Im Hinblick auf den Schutz der Gewässer vor Massenentwicklungen beterotropher Organismen ist jedoch der Gehalt der Abwässer an organischen Stickstoffverbindungen massgebend, und zur Beurteilung der Eutrophiegefahr muss die Konzentration des Gesamtstickstoffes (unabhängig von der Bindungsform) bekannt sein.

Aus den seit 1946 systematisch geprüften Stickstoffbilanzen unserer Versuchsanlagen haben wir versucht, einerseits über die Umwandlung der Stickstoffverbindungen bei der Abwasserreinigung und anderseits über die quantitativen Verhältnisse der Stickstoff bindang im Schlamm der Reinigungsanlagen Aufschluss zu erlangen.

\section{UMWANDLUNG DER STICKSTOFFVERBINDUNGEN}

Das zürcherische Abwasser (nach Vorklärung) ist dadurch gekennzeichnet, dass sein Gehalt an Gesamtstickstoff (rund $30 \mathrm{mg} / \mathrm{l}$ ) stets zu rund $30-32 \%$ aus organischem, $65-68 \%$ aus Ammoniak- und $2-4 \%$ aus Nitrat- und Nitritstickstoff zusammengesetzt ist. Unter Ammoniakstickstoff reihen wir auch den im Rohwasser noch vorliegenden, unzersetzten Harnstoffstickstoff ein. Von diesen N-Verbindungen, das heisst zur Hauptsache vom organischen Stickstoff, sind rund $30 \%$ in den primären Schwebe- 
stoffen des abgesetzten Rohwassers gebunden, und die Sterilfiltration des unbehandelten Abwassers bringt deshalb von vornherein eine N-Elimination von rund $30 \%$ zustande.

Die Behandlung des Rohwassers in einer biologischen Reinigungsanlage soll neben den stickstoff haltigen, festen Stoffen in erster Linie die gelösten, organischen N-Verbindungen aus dem Abwasser zum Verschwinden bringen. Je nach Betriebsweise kann auch der Ammoniakstickstoff zu Nitrat oxydiert werden, wie dies in Vollreinigungsanlagen vielfach noch üblich ist. Wir stellen, in Übereinstimmung mit andern Autoren, ausdrücklich fest, dass die letztere Reaktion für den Gewässerschutz unnötig ist und lediglich zu erhöhten Betriebskosten führt.

Bei allen Versuchen mit bocbbelasteten Belebungsanlagen haben wir festgestellt, dass der Gehalt des Rohwassers an organischem Stickstoff in ähnlichem Umfange abnimmt wie der $\mathrm{Bsb}_{5}$, dass jedoch eine Nitrifizierung des Ammoniaks nicht eintritt. Bei den wenigen Versuchen mit schwachbelasteten Anlagen konnte dagegen mit abnehmender hydraulischer Raumbelastung und abnehmender Belastung mit N-Verbindungen eine zunehmende Nitrifizierung beobachtet werden.

Beim künstlich belüfteten Tropfkörper gestalten sich die Verhältnisse insofern anders, als nach unseren Beobachtungen bei denjenigen Raumbelastungen, die noch einen guten $\mathrm{Bsb}_{5}$-Abbau ergeben, stets eine beträchtliche Nitrifizierung eintritt, wobei aber im allgemeinen der Abbau des organischen Stickstoffs in geringerem Umfange erfolgt als der $B_{s} b_{5}$-Abbau. Der Umsatz der $\mathrm{N}$-Verbindungen gestattet übrigens einen gewissen Einblick in die Arbeitsweise des Tropf körpers. So kann der hohe Nitratgehalt bei gleichzeitigen Anwesenheit verhältnismässig grosser Mengen Ammoniaks und organischen Stickstoffs im Abfluss nur bei Annahme von Kurzschlüssen verstanden werden, worauf schon VIEHL (I94I) hingewiesen hat. Auf Grund der Beziehungen in Abbildung I9 schliessen wir, dass auch bei relativ geringen Raumbelastungen bereits Kurzschlüsse, sogar in einem hochgeschichteten Körper, auftreten können. Einen direkten Beweis für diese Schlussfolgerung liefern die Versuche von DeKEMa und Murray (I942) bzw. DeKema und Krige (1949), die zeigen konnten, dass mittels Hintereinanderschaltung von zwei hohen, künstlich belüfteten Tropf körpern ein bedeutend besserer Wirkungsgrad erzielt werden kann als mit einem einzigen Filterbeet, gleiche Raumbelastungen der Systeme vorausgesetzt. Bei gleichem Rohwasser und vergleichbarer Qualität des Abflusses finden sie, dass bei sogenannter Doppelfiltration die Raumbelastung $\left(R_{b}\right)$ etwa $40 \%$ höher gewählt werden darf. Wie dies bereits früher ausgesprochen 
wurde, besteht deshalb nach unserer Auffassung der abnehmende Reinigungsgrad (Ưbergang von Vollreinigung zu Teilreinigung) bei steigender Raumbelastung eines Tropfkörpers zur Hauptsache einfach in einer Zunahme des Anteils unveränderten Rohwassers im Anlagenabfluss. Es scheint uns deshalb gärungsbiologisch prinzipiell unrichtig, bei Tropfkörpern mit einmaligem Abwasserdurchgang und hohen Belastungen von Teilreinigung zu sprechen.

Wir haben mittels statistischer Auswertung sämtlicher Einzelmessungen aus unseren Experimenten versucht, eine stetige Funktion zwischen der
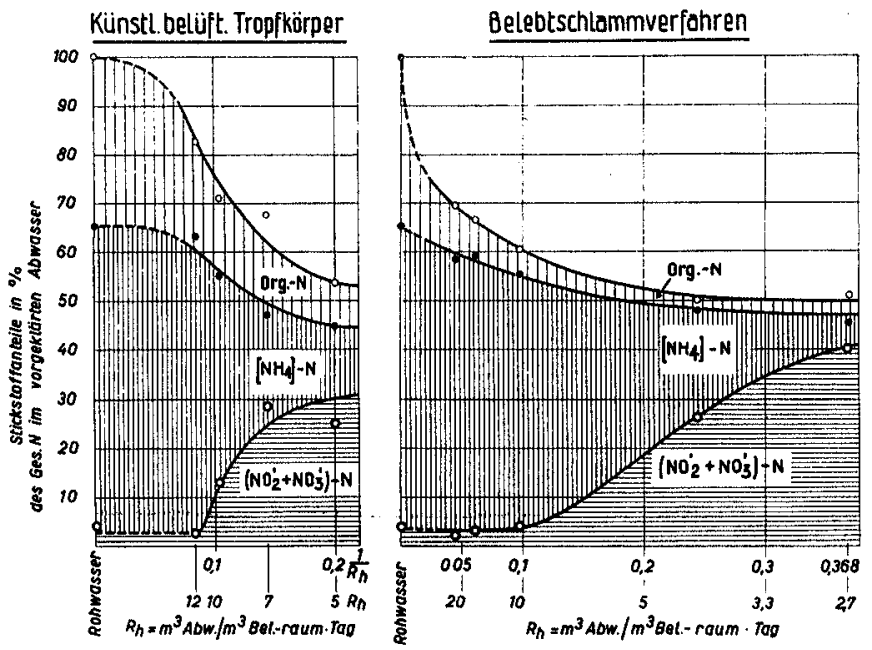

Abb. 18. Prozentuale Veränderung des Gesamtstickstoffgehaltes und der Stickstoffanteile (organischer, Ammonium- und Nitrat- und Nitritstickstoff) bei biologischer Abwasserreinigung mittels: a) künstlich belüftetem Tropfkörper, b) Belebtschlammverfahren.

Ordinate: Prozentuale Stickstoffanteile, bezogen auf den Gesantstickstoff im Rohwasser ( $=100 \%$ ). Abszissen: Reziproke und absolute Werte der hydranlischen Raumbelastung.

Raumbelastung und der Umwandlung der Stickstoffverbindungen bei den beiden Reinigungsverfahren aufzufinden. Die Vereinheitlichung des Materials wurde dadurch erreicht, dass der Anteil der einzelnen N-Komponenten in den Anlageabflüssen in Prozent des Gesamtstickstoffs im jeweiligen Rohwasser berechnet wurde. Die einzelnen Versuche werden wiederum in Gruppen mit ähnlicher Raumbelastung zusammengefasst. Die Mittelung der berechneten prozentualen Anteile der N-Komponenten am Gesamtstickstoff führt in Funktion der reziproken Werte der hydraulischen Raumbelastungen zu den Beziehungen von Abbildung I8a und 
I8b. Die Kurven dieser Darstellungen sind natürlich mit grossen Streuungen behaftet. Sie bringen aber die Beobachtungen anlässlich der Experimente treffend zum Ausdruck. Vor allen Dingen fällt der grosse Unterschied im Abbau des organischen Stickstoffes beim Tropfkörper- und Belebtschlammverfahren ins Auge. Ferner zeigt sich in Übereinstimmung mit den eingangs erwähnten Beobachtungen, dass beim Tropfkörper die Nitrifizierung mit abnehmender Raumbelastung viel rascher zunimmt als bei den Belebtschlammanlagen, ohne dass aber eine entsprechende Reinigung des Abwassers bezüglich des organischen Stickstoffs zu beobachten wäre. Es ist auch auffallend, dass bei der kleinsten von uns beobachteten Belastung des Tropfkörpers $\left(R_{h}=5 \mathrm{~m}^{3} / \mathrm{m}^{3} \cdot \mathrm{Tag}\right)$ der Abbau des organischen Stickstoffs in bedeutend geringerem Umfange erfolgt als beim analog belasteten Belebtschlammverfahren, denn im Tropfkörperabfluss sind noch 10\% des ursprünglichen Gesamtstickstoffs in organischer Form vorhanden, beim Belebtschlammverfahren nur noch rund $3 \%$.

\section{ELIMINATION DES STICKSTOFFS}

Grundsätzlich sind zwei Wege denkbar, um Stickstoffverbindungen aus dem Abwasser mittels biologischer Stoffwechselvorgänge zu entfernen:

I. Fixierung des Stickstoffs in Organismensubstanz und Abtrennung derselben aus dem Abwasser («Inkarnation» des N) als Schlamm.

2. Überführung der Stickstoffverbindungen in elementaren Stickstoff mittels biologischer Denitrifikation.

Die erste Reaktion setzt voraus, dass den Organismen, die Stickstoffverbindungen resorbieren und in Körpersubstanz festlegen sollen, gleichzeitig der nötige Kohlenstoff für den Körperaufbau zur Verfügung steht. Es ist bekannt, dass Mikroorganismen zwischen 8 und $12 \%$ Stickstoff und rund $40 \%$ Kohlenstoff enthalten. Das heisst, dass für jedes Mol N, das festgelegt wird, 3-5 Mol C gebunden werden müssen. Es ist ferner zu veranschlagen, dass für I $\mathrm{g}$ C, das in Organismensubstanz fixiert ist, ein Vielfaches davon für den Energiestoffwechsel der wachsenden Lebewesen benötigt wird. Dieser letztere Kohlenstoff geht in Form von Kohlensäure und anderen Dissimilationsprodukten wieder verloren und fällt für die Kohlenstoff-Stickstoff-Bilanz aus. Auf Grund der Analysen in Serie X, wo wir neben dem $N$ auch den Gesamtgehalt an organischem $C$ im $A b-$ wasser untersucht haben, ist ersichtlich, dass im normalen städtișchen Abwasser ein C-N-Verhältnis von rund 2: I bis 3:I (Tabelle 3 ) besteht. 
Es ist also ein sehr grosses Defizit an assimilierbaren C-Verbindungen vorhanden, so dass es nicht erstaunen muss, wenn der N-Elimination mittels Bindung in Organismenschlamm enge Grenzen gesetzt sind. Anderseits kann natürlich durch künstlichen Zusatz vergärbarer C-Verbindungen ins Abwasser das C-N-Verhältnis günstiger gestaltet werden, was sofort $\mathrm{zu}$ entsprechend höheren N-Eliminationen führt (vgl. Hinweis von SAwYER, I944).

Die Denitrifikation von Ammonium- und eventuell andern N-Verbindungen auf bakteriellem Wege ist in Abwasserreinigungsanlagen wohlbekannt und wurde im Zusammenhang mit dem Problem der Blähschlammbildung in Belebtschlammanlagen mehrfach beschrieben. Hingegen ist ihr Umfang beim normalen Gang der Abwasserreinigung mittels Belebtschlamm- oder Tropf körperverfahren noch ganz unklar.

Aus unseren Versuchen geht zunächst hervor, dass bei beiden geprüften Systemen mehr oder weniger grosse Mengen Stickstoff aus dem Abwasser verschwinden. Die prozentuale Elimination (bezogen auf die Gesamtstickstoffmenge im Rohwasser) ist für die Versuchsgruppen mit ähnlicher Raumbelastung aus den Abbildungen $18 a, b$, ersichtlich (oberste Kurven). Dieselbe beträgt beim Belehtschlammverfahren bei Raumbelastungen von rund $22 \mathrm{~m}^{3} / \mathrm{m}^{3}$. Tag etwa $33 \%$ des im Rohwasser zugeführten Stickstoffs. Mit abnehmender Raumbelastung nimmt die N-Elimination zu und nähert sich in unsern Versuchen bei geringen Raumbelastungen einem Grenzwert von zirka 50\%. Beim künstlich beliufteten Tropfkörper erreicht die Elimination bei Raumbelastungen von $\sim 5 \mathrm{~m}^{3} / \mathrm{m}^{3}$. Tag gleichartige Werte wie beim Belebtschlammverfahren, nimmt aber mit steigender Raumbelastung rasch ab.

In ausländischen Anlagen, von denen wir vor allem die Messungen von MOHLMANN an den schwachbelasteten Belebtschlammanlagen in Chicago zitieren wollen, ergeben sich N-Eliminationen von 30 bis maximal $45 \%$ (Tabelle 22). Wir haben deshalb den Eindruck, dass unsere hochbelasteten Belebtschlammanlagen relativ hohe Eliminationsleistungen zeigen (Tabelle 12).

Vergleichsangaben für den künstlich belïfteten Tropfkörper stehen uns von Anlagen aus der Praxis nicht zur Verfügung. Hingegen ist es interessant, die Angaben von LANZ (I937) beizuziehen, die seinerzeit an einem kleinen Versuchstropfkörper der Stadt Zürich im Werdhölzli gewonnen wurden. LANZ fand N-Eliminationen von höchstens $25 \%$ bei einer Raumbelastung von $4 \mathrm{~m}^{3} / \mathrm{m}^{3}$. Tag und stellte ebenfalls den raschen Rückgang der Eliminationsleistung mit zunehmender Raumbelastung fest (Tabelle 23). 
Tabelle 22

Stickstoffelimination bei amerikanischen Belebtschlammanlagen

\begin{tabular}{|c|c|c|c|c|c|c|}
\hline \multirow[b]{2}{*}{ Anlage } & \multirow[b]{2}{*}{$R_{h}$} & \multirow{2}{*}{$\begin{array}{c}\text { Gesamt-N } \\
\text { Zufl uss } \\
\text { (vor- } \\
\text { geklärt) } \\
\text { mg N/l }\end{array}$} & \multirow{2}{*}{$\begin{array}{l}\text { Gesamt-N } \\
\text { Abfluss } \\
\text { (nach- } \\
\text { geklärt) } \\
\text { mg N/I }\end{array}$} & \multicolumn{2}{|c|}{$\mathrm{N}$-Elimination } & \multirow[b]{2}{*}{ Quelle } \\
\hline & & & & $\operatorname{mg~N} / 1$ & $\begin{array}{c}\% \text { bez. } \\
\text { Roh- } \\
\text { wasser }\end{array}$ & \\
\hline $\begin{array}{l}\text { Chicago SW, } 1946 \\
\text { Chicago }\end{array}$ & 2,62 & 20,7 & 11,2 & 9,5 & 46 & Jahresbericht 1946 \\
\hline Calumet, 1946 & 3,7 & 19,9 & 14,2 & 5,7 & 28,6 & Jahresbericht 1946 \\
\hline Chicago N, 1946 & 3,65 & 19,1 & 10,6 & 8,5 & 44,5 & Jahresbericht 1946 \\
\hline Jackson, Mich., 1946 & 3,4 & 21,1 & 15,8 & 5,3 & 25 & Jahresbericht 1946 \\
\hline
\end{tabular}

Tabelle 23

Stickstoffelimination bei Tropfkörpern

\begin{tabular}{|l|r|c|c|c|c|c|}
\hline \multicolumn{1}{|c|}{ Anlage } & $R_{h}$ & $\begin{array}{c}\text { Gesamt-N } \\
\text { Zufluss } \\
\text { (vor- } \\
\text { geklärt) } \\
\text { mg N/1 }\end{array}$ & $\begin{array}{c}\text { Gesamt-N } \\
\text { Abfluss } \\
\text { (nach- } \\
\text { geklärt) } \\
\text { mg N/1 }\end{array}$ & mg N/1 & $\begin{array}{c}\text { N-Elimination } \\
\text { Roh- } \\
\text { wasser }\end{array}$ & Quelle \\
\hline Versuchstropflkörper & 4 & 33,3 & 25,1 & 8,2 & 24,6 & \\
(künstlich belüftet) & 6 & 34,6 & 28,2 & 6,4 & $\mathbf{1 8 , 5}$ & Dissertation \\
der Stadt Zürich & 8 & 25,8 & 23,0 & 2,8 & $\mathbf{1 1 , 3}$ & LANZ (1938) \\
1935/36 & 10 & 22,6 & 18,5 & 4,1 & 22,2 & \\
Aurora, Ill., 1946 & & 24,0 & 16,7 & 7,3 & 30,4 & S.W. J. 1947 \\
(offene Tropfkörper) & $?$ & 24,0 & 16,0 & \\
\hline
\end{tabular}

\section{StickstoffBilanZ DER REINIGUNGSANLAGEN}

ZEHENDER hat bereits 1949 darauf hingewiesen, dass ein Einblick in den N-Umsatz von Reinigungsanlagen nur an Hand von vollständigen Stickstoff bilanzen gewonnen werden kann und belegte an Beispielen aus früheren Versuchen auf unsern Anlagen, in welchem Umfange der Stickstoff beispielsweise mit dem Überschußschlamm eliminiert wird. In Tabelle 24 geben wir eine gesamthafte Zusammenstellung von N-Bilanzen 
derjenigen Experimente wieder, für die unseres Erachtens genügend genaue Unterlagen bestehen. Insbesondere verweisen wir auf die letzte Kolonne dieser Tabelle, in welcher die entstehenden Stickstoffdefizite berechnet sind, wenn man den Gesamtzufluss dieses Elementes in eine biologische Anlage und den Gesamtabfluss ( $\mathrm{N}$ im Anlagenabfluss $+\mathrm{N}$ im abgelassenen Überschußschlamm) einander gegenüberstellt. Wegen der aus verschiedenen Gründen ziemlich grossen Schwankungen in den täglichen Überschußschlammengen können diese Berechnungen nur an Hand von Abschnittsmittelwerten vorgenommen werden.

Es zeigte sich, dass beim Belebtschlammperfabren regelmässige N-Defizite in der Grössenordnung von 20 bis $30 \%$ auftreten. Ferner ist ersichtlich, dass diese Defizite einen Zusammenhang mit dem Umfang der Sauerstoffversorgung im Belüftungsraum der Anlagen aufzuweisen scheinen. Obschon diese N-Bilanzen nicht sehr genau sein können, müssen wir die Defizite als reell betrachten, da die Fehler der Messgrössen, die den Berechnungen zugrunde liegen, zufällig verteilt sind, die Defizite dagegen systematisch auftreten.

Wir sind nicht in der Lage, auf Grund entsprechender Versuche heute schon die Ursache der N-Defizite erklären zu können. Der Schluss liegt jedoch nahe, dass sie auf Grund von Denitrifikationsvorgängen bei der Abwasserreinigung zustande kommen. Es ist weiteren Untersuchungen vorbehalten, Klarheit in diese Fragen zu bringen. Es ist denkbar, eventuell auf diesem Wege die N-Elimination zu erhöhen, die ja gemäss unseren vorhergehenden Ausführungen mittels Schlammbildung bei städtischem Abwasser gewisse Grenzen nicht übersteigen kann.

Auch beim Tropfkörper treten hohe Defizite auf, wobei eine deutliche Abhängigkeit zwischen der Raumbelastung und der Grösse der N-Verluste zu bestehen scheint. Da mit abnehmender Raumbelastung die Nitrifizierung im Tropf körper zunimmt, könnte man auch einen Zusammenhang zwischen Nitrifizierung und Denitrifizierung vermuten. Eine genauere Aussage ist zur Zeit leider nicht möglich, da die Defizitwerte bei den Tropfkörperversuchen sehr viel ungenauer sind als bei den Belebtschlammversuchen, denn die genaue Schlammproduktion einer Tropfkörperanlage ist innerhalb relativ kurzer Beobachtungsperioden wegen der Retention schwierig zu erfassen. Aus diesen Gründen müssen wir darauf verzichten, weitergehende Schlüsse aus unseren Beobachtungen zu ziehen: Wir glauben aber, dass auf Grund unserer Experimente beim Tropfkörper die eventuelle Denitrifikation nicht höher veranschlagt werden darf als beim Belebtschlammverfahren. 
Tabelle 24

Stickstoff bilanzen der Reinigungsanlagen in einzelnen Versucbsabschnitten

(Eingeklammerte Ziffern: Wegen Schlammretentionen innerhalb der Analysenperiode sind diese Defizitwerte wahrscheinlich zu hoch)

\begin{tabular}{|c|c|c|c|c|c|c|}
\hline \multirow[b]{2}{*}{ Versuch } & \multirow{2}{*}{$\begin{array}{c}\begin{array}{c}\text { N-Zufluss } \\
\text { (Rohwasser) }\end{array} \\
\text { kg N/Tag }\end{array}$} & \multicolumn{3}{|c|}{$\mathrm{N}$-Abfluss } & \multicolumn{2}{|c|}{ Bilanzwert } \\
\hline & & $\begin{array}{c}\left.\mathrm{NB}^{1}\right) \\
\text { Abfluss } \\
\mathrm{kg} \text { N/Tag }\end{array}$ & $\begin{array}{l}\text { Überschuß- } \\
\text { schlamm } \\
\text { kg N/Tag }\end{array}$ & $\begin{array}{c}\text { Total } \\
\text { kg N/Tag }\end{array}$ & $\mathrm{kg} \mathrm{N} / \mathrm{Tag}$ & $\begin{array}{l}\text { Prozent } \\
\text { des } \\
\text { Zuflusses }\end{array}$ \\
\hline \multicolumn{7}{|c|}{ I. Belebtschlammversuche } \\
\hline $\begin{array}{ll}\text { IX } & a_{1} \\
\text { IX } & a_{2} \\
\text { IX } & o \\
\text { X } & o_{1} \\
\text { X. } & o_{1} \\
\text { X } & a_{1} \\
\text { X } & a_{2} \\
\text { X } & o_{2} \\
\text { X } & a_{3} \\
\text { XI } & o_{1} \\
\text { XI } & o_{2}\end{array}$ & $\begin{array}{l}20,39 \\
28,67 \\
12,25 \\
26,52 \\
24,80 \\
26,51 \\
24,80 \\
19,96 \\
19,96 \\
27,99 \\
25,57\end{array}$ & $\begin{array}{r}11,14 \\
16,57 \\
6,99 \\
17,31 \\
15,13 \\
16,69 \\
14,58 \\
14,55 \\
14,80 \\
17,97 \\
16,93\end{array}$ & $\begin{array}{l}3,87 \\
5,39 \\
2,21 \\
4,46 \\
4,76 \\
4,69 \\
5,36 \\
5,21 \\
4,79 \\
5,43 \\
7,53\end{array}$ & $\begin{array}{r}15,01 \\
21,96 \\
9,20 \\
21,77 \\
19,89 \\
21,38 \\
19,94 \\
19,76 \\
19,59 \\
23,40 \\
24,46\end{array}$ & $\begin{array}{l}-5,38 \\
-6,71 \\
-3,05 \\
-4,75 \\
-4,91 \\
-5,14 \\
-4,86 \\
-0,20 \\
-0,37 \\
-4,59 \\
-1,11\end{array}$ & $\begin{array}{l}-26 \\
-23 \\
-25 \\
-18 \\
-20 \\
-19 \\
-20 \\
-1 \\
-2 \\
-16 \\
-4\end{array}$ \\
\hline \multicolumn{7}{|c|}{ II. Tropfkörperversuche } \\
\hline $\begin{array}{ll}\text { VIII } & 2 \\
\text { VIII } & 4 \\
\text { VIII } & 6 \\
\text { IX } & \\
\text { IX } & 2 \\
\text { X } & \end{array}$ & $\begin{array}{c}18,5 \\
26,6 \\
19,92 \\
9,4 \\
19,0 \\
24,7\end{array}$ & $\begin{array}{l}12,3 \\
18,3 \\
13,85 \\
5,1 \\
14,3 \\
19,6\end{array}$ & $\begin{array}{c}0,55 \\
1,71 \\
1,49 \\
(0,8) \\
(0,5) \\
(1,0) \\
\end{array}$ & $\begin{array}{l}12,85 \\
20,11 \\
15,34 \\
(5,9) \\
(14,8) \\
(20,6)\end{array}$ & $\begin{array}{l}-5,65 \\
-6,49 \\
-4,58 \\
(-3,5) \\
(-4,2) \\
(-4,1)\end{array}$ & $\begin{array}{l}-30 \\
-25 \\
-23 \\
(-37) \\
(-22) \\
(-16,5) \\
\end{array}$ \\
\hline
\end{tabular}

\section{Phosphorelimination}

Leider wurde die Untersuchung der Phosphorbilanz bei unsern Experimenten erst relativ spät in regelmässiger Weise aufgenommen, so dass uns über die Phosphorelimination heute weniger Unterlagen zur Verfügung stehen als beim Stickstoff.

Zunächst ist festzustellen, dass der Phosphor mengenmässig bei den biologischen Umsetzungen in einer Reinigungsanlage nur in geringem Umfange beteiligt ist, da der P-Gehalt und der P-Bedarf der Flora in den Reinigungskörpern klein ist.

Aus den Untersuchungen der letzten Jahre zeigt sich, dass das abgesetzte Rohwasser von Zürich bei normaler Konzentration im Durchschnitt rund 
2-3 mg P/l enthält; davon sind rund $50 \%$ in den Schwebestoffen gebunden. Aus Tabelle II geht hervor, dass bei der Reinigung mit dem Belebtscblammverfabren zwischen 30 und $50 \%$ des Gesamtphosphors aus dem Rohwasser verschwindet. Eine Abhängigkeit der P-Elimination von der Raumbelastung ist nicht nachzuweisen.

Beim Tropfkörperverfabren stehen uns nur Analysenergebnisse aus Versuchen mit hohen Raumbelastungen (Serien $\mathrm{IX}_{\mathbf{2}}$ und X) zur Verfügung, in welchen 25 bzw. 18\% P-Elimination gemessen wurden. Es ist anzunehmen, dass bei niedrigeren Tropfkörperbelastungen die P-Elimination eher geringer ausfallen muss, da dann die vermehrt einsetzende Selbstverzehrung des Schlammes den aus dem Abwasser aufgenommenen Phosphor teilweise wieder freisetzt.

Anlässlich einer speziellen Untersuchung, bei der beim Belebtschlammverfahren Tagesmittelproben des Rohwassers und des Anlagenabflusses vor der Analyse mittels Sterilfiltration (durch Membranfilter) von sämtlichen Schwebestoffen befreit wurden, versuchten wir einen ersten Einblick in den Phosphorumsatz bei der Abwasserreinigung zu gewinnen. Die Anlage arbeitete damals mit einer Raumbelastung von $22 \mathrm{~m}^{3} / \mathrm{m}^{3} \cdot \mathrm{Tag}$ (November 1950). In Tabelle 25 sind die Phosphormittelwerte aus drei innerhalb von to Tagen gefassten Proben enthalten. Gleichzeitig haben wir die aus den Differenzen der Trockenrückstände in den filtrierten und unfiltrierten Abwasserproben sich ergebenden Schwebestoffgehalte in die Tabelle eingetragen. Es ergibt sich aus dieser Untersuchung, dass die Phosphorelimination, gemessen an den unfiltrierten Proben, 37;7\% betrug, währenddem durch die Filtration des Rohwassers $54,5 \%$ Phosphor zurückgehalten wird. Die P-Elimination war also geringer, als dies dem Phosphorgehalt in den Schwebestoffen des Rohwassers entsprach. Anderseits lässt sich berechnen, dass der P-Gehalt der Schwebestoffe des Rohwassers lediglich $0,8 \%$, dagegen derjenige des Belebtschlammes (gleichbedeutend mit den Schwebestoffen des Anlageabflusses) 2,3\% beträgt. Es erfolgt somit ein weitgehender Umbau der P-Verbindungen im Laufe der Abmasserreinigung, wobei aber ein Teil des in Schwebestoffen des Rohwassers gebundenen Phosphors in Lösung geht. Wir schliessen aus unseren Beobachtungen, dass eine Erhöhung der P-Elimination bei der Abwasserreinigung nur möglich erscheint, wenn noch wesentlich mehr Schlammtrockenstoffe aus den Anlagen abgeschieden werden können, als dies heute der Fall ist. Diese Bedingung ist aber ähnlich wie bei der Stickstoffelimination an den Gehalt assimilierbaren Kohlenstoffes im Rohwasser gebunden. Über die Phosphorelimination in Tropfkörper- und Belebt schlammanlagen geben 
Tabelle 25

Phosphorgehalt des gereinigten und ungereinigten Abwassers somie der Scbobebestoffe

\begin{tabular}{|c|c|c|}
\hline & Rohwasser & $\begin{array}{c}\text { Abfluss des } \\
\text { Nachllärbeckens }\end{array}$ \\
\hline $\begin{array}{l}\text { Schwebestoffe . . . . . . . . } \mathrm{mg} / \mathrm{l} \\
\text { Phosphor } \\
\text { vor Filtration. . . . . . . . . } \mathrm{mg} / \mathrm{l} \\
\text { nach Filtration . . . . . . } \mathrm{mg} / \mathrm{l} \\
\text { in Schwebestoffen . . . . . . . . } \\
\text { Phosphorelimination } \\
\text { durch Filtration allein. . . . . . . . } \\
\text { durch biologische Reinigung (bez. auf } \\
\text { unfiltrierte Proben) . . . . . . . . }\end{array}$ & $\begin{array}{c}133 \\
1,99 \\
0,91 \\
0,9 \% \\
54,5 \%\end{array}$ & $\begin{array}{l}36 \\
1,21 \\
0,68 \\
2,3 \% \\
\\
\\
37,7 \%\end{array}$ \\
\hline
\end{tabular}

Jenkins und Lockett (I943) Prozentangaben, die mit unseren Beobachtungen weitgehend iibereinstimmen. Sie schätzen, dass mittels Abwasserreinigung $46-60 \%$ des vom Abwasser transportierten Phosphors aus dem Schlamm zurückgewonnen werden könnten (Vorklärung inbegriffen).

\section{E. Schlammproduktion und Schlammzusammensetzung}

\section{I. ÜBERSCHUSSSCHLAMMENGEN}

Die in den Tabellen I 3 und I7 zusammengestellten, mittleren absoluten und spezifischen Mengen produzierten Überschußschlammes beim Tropfkörper- bzw. Belebtschlammverfahren lassen keine gesetzmässige Abhängigkeit von den Betriebsdaten der Anlagen in den einzelnen Versuchsabschnitten erkennen. Man findet ziemlich gleichförmig, dass beim Belebtschlammeerfabren rund $80-120 \mathrm{~g}$ Trockensubstanz pro Kubikmeter Abwasser anfallen. Bei den Tropfkörperversuchen sind diese Ziffern zwei- bis dreimal geringer, wie dies nicht anders zu erwarten ist, da ja der Schlamm im Tropfkörper selbst weitgehend abgebaut wird. Leider stehen uns speziell für die höheren Tropf körperbelastungen keine zuverlässigen Zahlen zur Verfügung, da in jenen Experimenten starke Schlammretentionen innerhalb der Analysenperioden stattfanden.

Betrachtet man statt der Gesamtmenge produzierter Schlammtrockensubstanz nur den Anteil der organischen Komponente (Glühverlust), so lässt sich beim Belebtschlammverfabren immerhin feststellen, dass ein gewisser Zusammenhang zwischen der Produktion organischer Substanz 
und der Raumbelastung mit $\mathrm{Bsb}_{5}\left(R_{b}\right)$ besteht. In Abbildung I9 haben wir die pro Kubikmeter Belüftungsraum anfallende organische Substanz im Überschußschlamm in Funktion der Raumbelastung $R_{b}$ eingetragen. Man kann durch das Feld der Einzelpunkte im Sinne einer allgemeinen Richtlinie eine Gerade legen, die zum Ausdruck bringt, dass in erster Annäherung der organische Anteil des Úberschußschlamms proportional

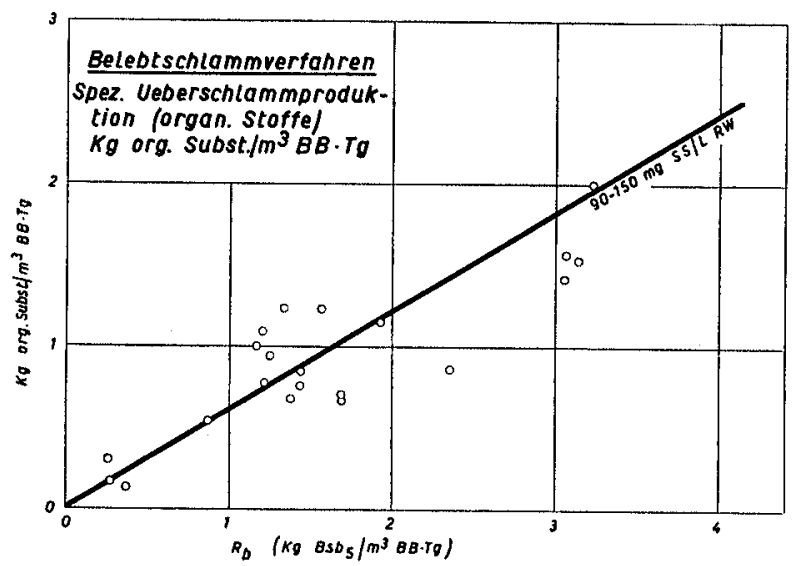

Abb. 19. Schlammprodulition in Funktion der Raumbelastung mit $\mathrm{Bsb}_{5}$ beim Belebtschlammverfahren.

Ordinate: Organische Substanz in der täglichen Uberschußschlammenge (kg organische Sub$\operatorname{stanz} / \mathrm{m}^{3}$ Belüftungsraum $\left.\cdot \mathrm{Tag}\right)$. Abszisse: $R_{b}\left(\mathrm{~kg} \mathrm{Bsb} / \mathrm{m}^{3} \cdot \mathrm{Tag}\right)$.

$\mathrm{zu} R_{b}$ ist. Als Proportionalitätsfaktor kann ein Wert von $\sim 0,6$ angesetzt werden. Es ist in Erinnerung zu rufen, dass diese Ziffer gültig ist für Rohwasser mit einem Schwebestoffgehalt von 90-I50 mg/l.

Die grosse Streuung der Werte für die Überschußschlammproduktion ist an sich nicht erstaunlich, da die letztere von einer grossen Zahl von Variablen abhängig ist. HEUKELEKIAN (I95I) hat versucht, auf Grund von Laboratoriumsexperimenten eine gesetzmässige Abhängigkeit zwischen der Überschußschlammproduktion, der Raumbelastung mit $\mathrm{Bsb}_{5}$ und der Konzentration des Belebtschlammes im Belüftungsbecken aufzustellen. Eine entsprechende Auswertung unserer Messungen zeigte, dass diese Beziehung für unsere Versuche in keiner Weise gilt.

In Anlehnung an die Beobachtung bei Tropfkörpern könnte man vermuten, dass ein wesentlicher Faktor, der die Menge produzierter organischer Substanz bestimmt, die Zeit ist, während welcher der Belebtschlamm 
in der Anlage verweilt. Tatsächlich kann man in Laboratoriumsversuchen feststellen, dass bei fortgesetzter Belüftung von Belebtschlamm eine äusserst intensive Selbstverzehrung stattfindet. GouLd (1942) hat als Mass für die Aufenthaltszeit eines Belebtschlammes in einer Anlage den Begriff des sogenannten Schlammalters eingeführt, den er definiert als Verhältnis der Gewichte der Schwebestoffe im Belüftungsraum $S_{B}\left(\mathrm{~kg} / \mathrm{m}^{3}\right)$ und der

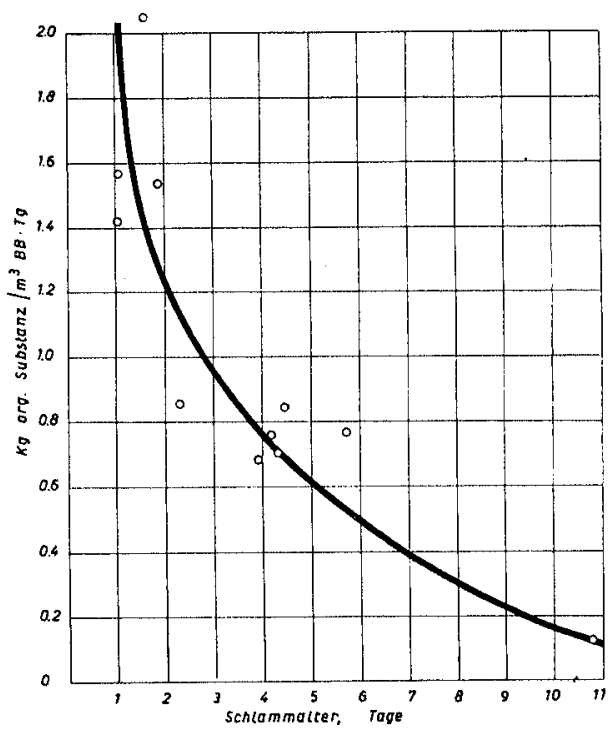

Abb. 20. Schlammproduktion in Funktion des Schlammalters beim Belebtschlammverfahren. Ordinate: Organische Substanz in der täglichen Überschußschlammenge (kg organische Substanz $\left./ \mathrm{m}^{3} \cdot \mathrm{Tag}\right)$. Abszisse: Schlammalter in Tagen.

täglich mit dem Rohwasser zugeführten Schwebestoffe $S_{R}\left(\mathrm{~kg}^{3} / \mathrm{m}^{3} \cdot \mathrm{Tag}\right)$. Unter Einführung des Schlammalters lässt sich in grober Annäherung feststellen, dass die vermutete Selbstverzehrung von Schlamm beim Belebtschlammverfahren ähnlich wie beim Tropfkörper eintritt und dann dic Schlammproduktion abnimmt, sobald das Schlammalter ansteigt (Abb.20). Wie bei unserer ersten Beziehung (Abb. 19) handelt es sich aber auch hier nur um einen approximativen Zusammenhang.

Von besonderer Wichtigkeit für die Praxis sind die Ziffern für das spezifische Volumen des gebildeten Überschußschlammes. Unsere Untersuchungen bestätigen in dieser Hinsicht die allgemein bekannte Grund- 
zahl, dass pro Kubikmeter Rohwasser beim Belebtscblammverfabren mit $\mathrm{I} 2$ bis I6 $1 \mathrm{Naßschlamm} \mathrm{zu}$ rechnen ist. Im Hinblick auf die Weiterbehandlung des Überschußschlamms ist deshalb die nachträgliche Eindickung in speziellen Installationen unumgänglich, wobei wir in Anbetracht der grossen O-Zehrung des Schlammes und aus andern Gründen zur Auffassung kommen, dass eine Rückleitung des Überscbußschlamms in die Vorklärbecken nicht vorteilhaft ist, sondern spezielle Schlammeindickungsbecken vorzuziehen sind.

Beim Tropfkörper ergeben sich spezifische Schlammengen von 2-3 I/ $\mathrm{m}^{3}$ Rohwasser in denjenigen Versuchsabschnitten, bei denen die Entschlammung des Filterbeetes als normal beurteilt werden konnte. Diese Ziffer stimmt mit den konventionellen Angaben für hochbelastete Tropfkörper überein (IMHOFF, Taschenbuch der Stadtentwässerung [1951]: 2,7 1/m³. Tag).

\section{SchlammzusammensetzunG}

In den Serien IX, X und XI der Belebtscblammversuche wurde die $\mathrm{Zu}-$ sammensetzung des Überschußschlammes genauer untersucht. Bezüglich der Ergebnisse verweisen wir auf Tabelle $\mathrm{I} 3$ und möchten nur auf folgende interessante Punkte hinweisen:

Die beträchtlichen Schwankungen im Aschengebalt des Schlammes zwischen einem Minimum von $25 \%$ und einem Maximum von $42 \%$ konnten wir bis jetzt nicht in irgendeinen Zusammenhang mit der Betriebsweise der Anlagen stellen. Wohl weisen die Schlämme der Vollreinigungsanlagen im allgemeinen höhere Aschengehalte auf, was mit einer gewissen Selbstverzehrung bei den niederen Raumbelastungen in Zusammenhang gebracht werden könnte. Hierfür sprechen auch die prozentualen Gehalte an organischem Kohlenstoff. Es bestehen aber auch zahlreiche Ausnahmen von dieser Regel, und wir müssen feststellen, dass wir noch nicht in der Lage sind, die Faktoren genauer zu umschreiben, welche bestimmte Schlammzusammensetzungen bedingen. Insbesondere gilt dies auch für die Stịckstoff-, Phosphor- und Kaliumgehalte, die im Hinblick auf die Schlammverwertung (zum Beispiel als Dünger) von besonderem Interesse sind. Immerhin ist bemerkenswert, dass der Belebtschlamm in zahlreichen Experimenten im Mittel bis zu über 6,5\% Stickstoff enthielt, was dem Gehalt eines hochwertigen organischen Düngemittels entspricht. Insbesondere ist auch eine Gegenüberstellung der Belebtschlammzusammensetzung mit dem Vorklärschlamm bzw. der Schwebestoffe im abgesetzten Rohwasser beachtenswert, die zeigt, dass der Belebtscblamm grundsätzlich 
pon den Feststoffen perschieden ist, die vom roben Abmasser transportiert werden, oder mit andern Worten, dass ein weitgehender Stoffumbau im Laufe der Abwasserreinigung stattfindet. Der deutlichste Hinweis dafür ergibt sich wohl aus den Ziffern für den Aminosäurestickstoff in den Schlammhydrolysaten (Belebtschlamm 2-4\%, Rohschlamm I,I-I,4\%).

In Serie $\mathrm{Xa}_{2}^{20}$ machte sich der Zusatz von $20 \mathrm{mg} / \mathrm{l}$ Asbest zum Belebtschlammverfahren in einer Erhöhung des Aschegehaltes bzw. Erniedrigung der organischen Schlammkomponenten deutlich bemerkbar, wenn man zum Vergleich die Zusammensetzung der Schlämme in den Parallelversuchen heranzieht. Der früher übliche Asbestzusatz von I mg/l verursachte jedoch keine genauer erkennbare Veränderung des Schlammaufbaues.

Naturgemäss ist die Zusammensetzung des Überschußschlammes aus dem künstlich belïfteten Tropfkörper vom Belebtschlamm verschieden, da er ja innerhalb des Filters bereits erheblichen Umsetzungen unterworfen ist. Gesamthaft betrachtet, stellte sich in allen Versuchen der N-Gehalt niedriger als beim Überschussbelebtschlamm.

Es wäre zweifellos von Interesse, unsere Messungen bezüglich Schlammproduktion und Schlammzusammensetzung noch nach weiteren betriebstechnischen und biochemischen Gesichtspunkten auszuwerten, doch scheint uns dies erst angezeigt zu sein, wenn auf Grund von speziellen, noch anzustellenden Versuchen die Zusammenhänge schärfer herausgeschält sind, die als Leitfaden für detaillierte Auswertungsarbeiten dienen können.

\section{VIERTER TEIL}

\section{SCHLUSSBEMERKUNGEN UND ZUSAMMENFASSUNG}

Wir haben in den vorstehenden Kapiteln die wichtigsten Ergebnisse unserer Versuche unter Weglassung einer grossen Zahl von Einzelheiten zusammengefasst, um vor allem die für die Praxis wichtigen, grundlegenden Resultate besser hervortreten zu lassen. Insbesondere haben wir unsere ausgedehnten Untersuchungen über die biologischen Verhältnisse in den geprüften Reinigungssystemen nicht erwähnt. Es zeigte sich nämlich, dass weder beim Tropfkörper- noch beim Belebtschlammverfahren spezielle, nicht schon bekannte Zusammenhänge zwischen Betriebsweise und Schlammbiozönose gefunden werden konnten. Naturgemäss erwiesen sich unsere hochbelasteten Belebtschlämme bei den stets niedrigen Sauer- 
stoffspannungen in den Belüftungsbecken immer relativ arm an identifizierbaren Organismen (Protozoen). Kleinste Flagellaten, die nicht mehr untersucht wurden, fanden sich dagegen oft in grösserer Zahl. Die Ziliatenfauna nahm mit zunehmender Verbesserung der Sauerstoffverhältnisse im Schlamm stets an Zahl zu, wie dies aus zahlreichen Beobachtungen bekannt ist. Die Bedeutung der Ziliaten für die Klarheit der Anlagenabflüsse, wie sie von BARKER (r949) und mit dem demonstrativen Experiment von SUDGEN und LLOYD (1950) herausgestellt wurde, liess sich in mehreren Versuchen bestätigen. Unsere Schlämme enthielten neben der Hauptmasse von Zoogloen stets eine geringe Menge von Scheidenbakterien (meist Cladothrix dichotoma). Die gleichmässige Zusammensetzung des Abwassers führte jedoch nie zu einer derartigen Zunahme dieser Organismen, dass Schlammindizes entstanden, wie sie bei eigentlichem Blähschlamm zu beobachten sind.

Wie sich im Laufe der Versuche zeigte, ist das Belebtschlammverfahren für die Reinigung von städtischem Abwasser dem Tropfkörpersystem hinsichtlich spezifischem Bauvolumenbedarf der Reinigungskörper stark überlegen. Dies mag erstaunlich sein, wenn man berücksichtigt, dass in einem Tropf körper pro Kubikmeter Füllmaterial nach ToMLINSON (I950) zwischen 4500-10000 g Schlamm vorhanden sind (abhängig von der Belastung), während in einem Belüftungsbecken bisher $3000-3500 \mathrm{~g} / \mathrm{m}^{3}$ Höchstwerte darstellten. Aus diesen Zahlen sollte sich doch eigentlich eine grössere Leistungsfähigkeit der Tropf körper ergeben als beim Belebtschlammsystem, denn die Abbauleistung eines biologischen Verfahrens ist in erster Linie von der Masse der beteiligten Gärungsorganismen abhängig (die Artenzusammensetzung, die ebenfalls wesentlich erscheint, ist weitgehend vom Chemismus des Abwassers sowie von den in der Anlage geschaffenen physikalischen Bedingungen beherrscht und damit nicht ohne weiteres einer Lenkung zugänglich). Wenn sich nun die Abbauleistung einer Belebtschlammanlage dennoch als grösser erweist, so hat man daraus zu schliessen, dass in Tropfkörpern (von Durchflusskurzschlüssen soll abgesehen sein) nur ein verhältnismässig kleiner Teil der Organismenmasse direkt an der Umsetzung der Abwasserstoffe beteiligt ist. In Anbetracht der im Vergleich zu Belebtschlamm geringen Oberflächenentwicklung eines Tropf körperrasens ist dies leicht einzusehen. Ein grosser Anteil des Dissimilationsvermögens der Biomasse eines Tropf körpers wird ferner zum Abbau der zugeführten Schlammstoffe sowie der sekundär gebildeten Organismen verbraucht. Hieraus erklärt sich auch die Tatsache, dass Abwässer, die zu einem Belebtschlamm mit technisch schwierig zu bewälti- 
genden Eigenschaften (hoher Index) führen, von einem Tropfkörper verarbeitet werden können, weil die Organismen der ersten Abbaustufen (die bei gleichem Rohwasser identisch sind mit denjenigen im Belebtschlammsystem) einer weiteren Umsetzung verfallen, bevor sie ins Nachklärbecken gelangen. Es ist deshalb verständlich, dass zur Reinigung von Abwässern, die spezielle gärungsbiologische Voraussetzungen bieten, Tropfkörper beim gegenwärtigen Stand der Erfahrungen günstiger sein können als Belebtschlammanlagen. Auch sind sie für Kleinanlagen (weniger als Iooo angeschlossene Einwohnergleichwerte) wegen ihrer einfachen Betriebsführung zweifellos sehr vorteilhaft. Zur Reinigung der Abwässer grösserer Gemeinden erweist sich aber das Belebtschlammverfahren zur Zeit als das weitaus leistungsfähigste.

\section{ZUSAMMENFASSUNG}

I. In Versuchsanlagen technischer Dimensionen wurde die Abwasserreinigung mit dem Belebtschlammverfahren und mit dem künstlich belüfteten Tropfkörper systematisch untersucht, um vor allem bezüglich der maximalen Leistungsfähigkeit der Systeme Aufschluss zu erhalten. Ferner wurde nach stetigen Beziehungen zwischen Belastung und Qualität des Abflusses gesucht, um Dimensionierungsgrundlagen für hochbelastete Anlagen zu schaffen.

2. Es ist möglich, sowohl für das Belebtschlammverfahren wie für den künstlich belüfteten Tropfkörper innerhalb gewisser Genauigkeitsgrenzen stetige Funktionen zwischen der Raumbelastung mit $\mathrm{Ab}$ wasser bzw. Bsb $b_{5}$ und der Abflussqualität bezüglich Bs $b_{5}$ aufzustellen, die für die Dimensionierung von Reinigungsanlagen gute Unterlagen bieten (Abwasser mit überwiegend städtischem Charakter und heute gebräuchliche technische Einrichtungen vorausgesetzt).

3. Es ist möglich, auch über die Entfernung eutropbierender Abmasserstoffe (Phosphor, Stickstoff) ziemlich gute Voraussagen für die beiden Reinigungssysteme bei verschiedenen Raumbelastungen zu machen.

4. Die vielen Versuche gestatten die Angabe gutbelegter Mittelwerte über die spezifische Überschußschlammproduktion der beiden Verfahren. Für Belebtschlammanlagen konnte eine approximative Funktion für die spezifische Überschußschlammenge (bezogen auf Belüftungsraum) angegeben werden, unter Voraussetzung einer bekannten Raumbelastung mit $\mathrm{Bsb}_{5}$ und einer Schwebestoffkonzentration im Rohwasser von 90 bis I $50 \mathrm{mg} / \mathrm{l}$. 
5. Hochbelastete Belebtschlammanlagen lassen sich ohne jeden künstlichen Zusatz mit Sicherheit und einwandfreiem Ergebnis betreiben.

6. Auf Grund unserer Versuche kommen wir zur Auffassung, dass bei Anwendung des Tropfkörperverfahrens mit einmaligem Abwasserdurchgang nur geringe Raumbelastungen $\left(R_{b}<0,8 \mathrm{kgBsb} / \mathrm{m}^{3}\right.$. Tag $)$ angewendet werden können, damit Abflüsse erzielt werden, die den Interessen des Gewässerschutzes genügen (auch wenn die Tropfkörper Höhen bis $\mathrm{zu} 3,5 \mathrm{~m}$ aufweisen). Die künstliche Belüftung übt auf die Abflussqualität keinen erkennbaren Einfluss aus und ist auch bei hochgeschichteter Körperfüllung betriebstechnisch nicht notwendig. Die Überdeckung der Tropfkörper gestattet allerdings, eine eventuelle Fliegenplage sicher zu verhindern, doch ist dies auch mittels geeigneter Betriebsweise bei offenen Körpern weitgehend möglich.

7. Setzt man eine gleichartige Qualität des Abflusses bezüglich $B s b_{5}$ bzw. Gehalt an organischem und Gesamtstickstoff voraus, so erweist sich der untersuchte, künstlich belüftete Tropfkörper bezüglich Belastungsfähigkeit mit Abwasser und Schmutzstoffen dem Belebtschlammverfahren als stark unterlegen (Abb. $\mathrm{I}_{4}$ und $\mathrm{I} 7$ ).

8. Bezüglich der Zusammenhänge zwischen den Reinigungsleistungen unserer Versuchsanlagen, der Qualität der erzielten Abflüsse und der Auswirkung der gereinigten Abwässer in fliessenden Vorflutern verweisen wir im übrigen auf die früheren Arbeiten aus unserem Institute (WUHRMANN, I95I $a, b$ ).

\section{LITERATURVERZEICHNIS}

BabBit, H. E., Sewerage and Sewpage Treatment (J. Wiley, New York 1947).

Barker, A. N., J. Proc. Inst. Sew. Purif. 1949, 7.

BLUNK, H., Gesundheitsing. 62, 323, 338 (1939).

Buswell, A. M., Shive, R. A., und Neave, S. L., Water Survey, Bull. Nr. 25 (I928).

Cameron, W. M., und Jamieson, A. R., J. Proc. Inst. Sew. Purif. 1950, 4 I7.

Chase, E. S., S. W. J.I) 16, 878 (1944).

Dekema, C.J., und Murray, K. A., Publ. Health (South Africa) 6, Nrn. 2 und 3 (I942).

Dekema, C. J., und Krige, P. R., J. Proc. Inst. Sew. Purif. 1949, 9 I.

ELY, H. M., Calif. Sew. Works J. 10, I7 (1938).

Fair, G. M., und Thomas, H. A., J. Proc. Inst. Sew. Purif, 1950, 235.

Gould, R. H., Municipal Sanit. 10, 185 (1939); S. W. J. 14, 70 (1942).

GREELEY, S. A., S. W. J. 15, TO62 (I743).

Haseltine, T. R., S. W. J. 10, Ior7 (1938).

1) S.W. J. = Sewage Works Journal. 
Heukelekian, H., Sew. Ind. Wastes 23, 945 (195I).

Hunter, A., und Cockburn, T., J. Proc. Inst. Sew. Purif. 1944, I2.

Husmann, W., und Lanz, T., Gesundheitsing. 61, 23 I (1938).

IMHOFF, K., Schweiz. Bauztg. 67, Nr. 18 (1949); Taschenbucb der Stadtentwässerung, 14. Aufl. (Oldenbourg, München 195I).

JAEGERS, K., und NIEMITZ, W., Gesundheitsing. 72, 83 (I95I).

JENkINS, S. H., und LocketT, W. T., Nature 151, 306 (I943).

JUNG, H., Gesundheitsing. 69, 306 (1948).

KESSENER, H. J., Rijksinstitut voor Zuivering van Afvalwater, Jaarverslag 1932, S. 52.

KROPF, A., Strasse und Verkehr Nr. 7 (I940).

LANZ, T. E., Dissertation ETH. (Zürich I937).

MülLER, J., Sonderdruck Schweiz. Z. Strassenwesen (Solothurn I929).

MülleR-NeuHaUS, G., Wasserwirtschaft 43, 7 (I952).

NESMEJANOFF, Gesundheitsing. 58, 47I (1935).

OKUN, D. A., S. W. J. 21, 763 (I949).

Pasveer, J. A., Commissie Afvalwaterzuivering T.N.O. Mitt. Nr. I (I949); Mitt.

Nr. 2 (I950); Mitt. Nr. 8 (I95I).

PIPE, F. E., und FARMER, J. E., Surveyor 91, 437 (1937).

Pönninger, R., Beiheft $\mathrm{I} 8 \mathrm{zu}$ : Gesundheitsing. (1938).

Ridenour, G. M., und Henderson, C. N., S. W. J. 8, 766, 924(1936); 9, 4I (1937).

ROHDE, H., Städtehygiene 1951, Nr. 9; Wasserwirtschaft 42, I95 (1952).

Ruchioft, C. C., und Moore, A., Publ. Health. Rep. 55, 393 (I940).

SAWYER, C. N., und STARR, N. M., S. W. J. 11, SI (I939).

SAWYER, C. N., und Rohlich, G. A., S. W. J. 11, 946 (I939).

SAWYER, C. N., S. W. J. 16, 925 (1944).

SETter, L. R., und Edwards, G. P., S. W. J. 15, 629 (I943); 16, 278 (1944).

SetTer, L. R., Carpentier, W. T., und Winslow, G. C., S. W. J. 17, 669 (1945).

SIERP, F, in: Die Stadtentwässerung in Deutscbland (G. Fischer, Jena 1934).

Sudgen, B., und Lloyd, L. L., J. Proc. Inst. Sew. Purif. 1950, I6.

VAN SLYKE, D. D., und FolCh, J., J. Biol. Chem. 136, 509 (1940).

van Slyke, D. D., McFadyen, D. A., und Hamilton, P. B., J. Biol. Chem. 150, $23 \mathrm{I}$ (I943).

SMITH, D. B., Sew. Ind. Wastes 24, 1077 (1950).

Standard Metbods for the Examination of Water and Semage (Am. Publ. Health Ass., New York 1946).

STANLEY, W. E., S. W. J. 21, 62.5 (1949).

Tomlinson, T. G., J. Proc. Inst. Sew. Purif. 1945, I40; $1950,338$.

UllRich, A. H., und SMiTH, M. W., Sew. Ind. Wastes 23, I248 (I95I).

VIEHL, K., Z. Hyg., Inf.-Krankh. 122, 533 (I940); Gesundheitsing. 64, 369 (I94I).

Vosloo, P. B. B., und O'ReIlly, T. P., J. Proc Inst. Sew. Purif. 1945, 160.

WHITEHEAD, H. C., Surveyor 68, 449 (I925).

WuhrmanN, K., VSA. Verbandsber. Nr. I4/2 (1949); Gesundheitsing. 72, 253,

(195I a); Strasse und Verkehr 37 (I95I b); Verh. Int. Ver. theor. angew. Limnol. $10,580(1949)$.

ZEHENDER, F., Schweiz. Z. Hydrol. 10, 4 (1948); Verh. Int. Ver. theor. angew. Limnol. 10, 597 (1949). 